\title{
Mechanics of Intermediate Filaments
}

\author{
Dissertation \\ for the award of the degree \\ "Doctor rerum naturalium" \\ of the Georg-August-Universität Göttingen
}

within the doctoral program

Göttingen Graduate School for Neurosciences, Biophysics, and Molecular Biosciences (GGNB) of the Georg-August University School of Science (GAUSS)

submitted by

\section{Bernd Nöding}

from Homberg (Efze)

Göttingen 2014 


\section{Thesis committee:}

Prof. Dr. Sarah Köster

Institute for X-Ray Physics

Georg-August-University Göttingen

Prof. Dr. Dr. Detlev Schild

Department of Neurophysiology \& Cellular Biophysics

Georg-August-University Göttingen

Dr. Thomas Burg

Department for Micro- and Nanotechnology

Max Planck Institute for Biophysical Chemistry, Göttingen 


\section{Members of the examination board:}

Reviewer: Prof. Dr. Sarah Köster

Institute for X-Ray Physics

Georg-August-University Göttingen

Second reviewer: Prof. Dr. Dr. Detlev Schild

Department of Neurophysiology \& Cellular Biophysics

Georg-August-University Göttingen

\section{Further members of the examination board:}

Dr. Thomas Burg

Department for Micro- and Nanotechnology

Max Planck Institute for Biophysical Chemistry, Göttingen

Prof. Dr. Helmut Grubmüller

Department for Theoretical and Computational Biophysics

Max-Planck-Institute for Biophysical Chemistry, Göttingen

Prof. Dr. Andreas Janshoff

Institute for Physical Chemistry

Georg-August-University Göttingen

Dr. Florian Rehfeldt

Third Institute of Physics - Biophysics

Georg-August-University Göttingen

\section{Date of oral examination:}

06.03.2014 


\section{Contents}

\begin{tabular}{lll}
\hline & Introduction & 1
\end{tabular}

2 State of the art 5

2.1 Assembly of intermediate filaments . . . . . . . . . . . . . 6

2.2 Dynamic changes in mature filaments . . . . . . . . . . . . . 8

2.3 Mechanical properties of bio-polymers . . . . . . . . . . . . . 8

2.4 Microfluidics . . . . . . . . . . . . . . . . . . . . . . . . . . . . 11

2.4 .1 Diffusion chamber devices . . . . . . . . . . . . . . 13

2.4 .2 Microchannel devices . . . . . . . . . . . . . . . . . . . . . . 14

\begin{tabular}{|lll}
\hline 3 & Materials and methods & 17
\end{tabular}

3.1 Vimentin . . . . . . . . . . . . . . . . . . . . . . . . . . . . . . 17

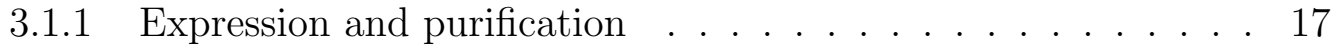

3.1 .2 Determining vimentin concentration . . . . . . . . . . . . . 18

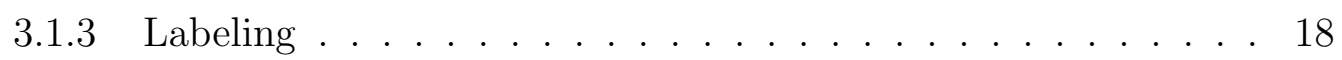

3.1.4 Reconstitution and assembly . . . . . . . . . . . . . . . . . . 20

3.1.5 Subunit exchange experiments . . . . . . . . . . . . . . . . . . 21

3.2 Soft lithography . . . . . . . . . . . . . . . . . . . . . . . . . . 22

3.2 .1 Photolithography . . . . . . . . . . . . . . . . . . 23

3.2 .2 PDMS device . . . . . . . . . . . . . . . . . . . . . . . . 24

3.3 Microfluidic devices . . . . . . . . . . . . . . . . . . . . . . . 25

3.3 .1 Microchannels . . . . . . . . . . . . . . . . . 26

3.3 .2 Diffusion chambers . . . . . . . . . . . . . . . . . . 27

3.4 Surface treatment of microfluidic devices $\ldots \ldots \ldots$. . . . . . . . . . . . . . 29

3.4 .1 Bovine Serum Albumin . . . . . . . . . . . . . . . . . . 30

3.4 .2 PLL-g-PEG . . . . . . . . . . . . . . . . . . . . . . . . 30

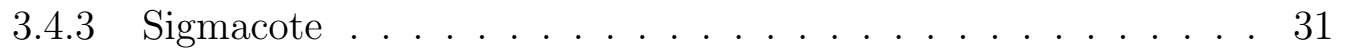

3.4 .4 Lipid bilayer. . . . . . . . . . . . . . . . . . . . 31 
3.4 .5 Lipid vesicle preparation . . . . . . . . . . . . . . . . . 32

3.5 Microscopy $\ldots \ldots \ldots$. . . . . . . . . . . . . . . . . . . . . . . . . . . 33

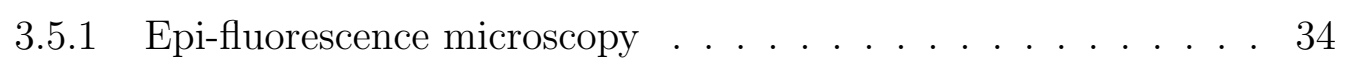

3.5 .2 Confocal microscopy $\ldots \ldots \ldots \ldots$. . . . . . . . . . . . 36

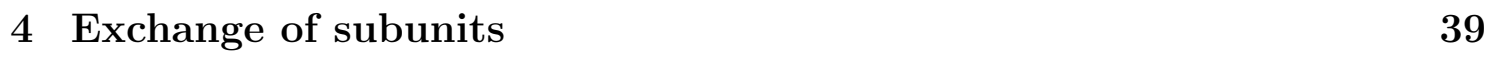

$4.1 \quad$ Initial considerations and experimnets . . . . . . . . . . . . . 39

4.1 .1 Microscopy methods . . . . . . . . . . . . . . . . . . . . 39

4.1 .2 Control experiments . . . . . . . . . . . . . . . . 41

4.1 .3 Filament polymorphism . . . . . . . . . . . . . . . 42

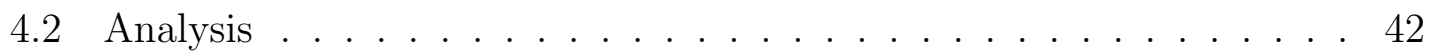

$4.2 .1 \quad$ Percentage of exchanged subunits with red filaments as reference 43

4.2.2 Fluorescence intensity of subunits relative to red background . 44

4.2 .3 Statistics . . . . . . . . . . . . . . . . . . . . . . . . . . . . . . 44

4.3 Results . . . . . . . . . . . . . . . . . . . . . . . . . . . . . . . . . . 45

4.3 .1 Comparison of analysis methods . . . . . . . . . . . . . 46

4.3 .2 Measurements with high sampling frequency . . . . . . . . . . 46

4.3 .3 Effects of different assembly methods and temperature . . . . 46

4.3 .4 End-to-end annealing . . . . . . . . . . . . . . . . . . 49

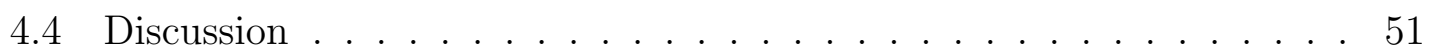

4.4 .1 The first 60 minutes of an experiment . . . . . . . . . . . 51

4.4 .2 Linear exchange of subunits . . . . . . . . . . . . . 52

4.4 .3 Diffusion of subunits is relevant for the exchange reaction . . . 53

4.4 .4 Comparison with literature . . . . . . . . . . . . . . . 55

4.4 .5 Implications of subunit exchange . . . . . . . . . . . . . 56

4.4 .6 End-to-end annealing and filament stability . . . . . . . . . 57

$\begin{array}{lll}5 & \text { Diffusion chambers } & 59\end{array}$

5.1 Characterization of the device . . . . . . . . . . . . . . . . . . . . 59

5.2 Vimentin assembly and disassembly . . . . . . . . . . . . . . 65

5.2 .1 Diffusion of vimentin into the chambers . . . . . . . . . . . 66

5.2 .2 Assembly of vimentin . . . . . . . . . . . . . . . . . . . . 67

5.2 .3 Disassembly of vimentin . . . . . . . . . . . . . 71

5.3 Discussion . . . . . . . . . . . . . . . . . . . . . . . . . . 75

5.3 .1 Inflow of vimentin and characteristic timescales . . . . . . 75

5.3 .2 Assembly of vimentin and subunit exchange . . . . . . . . . 76

5.3 .3 Disassembly of vimentin . . . . . . . . . . . . . . . 77

5.3 .4 The diffusion chamber device as a whole . . . . . . . . . . . . 78 
$\begin{array}{lll}6 & \text { Filaments in a confining geometry } & \mathbf{7 9}\end{array}$

6.1 Polymer physics . . . . . . . . . . . . . . . . . . . . . . . . . . . . . . . . 79

6.1 .1 The worm-like chain . . . . . . . . . . . . . . . 80

6.1 .2 The confined worm-like chain . . . . . . . . . . . . . . . 81

6.1 .3 Odijk's scaling law . . . . . . . . . . . . . . . 83

6.2 Analysis . . . . . . . . . . . . . . . . . . . 84

6.2 .1 Statistical considerations . . . . . . . . . . . . . . . 85

6.3 Results . . . . . . . . . . . . . . . . . . . . . . . . . . . . . . . 87

6.3 .1 Effects of changing channel width . . . . . . . . . . . . 88

$6.3 .2 \quad$ Effects of changing channel height . . . . . . . . . . . . . . . . 88

6.3 .3 Tangent-correlation fits . . . . . . . . . . . . . . . . . . 90

6.3 .4 Application to a scaling law . . . . . . . . . . . . . . . . 91

6.4 Discussion . . . . . . . . . . . . . . . . . . . . . . . . . . . . . . 93

6.4 .1 Persistence length of vimentin . . . . . . . . . . . . . . . 93

6.4 .2 Properties of confined filaments . . . . . . . . . . . . . . 97

6.4 .3 Reduction of configuration space . . . . . . . . . . . . . . . . 99

$\begin{array}{lll}7 & \text { Summary, conclusion and outlook } & 101\end{array}$

\begin{tabular}{ll}
\hline A Vimentin synthesis & 105
\end{tabular}

A.1 Expression in E.Coli . . . . . . . . . . . . . . . . . . . . . . . . . . 105

A.2 $\quad$ Purification of vimentin from bacteria pellet . . . . . . . . . . . . 106

A.2.1 Buffers . . . . . . . . . . . . . . . . 106

A.2.2 Centrifugation . . . . . . . . . . . . . . 106

A.2.3 Ion exchange chromatography . . . . . . . . . . . . . . . . . 108

\begin{tabular}{lr}
\hline B Abbreviations & 109
\end{tabular}

\begin{tabular}{ll}
\hline Bibliography & 113
\end{tabular}

\begin{tabular}{lr}
\hline List of publications & 125
\end{tabular}

\begin{tabular}{ll}
\hline Danksagung & 127
\end{tabular} 


\section{Chapter 1}

\section{Introduction}

Intermediate filaments are abundant in our body and provide key functions for the cell. Perhaps most importantly, from a physics point of view, intermediate filaments provide the cell with mechanical resilience. They complement the other main components of the cytoskeleton, actin filaments and microtubules, which play a critical role in cell motility and cellular transport. While both actin filaments and microtubules are well conserved in many species and, in any case, one organism, intermediate filaments occur in many different varieties. Alone in humans there are more than 70 distinctive intermediate filament proteins. Most of these are keratins, which are well known to occur in hair. All intermediate filaments share common characteristics in sequence as well as in structure. The name intermediate filament arises from the fact that the diameter of intermediate filaments $(9-11 \mathrm{~nm})$ lies between - or intermediate to - that of actin filaments $(7 \mathrm{~nm})$ and microtubules $(25 \mathrm{~nm})$. Figure 1.1 shows the three filament systems in a single cell line and can thus demonstrate the differences in not only the filaments themselves but also in their distribution and function in the cell. The use of different confining geometries for the cells allows the conclusion that the form of the vimentin network does not change due to subtle changes in cell geometry [1].

Naturally, mutations or simply the absence of intermediate filaments lead to often severe diseases. For example, Alexander disease, a progressive neurological degenerative disease, is a result of mutations in glial fibrillary acidic protein (GFAP) [2]. Epidermolysis bullosa simplex diseases, which are disorders in the skin and cause blistering, occur because of mutations in K5 or K14 keratins [3]. Mutations in vimentin are responsible for the formation of the autosomal dominant cataract, where the lens of the eye develops opacitiys early in life [4]. 
A
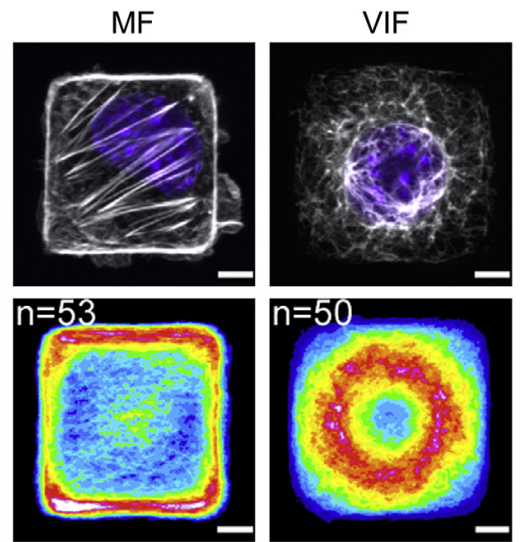

MT

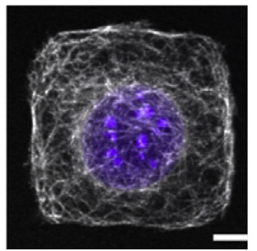

MT VIF
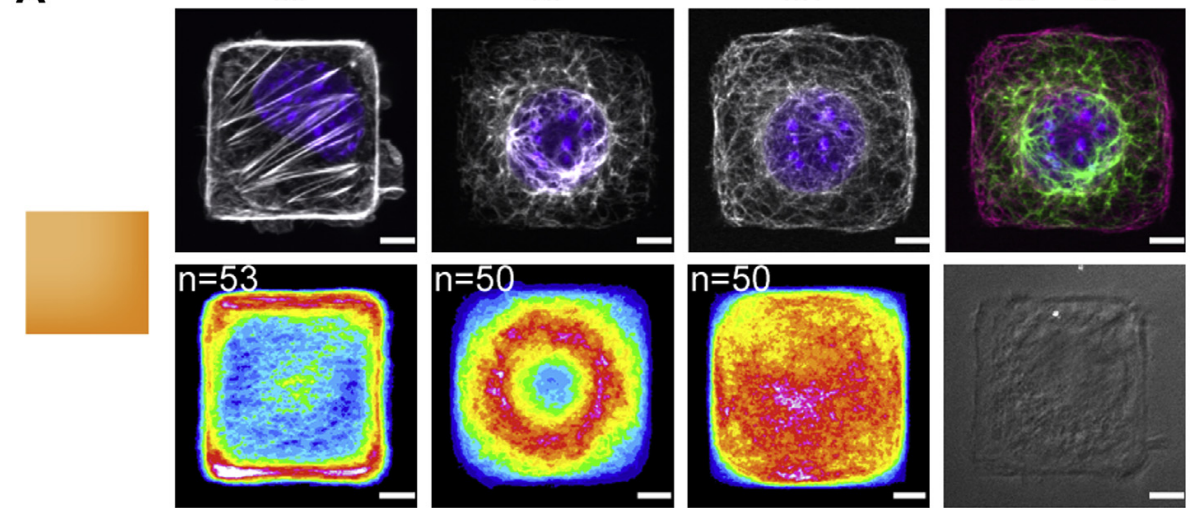

B

MF

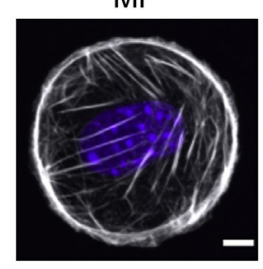

VIF

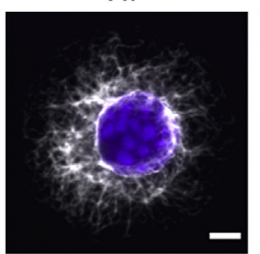

MT
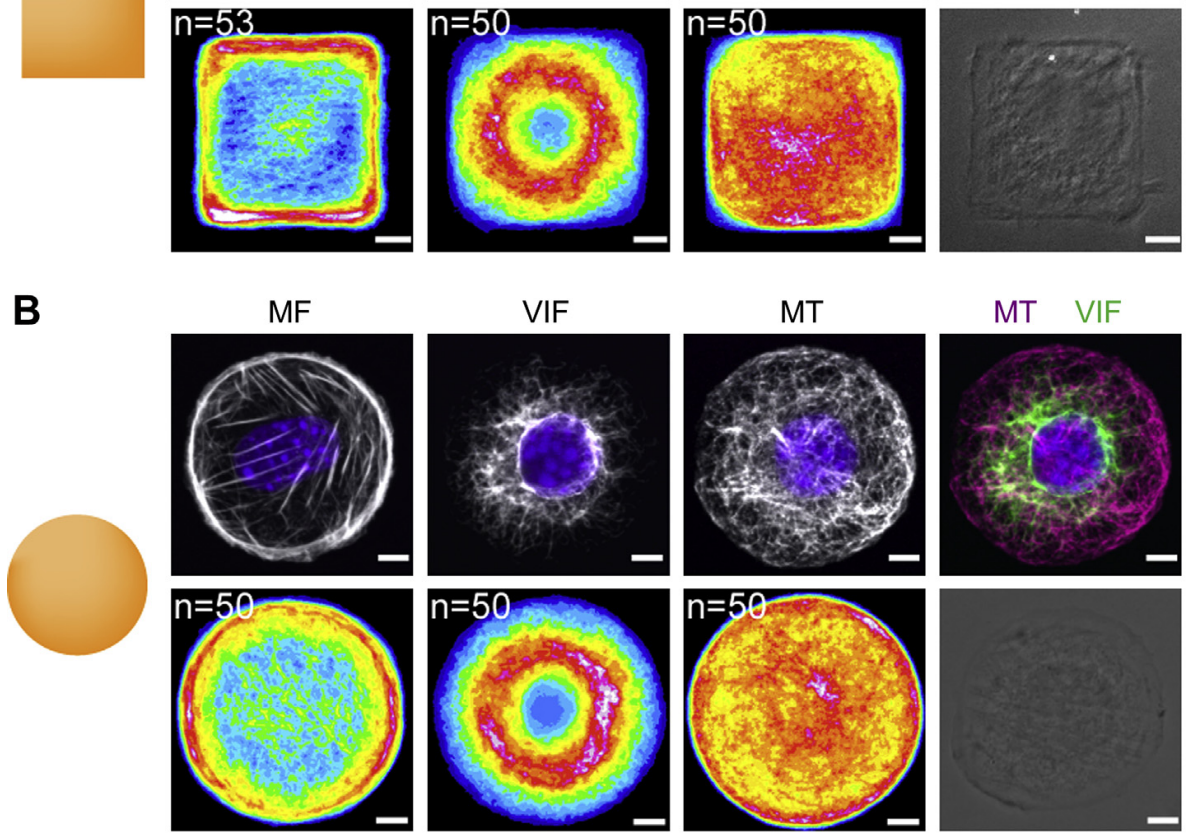

MT VIF

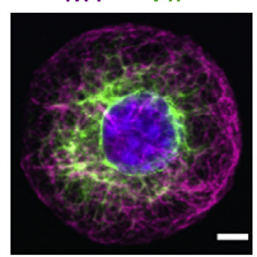

Low



High

Figure 1.1: Components of the cytoskeleton of mouse embryonic fibroblasts. Fibroblasts are observed on a patterned surface of either squares (A) or circles (B) with the same area. The distribution of the different cytoskeleton filaments is dependent on protein type. Actin filaments (MF) occur at higher frequencies near the cellular periphery. Vimentin filaments (VIF) mostly occur near the nucleus. Finally, microtubules (MT) are homogeneously distributed throughout the cell. The heat maps show an average of $n$ cells for the respective filament type. Scale bar $5 \mu \mathrm{m}$. Image with permission from [1]

In this thesis we will focus on the intermediate filament protein vimentin, which is abundant in mesenchymal cells and thus one of the most common intermediate filaments in our body.

In the cell, the intermediate filament network is defined by a number of characteristic properties. The assembly process and dynamical change of the individual filament are major attributes that, in the end, define this complex structure. We aim to further our understanding of the assembly and disassembly process. Related to these experiments, we also investigate the dynamic exchange of subunits in the mature filament. Apart from these studies, we know that the filaments feature certain mechanical properties which are necessary to fulfill their role in the cytoskeleton. In 
particular, we investigate the persistence length of the filaments. With results from these experiments, we are able to evaluate a scaling law in detail. All experiments mentioned thus far are performed in vitro on fluorescently labeled vimentin protein. Microfluidic techniques are essential for some of these experiments and are used in different forms.

In this thesis, we give an overview of the current knowledge of intermediate filaments in general and vimentin in particular as well as microfluidic techniques in chapter 2. In chapter 3 we explain the methods that are relevant for the experiments performed in the scope of this thesis. We show that intermediate filaments are dynamic structures and exchange subunits in chapter 4 . Chapter 5 focuses on our experiments on the assembly and disassembly process of vimentin filaments in socalled diffusion chambers. The conclusion to our experiments is the observation of vimentin filaments in confining microchannels as described in chapter 6. We present a summary of our findings on these topics and formulate a conclusion as well as possibilities for further research in chapter 7 . 


\section{Chapter 2}

\section{State of the art}

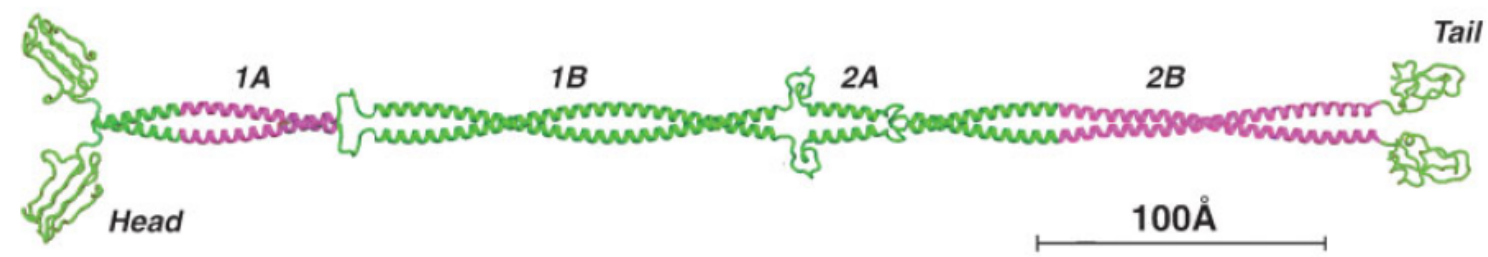

Figure 2.1: A vimentin dimer. Two vimentin monomers form a coiled-coil homodimer. The head region is thought to be relevant for later assembly stages. The tail region seems to play a less prominent role in the filament assembly process. Whereas the enclosed rod region is already relevant for the association into dimeric and tetrameric stages. Image with permission from [5]

Intermediate filaments occur in many different variants that share a characteristic secondary structure. These different variants can be categorized according to distinct properties. Up until now, intermediate filaments are assigned to one of six sequence homology groups. However, the number of these intermediate filament types has been changed in the past as new properties or filament types have been described. The intermediate filament types are the following: Types I and II are acidic and neutral/basic keratins, respectively and only assemble as heteropolymers. Here, a type I and a type II keratin form a heterodimer. Type III filaments include desmin, vimentin and GFAP. These proteins typically assemble into homopolymers, but may also form heteropolymers with other type III filaments. Type IV intermediate filaments are found mostly in nerve cells and include the different neurofilaments. The nuclear intermediate filaments, called lamins, are assigned to type V. Finally, type VI filaments include filaments found in the eye. The two proteins, phakinin and filensin are highly divergent from the other intermediate filament groups but are evolutionary strongly related to them and therefore still counted as such [6]. 
The characteristic appearance of all intermediate filament proteins is very similar. A rod like central structure, consisting of a long $\alpha$-helical region is enclosed by an amino-terminal head and a carboxy-terminal tail region. The head and tail region are more flexible than the rod region and also show more variance between different intermediate filaments than the rod. Figure 2.1 shows a dimer of the intermediate filament vimentin, which illustrates the position of the three main regions of the protein.

\subsection{Assembly of intermediate filaments}

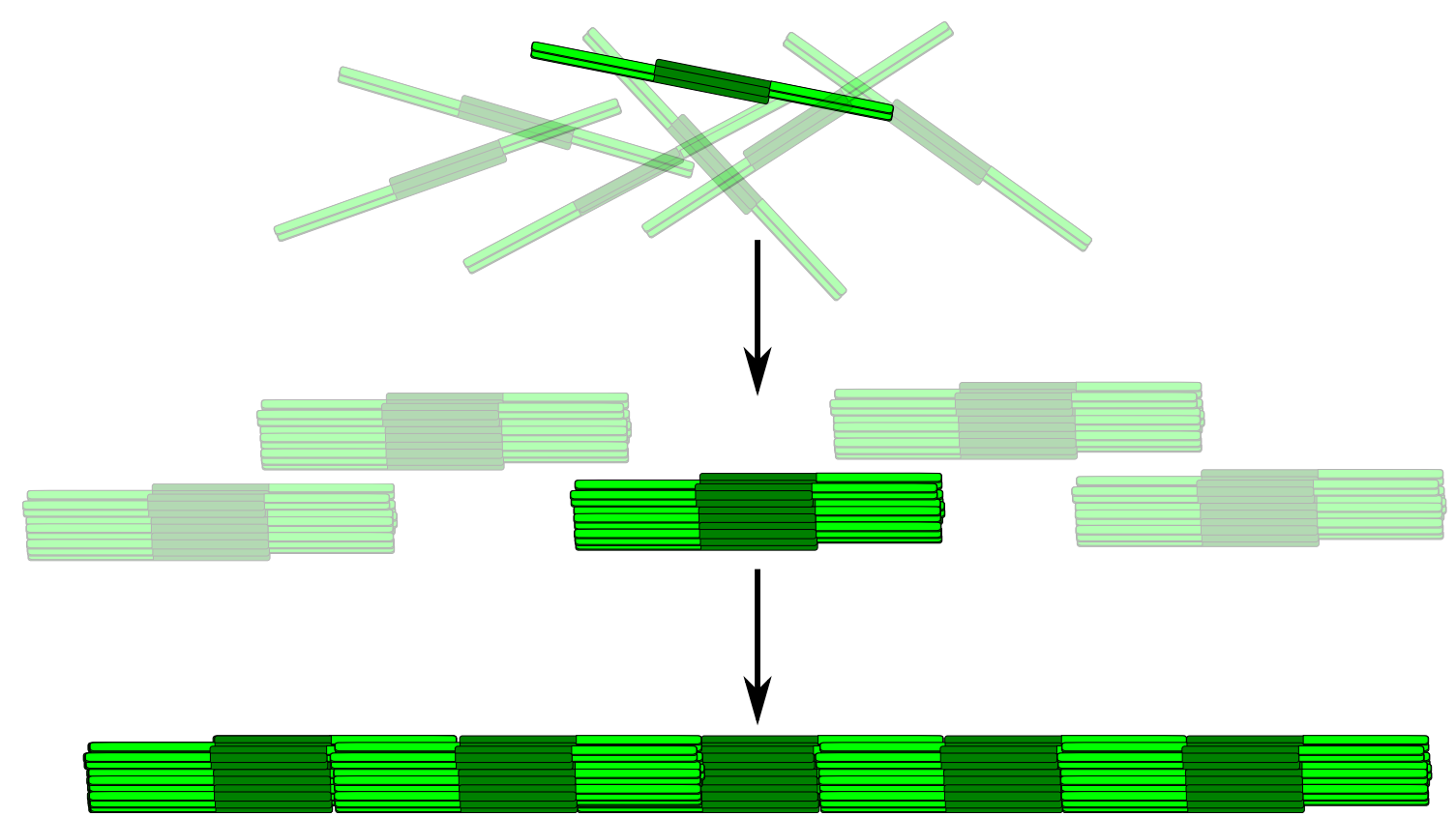

Figure 2.2: Different stages in the vimentin assembly process. In vitro an increase in salt concentration initiates the assembly process. First, tetramers align laterally to form a unit length filament with a total of 32 monomers. These unit length filaments then begin to form filaments by binding to the end of a filament which has a diameter of $17 \mathrm{~nm}$. Finally, the filament experiences a compaction step and reaches a final diameter of $11 \mathrm{~nm}$.

The assembly process differs from filament type to filament type. Type V filaments - lamins - are thought to assemble via the formation of long protofibriles which follow the dimeric state shown in figure 2.1 on the example of vimentin. However, the exact assembly mechanism is still unknown $[7,8$. A different assembly path is present in keratins, where heterodimers of type I and II proteins are formed, which then combine into a tetrameric state and begin to assemble into filaments by a more or less simultaneous lateral and longitudinal assembly processes [9]. Whether an 
intermediate step between lateral and longitudinal assembly exists, is unclear and hard to determine since keratin filaments assemble at high speeds. The assembly process for type III filaments, including vimentin is, in contrast, a hierarchical process, as shown in figure 2.2 [7]. Two homodimers bind together to form a tetramer. Tetrameric vimentin is the smallest vimentin assembly state that is observed in $2 \mathrm{mM}$ phosphate buffer. In vitro an increase in ionic strength results in the beginning of the actual assembly process into filaments. As a first step in the hierarchical process, 8 tetramers bind laterally and form a so-called unit length filament. These, unit length filaments join end-to-end and thus elongate into filaments 9 15]. At this point, the diameter of the filament is found to be $17 \mathrm{~nm}$. After a compaction step, the filament reaches a final diameter of $11 \mathrm{~nm}$. Recently, it has been determined that apart from elongation by addition of single unit length filaments, two filaments can form a single, long filament by end-to-end annealing [16, 17]. It is important to note that vimentin filaments can show polymorphism of the filament diameter. The cross-section of the filament must not always consists of 32 monomers ( 8 tetramers). Depending on the buffer conditions the cross-section of a vimentin filament can increase to as many as 84 monomers. Stable filaments with at least 44 monomers per cross-section are documented $[9,12$. The polymorphism of the filament can be influenced by using different assembly methods as described in chapter 3.1.4 12. Assembly in a dialysis bath will typically yield more uniform filaments than an assembly process started by dilution into assembly conditions [12. Herrmann et al. propose that the rapid dilution into assembly buffer leads to unit length filaments with a varying number of monomers which then form filaments exhibiting strong polymorphism. In contrast, the slower increase in ionic strength in a dialysis bath is thought to lead to a much more homogeneous unit length filament population 9 . The polymorphism varies strongly between individual intermediate filament species. Herrmann et al. propose that this finding points to a physiological relevance of the filament cross-section polymorphism [9].

The molecular binding processes governing the assembly process can in short be described as follows: Both tetramer and unit length filament formation depend heavily on the structure of the head domain. Head-truncated proteins often show altered assembly properties. Depending on the mutation of the protein, the assembly process may proceed up to a certain point or fail altogether. Through variations in the vimentin amino acid sequence, it is possible to determine the amino acids which are critical for the assembly process. Thus it is possible to infer likely assembly 
mechanisms. It is noteworthy that even very small alterations to the sequence of vimentin lead to strong changes in the assembly mechanism. For instance, the change of a single amino acid can hinder the protein from forming standard filaments [18]. A proposed mechanism [7, 19 for the tetramer and unit length filament assembly is the interaction of the arginine amino acids in the head region with the acidic rod of the coiled-coil dimer.

\subsection{Dynamic changes in mature filaments}

Mature intermediate filaments - that is, fully assembled intermediate filaments after the compaction step - are not necessarily unable to interact with other intermediate filament aggregates. This means that an exchange of some kind of subunit from one filament into a different or the same filament can occur. Speculation on this topic has started more than twenty years ago [20]. Early indications for the exchange of subunits in intermediate filaments have been obtained by fluorescence recovery after photobleaching (FRAP) experiments, but have been ambiguous [21]. Later studies on living cells with intermediate filaments labeled with two different fluorescent proteins show that an exchange of subunits is indeed taking place in the living cell for vimentin and neurofilaments [17]. However, it remains unclear to what extent this subunit exchange is governed by cellular mechanisms or if the exchange of subunits is only dependent on the properties of the intermediate filament itself.

Further, one might ask, if mature filaments can break in the middle and reform by end-to-end annealing in an equilibrium reaction. This was recently confirmed experimentally for neurofilaments in neurons [22]. In these experiments, long filaments labeled with two different fluorescent dyes were combined in a single cell. Given enough time, the long filaments broke down and reassembled into new filaments, resulting in filaments with short stripes in each color instead of two single, long parts. As above, it is unclear whether the filament splitting events are an inherent property of the filaments or if a cellular regulation mechanism is responsible.

\subsection{Mechanical properties of bio-polymers}

Figure 2.3 shows both intermediate filaments and actin filaments in a cell. In this case, the networks of both desmin and vimentin intermediate filaments are similar in appearance. However, in general the structure of the network can vary strongly 


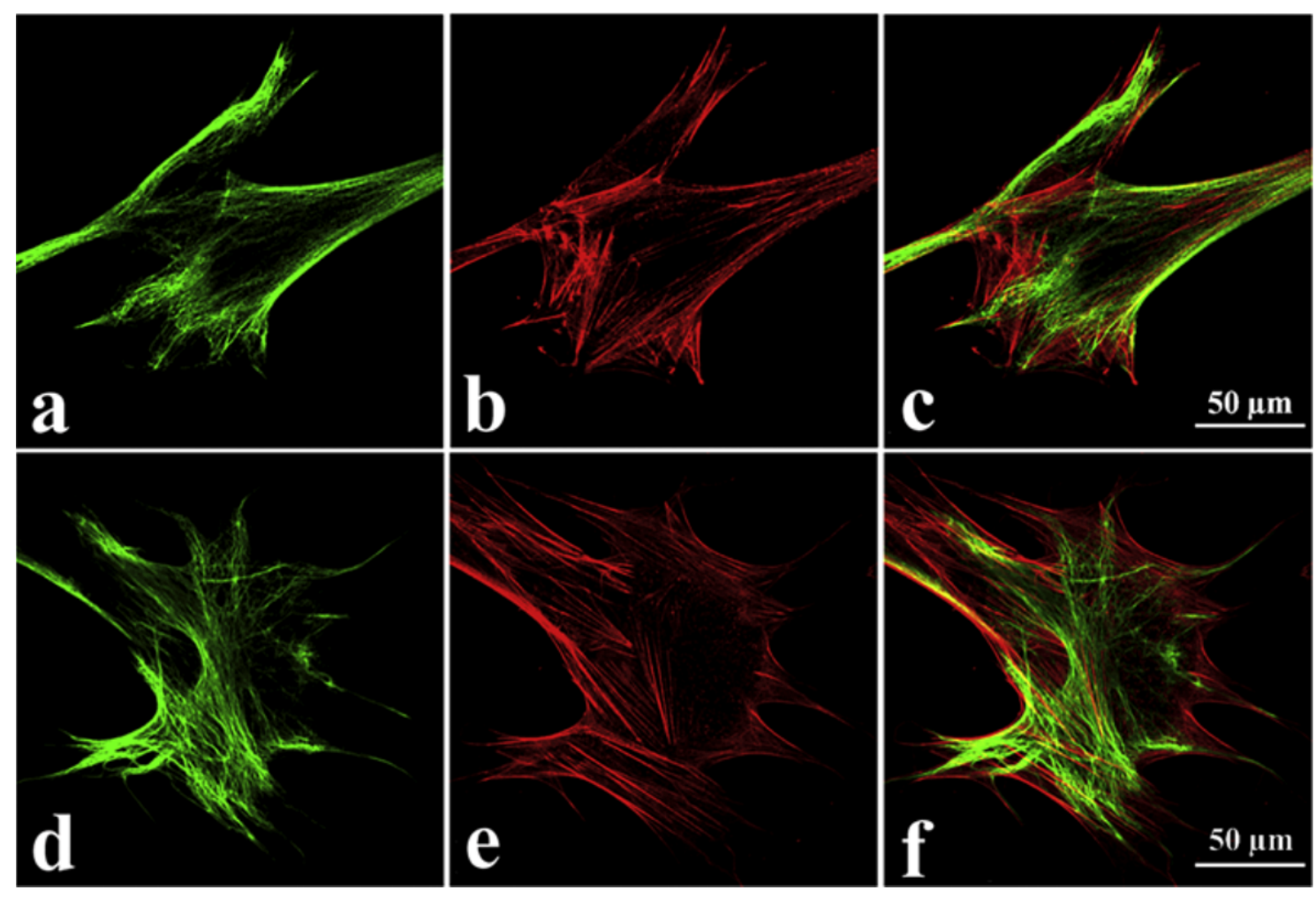

Figure 2.3: Stained cytoskeleton of human hepatic stellate cells. a) desmin, d) vimentin, (b,e) $\alpha$-smooth muscle actin, c) merge of $\mathbf{a}), \mathbf{b}$ ) and $\mathbf{f}$ ) merge of $\mathbf{d}$ ),e). Image with permission from 23 .

between different cell types with their specific intermediate filaments 2428 . This variance in the intermediate filament system is thought to have implications for the mechanical properties of the different cell types. However, the intermediate filament system is not the only component of the cell that has an influence on the mechanical properties of the cell. Thus, it is not wholly clear if the differences in the intermediate filament network morphology in different tissue types exist because of the specifics of the intermediate filament or if these differences are a results of the different purposes of the tissues. Of course, a strict separation of both issues is hardly possible. In any case, if we want to truly understand these complex structures, we first have to investigate and understand the properties of the individual filaments. The mechanical properties of a filament are represented by different measures. First, one may consider the stretching of the polymer, which is characterized by the tensile strength. Intermediate filaments do indeed stretch under stress as discussed in references [29,30]. The filaments exhibit a strong non-linear answer to stress. For low strain, the filament readily extends. However, with increasing strain the filament becomes much stiffer. This behavior is thought to be the result of the hierarchical 
make up of the filaments where, depending on the applied strain, different internal structures respond to the strain by deformation. Thus, intermediate filaments have been found to be very stretchable, an elongation of more than a factor of 2 has been observed under strain [31]. The elongation goes hand in hand with a decrease in diameter.

Apart from the behavior under strain, the bending stiffness $\kappa$ of a polymer is of great interest. In the context of biophysics, this value is usually written in terms of $k_{b} T$ and is called the persistence length

$$
L_{P}=\frac{\kappa}{k_{b} T} .
$$

In practice, there are mainly two different methods to determine this value: The use of rheology tools on bulk samples or the analysis of individual filament contours. Rheology measurements can again be roughly split into two groups, microrheology and the classical macroscopical rheology. For the microrheology measurement many particles are inserted into the sample. The particles can then be observed via optical microscopy or some other tool such as a light scattering setup. The material properties can then be obtained by evaluating the Brownian motion of the particle in the sample or alternately by actively moving the particle with optical tweezers or a magnetic field and monitoring its response. In a macro-rheology experiment the filament solution is placed between two plates or cones, which are then rotated. The linear viscoelastic shear modulus

$$
G(\omega)=G^{\prime}(\omega)+i G^{\prime \prime}(\omega)
$$

is observed for different frequencies $\omega$. The persistence length can in turn be calculated from these observations as described for example in reference [32]. In the case of vimentin, Schopferer et al. find a persistence length of $L_{P}=415 \pm 45 \mathrm{~nm}$ with macro-rheology methods.

Measurements on individual vimentin filaments adhered to different surfaces yield values for the apparent persistence length from about 300 to $1000 \mathrm{~nm}$ [33]. The adhered and fixated filaments are imaged with an atomic force microscope, which yields images with resolutions of about $30 \mathrm{~nm}$. However, Mücke et al. note that the interaction of the filaments with different surfaces leads to different apparent persistence lengths. With these interactions in mind, Mücke et al. conclude that vimentin filaments have a persistence length of about $1 \mu \mathrm{m}$.

The values for $L_{P}$ found for other intermediate filaments are in the same range as those found for vimentin filaments. Measurements with different techniques 
generally yield values of $0.3 \mu \mathrm{m} \lesssim L_{P} \lesssim 1.3 \mu \mathrm{m}$ for different filament types [32 35]. This is not very surprising given the generally very similar makeup of the filaments.

When we compare the stiffness of intermediate filaments with other bio-filaments we find that actin filaments have a persistence length in the range of $\approx 15 \mu \mathrm{m}$ [36 38 and microtubules have a persistence length of about $1 \mathrm{~mm}$ [37]. Interestingly, the persistence length for intermediate filaments is lower than those of the other two filament types despite having a larger cross-section than actin filaments. Microtubules have a larger diameter than intermediate filaments and posses a vastly different structure. However, solely based on a size comparison, the differences in persistence length between microtubules and intermediate filaments are still enormous. When we now instead focus on the stretching of the filaments, we find that the different polymers show distinctive behaviors. While both actin filaments and microtubules rupture before they elongate significantly [39,40], intermediate filaments elongate strongly before breaking.

\subsection{Microfluidics}

Microfluidic methods find applications in, for example, physics, chemistry and biology in various forms. The "micro" in microfluidics has several meanings for the properties of the actual microfluidic device. We handle small fluid volumes in small geometries. Effects, which only play a role on very small scales, may be central for such devices. For example, the dominance of the laminar flow regime and the strong impact of diffusive processes are often important factors [41]. Also, surface effects can have a great impact in microfluidic devices and can be employed in different manners [42]. The actual circumstances in the microfluidic device can often be described by dimensionless numbers, such as the Péclet number

$$
\mathrm{Pe}=\frac{U_{0} L_{0}}{D}
$$

which relates convection and diffusion or the Reynolds number, which gives the ratio of inertial and viscous forces

$$
\operatorname{Re}=\frac{\rho U_{0} L_{O}}{\eta}
$$

Here, $L_{0}$ and $U_{0}$ are characteristic length scales and velocity scales. $D$ is the diffusion coefficient, $\rho$ is the fluid density and $\eta$ the dynamic viscosity of the fluid.

The use of microfluidic devices has become a widespread in the last 10 years. This development is not very surprising, since microfluidics allows us to manipulate 
minute amounts of sample solutions in manifold ways. A common method to construct a microfluidic device is the use of PDMS, which is sealed with a glass cover slip as described in chapter 3.2. However, many different techniques have been developed that have unique advantages in certain areas. For example, devices can be made from silicon, glass and different plastics or rubber like polymers. Techniques for the fabrication of the microfluidic devices are as different as the materials they are made from and include photolithography, ion beam etching and molding techniques. A rather comprehensive review paper by Nge et al. gives a broad overview of possible material choices and their properties as well as a selection of applications 43 .

In their most essential form, microfluidic devices consist of nothing more than inlets, a basic channel geometry and outlets. Also, observation of the sample via optical microscopy or some other tool is generally a desirable feature. In addition to these essential techniques, manifold ways for the manipulation of the fluids and reactants in the device have been developed. For example, it is possible to construct valves in the devices, which can be used to precisely arrest flow or mix different components. Electric or magnetic fields can be established with high precision. They allow the experimentalist to discriminate between charged and uncharged or polar and apolar particles and thus construct a sorting mechanism. It is also possible to directly include optical measurement and manipulation equipment in the device and thus produce a so called lab-on-a-chip.

As described above, the possibilities for the use of microfluidic devices are manifold. However, they are especially useful, when only small quantities of an, often biological, sample are available. Also, the production of microfluidic devices with soft-lithography methods, as described in chapter 3.2, allows for changes in the device geometry with only moderate effort. Apart from considering the channel geometry, it is also important to make certain that the sample is compatible with the concept of the device. For instance, it is clear that the surface to volume ratio in a microfluidic device is inherently large when compared to a macroscopic experiment. Thus, it is necessary to make certain that the interaction of the sample with the surfaces of the microfluidic device only happens in desired ways. The desired behavior can be achieved by a considerate choice of device material or the use of various surface treatments. A selection of such treatments and their application is described in chapter 3.4. A broad overview [44,45] of established surface treatments can be found in the reviews by Wong and Ho or Makamba et al.. In addition to these considerations it might be necessary to adjust the typical length scales in the device to the diffusion properties of the sample to achieve a desired effect or time 
resolution. Here, the Péclet number can be used to assess the properties of the device.

After this brief introduction into microfluidics, we want to present the two device concepts that are most relevant in the scope of this thesis: the diffusion chamber device and the microchannel device.

\subsubsection{Diffusion chamber devices}

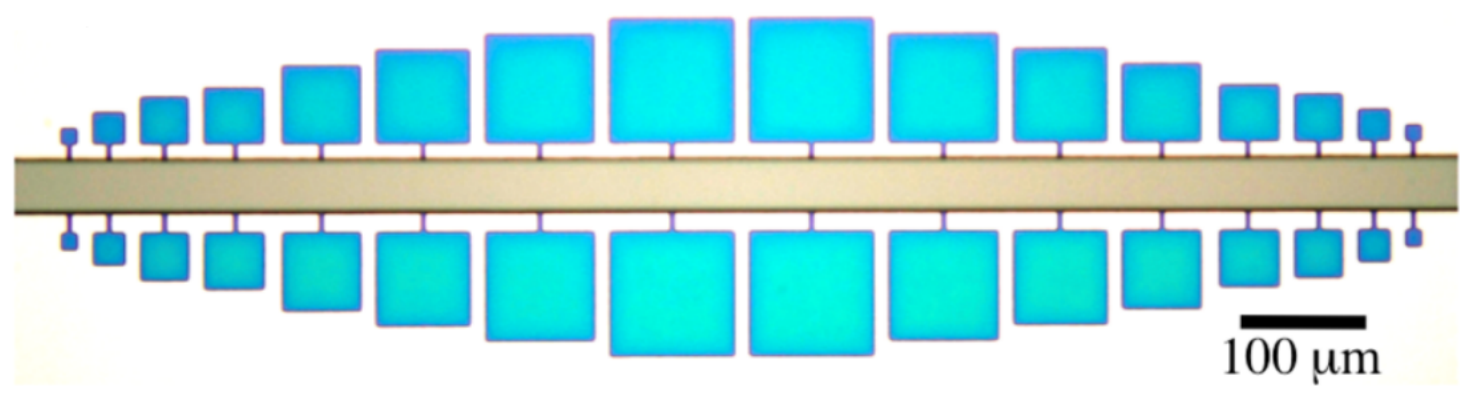

Figure 2.4: Diffusion chamber device. In this dual height diffusion chamber device, the actual chambers of low height are visible in blue. The main channel that supplies substances to the chambers by diffusion is visible in gray. With permission from [46].

Deshpande and Pfohl describe small reaction chambers in a microfluidic device shown in figure 2.4 [46]. These chambers are connected via small inlets to a main channel and can thus exchange salts or proteins by diffusion. The possible uses of this microfluidic design are manifold, as it can basically be employed as a micro-dialysis system. The chambers can be loaded with sample - Deshpande and Pfohl observe actin filaments - and the reaction of this sample to changes in the buffer composition can be observed without time delay normally encountered when reactions are carried out in reaction vessels. In this example, the sensitivity of the actin filaments to $\mathrm{Mg}^{2+}$ ions is tested. This is possible because of the differences in diffusive behavior. The low diffusion times for small ions and at the same time high diffusion times of the much larger proteins keep the actin filaments in the chamber, while salt ions can be exchanged. Additionally, it is possible to repeatedly change the buffer composition while keeping the chambers flow free. This can be useful to observe both directions of a reversible reaction with high time resolution. Basically most other experiments that could otherwise be realized with the help of dialysis tubing can in principle also be performed in these diffusion chamber devices with the added benefit of direct observation in situ. 
The diffusion chamber design has other advantages apart from the diffusive reactant exchange. For instance, the confining geometry can be used to mimic the properties of a cell. Deshpande and Pfohl use this to their advantage by observing the geometrical intricacies of actin networks in chambers of differing geometry.

With this premise, it is relatively straightforward to formulate experiments that can use the unique advantages of this microfluidic setup. Our adaption of this system and its uses with respect to the further exploration of the properties of vimentin filaments are described in detail in chapters 3.3 .2 and 5 , respectively.

\subsubsection{Microchannel devices}
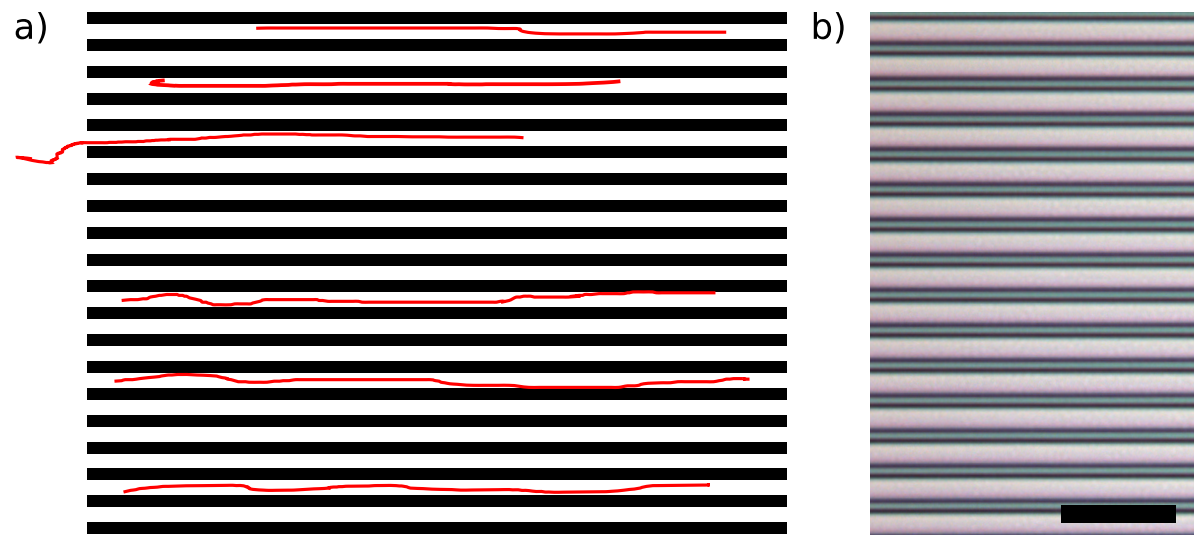

Figure 2.5: Microchannel device for the confinement of bio-polymers. a) Sktech of a microchannel device with confined filaments. Many channels are arranged parallel to each other for maximum redundancy. Filaments (drawn in red) are flushed into these channels and confined by a hard wall potential and therefore show a distinctive behavior. b) Microscopy image of a microchannel device. Channels are visible as dark lines, the PDMS the device is made from is represented by bright lines. Scale bar $20 \mu \mathrm{m}$

Microfluidic devices can not only be used to create reaction systems as detailed above, but can also be constructed to mimic biological systems. For instance, the cell is naturally rather densely packed with various functional units, bio-polymers and vesicles. The effect of this tight packing is a confinement that also has an influence on the different cellular polymer systems. As is often the case in physics, it is desirable to reduce the complexity of the experimental system, to fully understand it. Thus, the confining properties of the cell can be mimicked by producing microfluidic devices consisting of straight microchannels with a width of a few microns as shown in figure 2.5 . 
In reference 36] the behavior and analysis of fluorescently labeled actin filaments confined in such microchannels is described. A second, very popular polymer system is that of DNA strands. Many different experiments on confined DNA strands have been performed in the past [47 50]. All these approaches have in common that they investigate the mechanical properties of the confined polymer. This investigation can be extended to a more abstract consideration of the polymer properties that can be expressed through, for example, a scaling law. Of particular interest to us is a scaling law formulated by Odijk, which predicts the properties of a strongly confined polymer [51]. This scaling law has already been formulated in the 1980s, but a complete experimental validation has up until now been missing. There exist some other scaling laws describing polymers in a confining channel geometry. Apart from Odijks scaling law, a scaling law first described by de Gennes considers polymers under loose confinement. Scaling laws describing the regimes between that covered by Odijk and de Gennes have also been described in some detail [52, 53].

The measurement of the polymer properties with the help of a microchannel device has the advantage that only individual filaments are investigated and at the same time undesired interaction with surfaces is minimal. We use this to our advantage in experiments on confined vimentin intermediate filaments as described in chapters 3.3.1 and 6. There, we also take a detailed look at Odijks scaling law with respect to vimentin filaments. 


\section{Chapter 3}

\section{Materials and methods}

\subsection{Vimentin}

\subsubsection{Expression and purification}

For our experiments, we rely on purified protein samples, which for the purpose of labeling should have a concentration in the range of $1 \mathrm{~g} / \mathrm{L}$. To this end, we use vimentin protein expressed in transfected E.coli, which produce sufficient amounts of high quality protein. Protein synthesis is described in detail in appendix A. The protein is stored in a buffer of $8 \mathrm{M}$ Urea, $10 \mathrm{mM}$ DTT, $10 \mathrm{mM}$ TRIS, $5 \mathrm{mM}$ EDTA, $0.5 \mathrm{mM}$ EGTA and $10 \mathrm{mM} \mathrm{MAC}$ at $-80^{\circ} \mathrm{C}$.

The purified protein is tested with different methods to guarantee optimal quality. A SDS page gel is used to confirm that the purification product has a molecular mass of $53 \mathrm{kDa}$, as expected for vimentin. The amount of protein with molecular masses other than $53 \mathrm{kDa}$ should be very low, because these proteins would likely be fragmented vimentin parts or bacterial components not removed during the purification process. Furthermore, we observe the assembled filaments with high resolution imaging techniques such as electron microscopy or atomic force microscopy and look at the filament morphology in detail. A comparison with good quality filaments gives information on the sample quality, filament diameter should lie in a certain range and the filament contour should be smooth. Naturally, the final quality control step is the assembly of fluorescently labeled protein into filaments. 


\subsubsection{Determining vimentin concentration}

For an ideal labeling and assembly process, it is critical to work with a vimentin solution with a well defined concentration. To this end we use a NanoDrop ND-1000 spectrophotometer (NanoDrop Technologies Inc., Wilmington, USA), which can determine the protein concentration from a sample volume of $2 \mu \mathrm{L}$. In our case, we measure the light absorbance $A$ of the protein at a wavelength of $280 \mathrm{~nm}$ :

$$
A=\log \left(\frac{I}{I_{0}}\right)=\varepsilon l_{A} c
$$

Here, we use the extinction coefficient $\varepsilon=24.24 \cdot 10^{3} \mathrm{M}^{-1} \mathrm{~cm}^{-1}$ [54], the optical light path $l_{A}$ and the protein concentration $c$. The amino acids absorbing at $280 \mathrm{~nm}$ are mostly tyrosine and tryptophan, and consequently the absorbance $\varepsilon$ of a given protein can be estimated by counting the number of those amino acids in the protein sequence. The extinction coefficient obtained by this method is very similar to that found in [54], which we use to determine the protein concentration in our samples. Measurements on fluorescently labeled samples are corrected for the absorbance of the dyes at $280 \mathrm{~nm}$.

In addition to the measurement of the protein concentration itself, the NanoDrop also allows us to determine the amount of fluorescent dyes in the sample. We use this ability to define the ratio of labeled to unlabeled protein before the assembly into filaments is started.

\subsubsection{Labeling}

In the case of vimentin the maleimide labling chemistry is a very convenient choice, because only a single cysteine amino acid is found in the protein. In the wild type protein the cysteine is located at position 328 in the amino acid sequence. This corresponds to a location in the rod region of the protein. A label at this position is thought to interfere with the assembly process that entails close aligning in the rod regions. To solve this problem, the cysteine at position 328 is replaced by an alanin. In turn, a single cysteine is added to the C-terminus of the protein. With this modification, the maleimide label no longer attaches at the rod region but rather binds to the tail region of the protein. The interference of the label with the assembly process in this region should be far less pronounced or even absent.

The labeling protocol used by us is based on work from Winheim et. al. [16]. It can be used with a wide variety of dyes, as long as they are available with maleimide 


\begin{tabular}{l||lll} 
Dye & $\lambda_{e x}[\mathrm{~nm}]$ & $\lambda_{\text {em }}[\mathrm{nm}]$ & quantum yield \\
\hline \hline AlexaFluor 488 & 495 & 519 & 0.92 \\
AlexaFluor 532 & 532 & 554 & 0.61 \\
AlexaFluor 594 & 590 & 617 & 0.66 \\
Atto 647N & 644 & 669 & 0.65 \\
Dyomics DY-521XL & 523 & 668 & -
\end{tabular}

Table 3.1: The fluorescent dyes used in our experiments listed with the ideal wavelength of excitation $\lambda_{e x}$ and maximum emission $\lambda_{e m}$ as well as the quantum yield 55 58. The dyes mostly used by us are printed in bold.

chemistry. A list of dyes that we have used successfully to label vimentin can be found in table 3.1. For our experiments we mainly used AlexaFluor 488 and Atto $647 \mathrm{~N}$ dyes. AlexaFluor dyes are obtained from Invitrogen GmbH, Darmstadt, Germany, Atto dyes from ATTO-TEC GmbH, Siegen, Germany and Dyomics dyes from Dyomics GmbH, Jena, Germany.

Irrespective of the dye type, the protocol for the labeling process remains unchanged. As the labeling procedure works best when $1 \mathrm{~mL}$ of $1 \mathrm{~g} / \mathrm{L}$ protein solution is used, an appropriate amount of vimentin solution from storage at $-80^{\circ} \mathrm{C}$ is placed into dialysis tubing with a cut of at $50 \mathrm{kDa}$ (SpectraPor 7, Spectrum Europe B.V., Breda, Netherlands) and is dialysed in three steps into $20 \mathrm{mM}$ phosphate buffer $\mathrm{pH} 7$ [16, 59]. The first dialysis step is carried out in $1 \mathrm{~L}$ of a solution of $12 \mathrm{mM}$ phosphate buffer and $6 \mathrm{M}$ urea for 30 minutes. Two consecutive steps take place in $1 \mathrm{~L} 20 \mathrm{mM}$ phosphate buffer for 30 minutes each. If necessary, the vimentin concentration is adjusted to $1 \mathrm{~g} / \mathrm{L}$ after dialysis. The fluorescent dye is dissolved in water free DMSO [59] to obtain a $10 \mathrm{mM}$ stock solution, which can be stored at $-20^{\circ} \mathrm{C} .20 \mu \mathrm{L}$ of the dye stock solution are added to $1 \mathrm{~mL}$ of the vimentin solution in four portions of $5 \mu \mathrm{L}$. After each addition of dye, the solution is vortexed briefly and incubated for 5 minutes. After the total amount of dye is added to the solution, it is incubated over night.

To remove excess dye, which might also bind unspecifically to the vimentin protein, $100 \mu \mathrm{L}$ of $1 \mathrm{M}$ L-cystein (CarlRoth, Karlsruhe, Germany) $20 \mathrm{mM}$ phosphate buffer solution is added to the dye/vimentin solution and incubated for 1 hour. $1 \mathrm{~mL}$ dye/vimentin solution is applied on a PD MidiTrap-G25 desalting column (GE Healthcare Europe GmbH, Freibung, Geramny), which has been equilibrated 
with $20 \mathrm{mM}$ phosphate buffer. Two further columns are equilibrated with so called "storage solution" (9 M Urea, 10 mM DTT, 10 mM TRIS pH 7.5, 5 mM EDTA and $10 \mathrm{mM}$ PMSF) while the first column is eluated with $1.5 \mathrm{~mL}$ storage solution. The eluate from the first column, containing the labeled protein, is than placed on the second column for further purification. Because the output volume of the first column is larger than $1 \mathrm{~mL}$, we split the solution into two portions of $0.75 \mathrm{~mL}$. After this volume has entered the column bed, we add $0.25 \mathrm{~mL}$ of storage buffer. Now, both columns are eluated with storage buffer. The collected eluate contains fluorescently labeled vimentin and contains only very low amounts of free or unspecifically bound dye molecules. The fluorescently labeled vimentin is aliquoted and can be stored at $-80^{\circ} \mathrm{C}$ for several months.

Fluorescently labeled and unlabeled protein are mixed before the assembly into vimentin filaments is initiated. Typically we choose a percentage of $15 \%$ labeled protein, which ensures good assembly properties as well as good visibility in the experiments. To calculate the resulting ratio of labeled to unlabeled tetramers, we assume a random distribution of the labeled and unlabeled monomers in a tetramer. We do not consider influences on this distribution by specific interactions between labeled monomers. Under this assumption we obtain a fraction of $p_{0}=0.85^{4}=52 \%$ tetramers that are not fluorescently labeled. Therefore, only $0.5 \%$ of all unit length filaments are statistically label free, assuming a unit length filament consists of eight tetramers.

\subsubsection{Reconstitution and assembly}

After labeled and unlabeled vimentin are mixed at the required ratio, the total protein concentration is adjusted to $0.4 \mathrm{~g} / \mathrm{L}$. Now the protein is dialysed into $20 \mathrm{mM}$ phosphate buffer with $\mathrm{pH} 7.5$ in three steps of at least 30 minutes. The procedure is performed analogously to the dialysis steps prior to the labeling reaction. The thus reconstituted protein can be stored at $4^{\circ}$ for several days.

To initiate the assembly into vimentin filaments, protein solution with a concentration of $0.4 \mathrm{~g} / \mathrm{L}$ is mixed with an equal volume of $200 \mathrm{mM} \mathrm{KCl}$ (CarlRoth, Karlsruhe, Geramany) $20 \mathrm{mM}$ phosphate buffer solution in a reaction vessel. The assembly in a reaction vessel works best at $37^{\circ} \mathrm{C}$. After 12 to 24 hours the assembly process has proceeded sufficiently and filaments with lengths of up to $50 \mu \mathrm{m}$ have assembled.

As an alternative to the assembly in a reaction vessel, the assembly can also be 
initiated by adding $\mathrm{KCl}$ to the dialysis buffer. Here, we increase the $\mathrm{KCl}$ concentration in the dialysis buffer to $100 \mathrm{mM}$. However, it is important to adjust the protein concentration to $0.2 \mathrm{~g} / \mathrm{L}$ prior to the start of the assembly. Assembly times are identical to those in a reaction vessel, this also means that the temperature of the dialysis bath is adjusted to $37^{\circ} \mathrm{C}$. The assembly in dialysis tubing has the advantage that free dye molecules and other small debris can leave the dialysis tubing during the assembly process. Interestingly, the filament mass per length is more uniform when filaments are assembled in dialysis tubing [12]. Therefore, the assembly in dialysis tubing is the preferable method in many cases.

\subsubsection{Subunit exchange experiments}

For experiments on the exchange of subunits we use both filaments assembled in a reaction vessel and in dialysis tubing. We begin by dialysis in $2 \mathrm{mM}$ phosphate buffer. We use tetrameric vimentin labeled with AlexaFluor 488 ("green") and ATTO 647N ("red"), respectively. Dialysis is performed strictly separate for both dyes.

After dialysis concentrations are adjusted according to the assembly method. With procedures as described in chapter 3.1 .4 we obtain three differently assembled filaments.

- green filaments, assembled in a reaction vessel

- green filaments, assembled in a dialysis bath

- red filaments, assembled in a dialysis bath

These different filament types are then combined with tetrameric vimentin or with other filaments in order to observe an exchange of subunits. We use the following combinations:

- Combination I: green and red dialysed filaments. Both at a concentration of $0.1 \mathrm{~g} / \mathrm{L}$.

- Combination II: green dialysed filaments and tetrameric red vimentin. Concentrations for both are $0.1 \mathrm{~g} / \mathrm{L}$.

- Combination III: green filaments, assembled in a reaction cup and tetrameric red vimentin. Concentrations are $0.1 \mathrm{~g} / \mathrm{L}$ green filaments and $0.15 \mathrm{~g} / \mathrm{L}$ tetrameric red vimentin. 
For all three combinations we observe the exchange of subunits at $37^{\circ} \mathrm{C}$ at the given concentrations. For observation samples are diluted into assembly buffer by a factor of 20. The dilution slows the assembly and exchange processes in the sample and also provides a microscopy image sufficiently sparse to distinguish individual filaments. Samples are regularly taken every 60 minutes. However, for individual experiments we have also taken samples every 15 minutes. Combination III is also observed at room temperature $\left(\approx 20^{\circ} \mathrm{C}\right)$. All other parameters remain identical. Imaging parameters are described in chapter 3.5.2.

\subsection{Soft lithography}
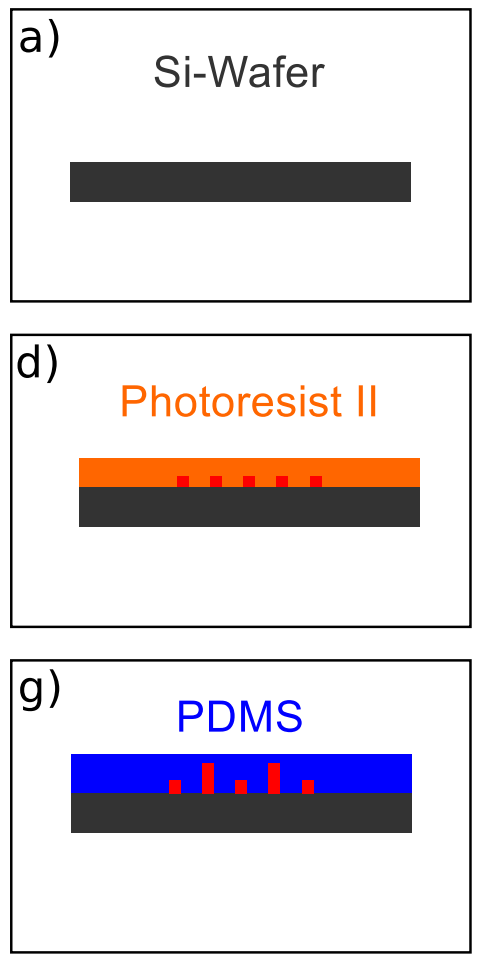
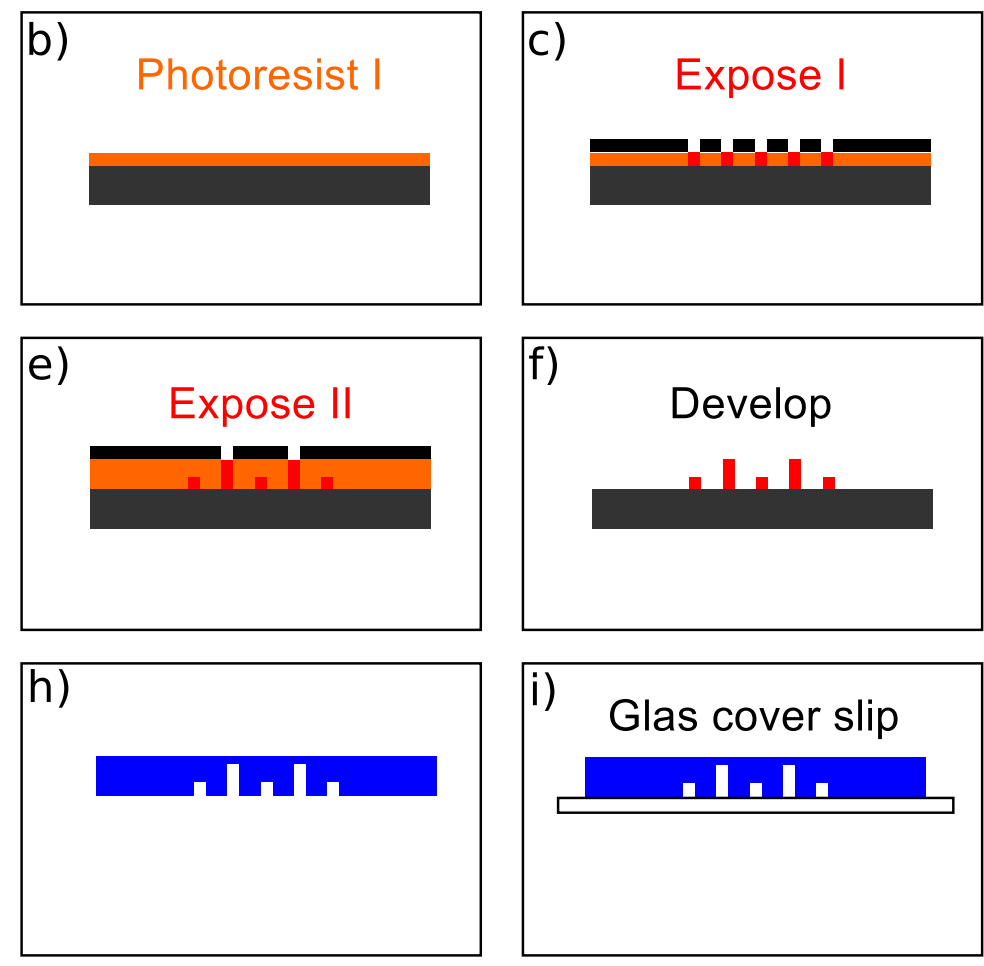
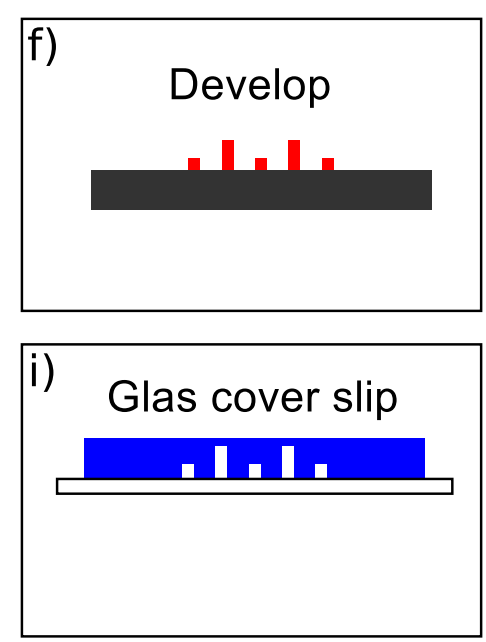

Figure 3.1: Soft lithography steps for the fabrication of a dual height microfluidic device. a) Cleaned silicon wafer. b) Wafer coated with first layer of photo resist. c) The wafer is exposed through the first photomask. d) A second layer of photo resist is applied on the first layer. e) The resist is exposed through a second photomask. f) The photoresist is developed. g) PDMS is poured on the wafer and resist structure. h) The PDMS is peeled from the wafer. i) The PDMS slap is bonded with a glass cover slip.

The microfluidic devices used for our experiments are produced using soft lithography methods 60,61]. In short, the negative of the desired channel layout is structured on a silicon wafer using SU8 photoresists. With this master stamp, 
molds are formed from PDMS (poly(dimethysiloxane), $\left.\mathrm{CH}_{3}\left(\mathrm{SiO}\left(\mathrm{CH}_{3}\right)_{2}\right)_{n} \mathrm{Si}\left(\mathrm{CH}_{3}\right)_{3}\right)$, which in turn are sealed with a glass cover slip to form the actual device.

\subsubsection{Photolithography}

For the production of microfluidic devices we used SU8 photoresist (MicroChem, Newton, MA, USA) and standard photolithography methods 62, 63 because of the resist's good tolerance of physical stress and generally good handling. As the resist height is mainly determined by the percentage of solids and the viscosity of the resist, we use different resist formulations for our purposes. Layer thicknesses of 1,5 and $25 \mu \mathrm{m}$ can be produced with commercially available formulations. A layer thickness of approximately $0.5 \mu \mathrm{m}$ is obtained by mixing SU8 2001 resist in a one to one ratio with SU8 2000 thinner (cyclopentanone) and thus reducing the amount of solids in the resist by a factor of 2 . We find the actual layer height to be $0.45 \mu \mathrm{m}$. This deviation from our estimate can be attributed to the change in viscosity of the resist mixture. In the following we will refer to this mixture of thinner and resist as SU8 "2000.5". Parameters for the production of different resist

\begin{tabular}{l||llll} 
Resist & $\begin{array}{l}\text { Height } \\
{[\mu \mathrm{m}]}\end{array}$ & $\begin{array}{l}\text { Softbake } \\
{[\mathrm{min}]}\end{array}$ & $\begin{array}{l}\text { Exposure } \\
{\left[\mathrm{mW} / \mathrm{cm}^{2}\right]}\end{array}$ & $\begin{array}{l}\text { Development } / \\
\text { Postbake }[\mathrm{min}]\end{array}$ \\
\hline \hline SU8 “2000.5" & 0.45 & 1 & 45 & 2 \\
SU8 2001 & 1 & 1 & 45 & 2 \\
SU8 3005 & 5 & 3 & 100 & 3 \\
SU8 3025 & 25 & 15 & 200 & 15
\end{tabular}

Table 3.2: Parameters for the production of SU8 photoresist layers of different heights. Spincoater as well as hard-bake parameters are identical for all resists.

layer thicknesses can be found in table 3.2. The values we used are based on the manufactures protocols found in reference [64 and adapted to ideally comply with our equipment and experimental requirements. Here, we give the protocol for the fabrication of a dual height PDMS device. The protocol can easily be adapted for the fabrication of a single height device. First, a wafer is cleaned with isopropanol and kept at $200^{\circ} \mathrm{C}$ for 10 minutes to evaporate water residues (figure 3.1 a)). Then $1 \mathrm{~mL}$ of resist is deposited in the center of the wafer. The resist is distributed at a spin speed of $500 \mathrm{rpm}$ for $10 \mathrm{~s}$ with an acceleration rate of $100 \mathrm{rpm} / \mathrm{s}$. The resist thickness is determined by the final spin speed, which for our purposes is always set 
to $3000 \mathrm{rpm}$ for $30 \mathrm{~s}$ with an acceleration of $300 \mathrm{rpm} / \mathrm{s}$ (figure 3.1 b)). Now, the softbake at a temperature of $95^{\circ} \mathrm{C}$ begins. After the softbake, the wafer is placed on the mask aligner (MJB4, Suess MicroTec, Garching, Germany) and exposed at a wavelength of $365 \mathrm{~nm}$ through either a glass-chrome photomask (ML\&C, Jena, Germany) or photoemulsion-on-film mask (Selba S.A., Versoix, Switzerland). The process is illustrated in figure 3.1 c)). Depending on the desired feature sizes, different contact modes can be used. For feature sizes of about $1 \mu \mathrm{m}$, vacuum contact mode ensures best results. For larger feature sizes of about $5 \mu \mathrm{m}$ hard contact mode can be used. After the first exposure a second layer of resist is deposited on the first (figure $3.1 \mathrm{~d}$ )). The softbake process is repeated with bake times adjusted to the second resist layer height. After the softbake the resist is exposed again (figure 3.1 e)). Here, it is important to align the already exposed structures with the second photomask. Otherwise the structures of the first and second exposure will not overlap perfectly. After the second exposure the postbake step takes place at $95^{\circ} \mathrm{C}$. Postbake times are adjusted for the final resist thickness on the basis of [64]. After the postbake step the wafer is placed in development solution (MR-Dev-600, Micro Resist Technologies GmbH, Berlin, Germany) (figure 3.1 f)). To improve the binding between wafer and developed resist, the wafer is placed on a heating plate at $150^{\circ} \mathrm{C}$ for 10 minutes.

To produce microfluidic channels with only one channel height, we simply skip the application of the second resist layer and the respective softbake and exposure steps.

\subsubsection{PDMS device}

The wafer with the negative of the channel structure is coated with (heptafluoroprobyl)-trimethylsilane (Aldrich, Steinheim, Germany) to facilitate easier removal of cured PDMS. A mixture of PDMS and cross-linker (Sylgard 184, DowCorning, Midland, MI, USA) is prepared in a 8:1 ratio and poured onto the master wafer (figure $3.1 \mathrm{~g})$ ). The wafer is then placed in a desiccator to remove air bubbles. The air bubble free PDMS is then cured at $65^{\circ} \mathrm{C}$ for at least 2 hours. Then the cured PDMS is carefully removed from the wafer (figure 3.1 h). When handled with care, the wafer can be used numerous times to fabricate new PDMS molds.

The PDMS mold is treated according to the requirements of the further experiments. Holes for tubing are punched for diffusion chamber devices. Finally, the PDMS is bonded to a glass cover slip (figure 3.1 i)). For this purpose, both the 
PDMS slab and the cover slip are placed in a plasma cleaner (PDC-32G, Harrick Plasma, Ithaca, New York, USA) for 12 seconds. Afterwards they are firmly joined together. The thus formed bond does not break under normal circumstances and can withstand static pressures of up to 2 bar. This makes it possible to use the microfluidic device with syringe pumps and flow speeds of about $1 \mathrm{~mm} / \mathrm{s}$.

\subsection{Microfluidic devices}
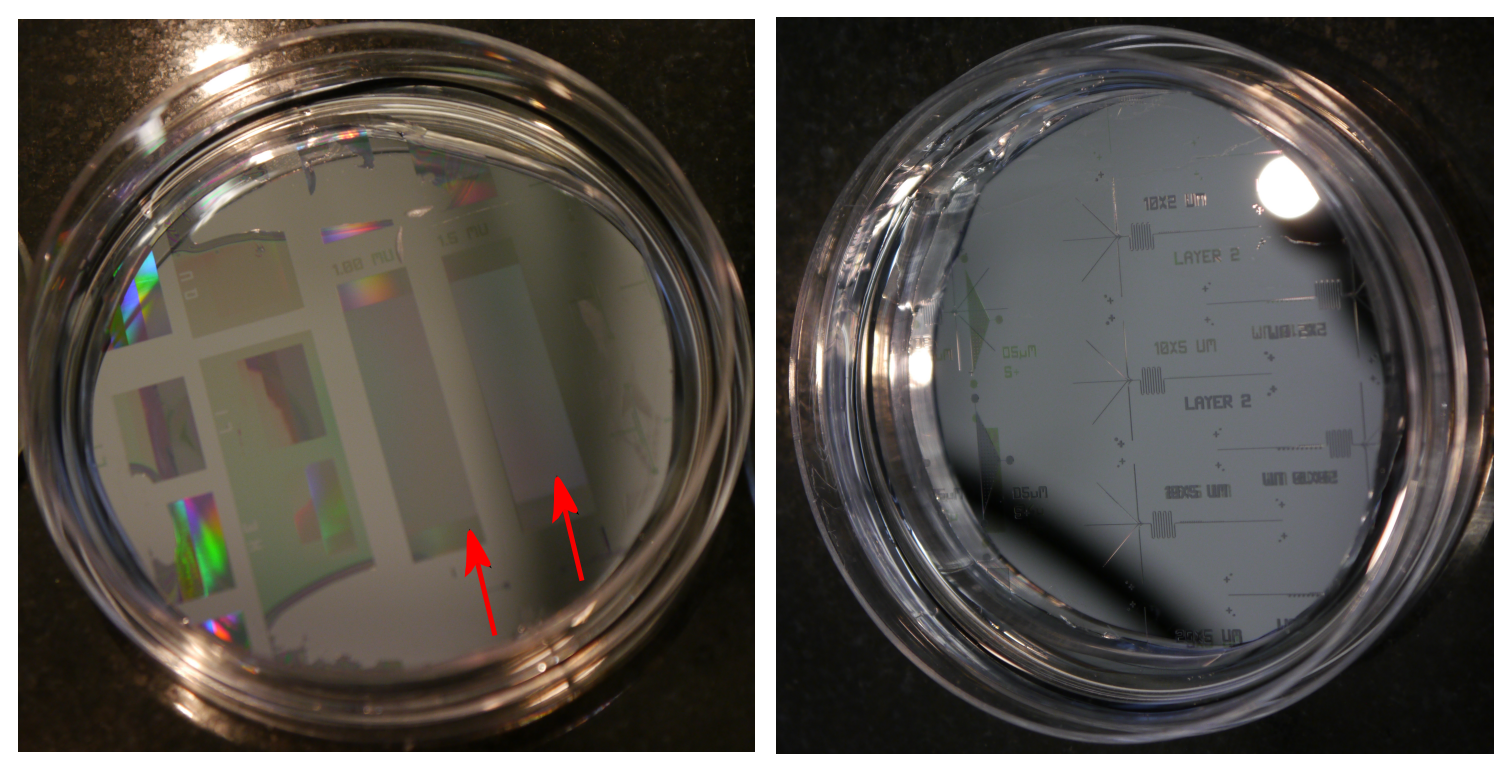

Figure 3.2: Wafers for the production of PDMS devices: (left) Parallel channels for observation of vimentin filaments in confinement (red arrows). (right) Diffusion chamber layouts with different dimensions of connecting channels. Diameter of wafers $5 \mathrm{~cm}$.

The main advantage of microfluidic devices constructed from PDMS is the great flexibility associated with their straightforward production process. This entails an inert surface chemistry, easy reproducibility and a low costs. Different layouts can be tested and refined rather time efficiently. For our purposes, we employ two distinct classes of microfluidic devices. The first class consists of many parallel channels with a rectangular cross-section and is used for the observation of fluctuating vimentin filaments in confinement. A silicon wafer with SU8 photoresist structure is shown in figure 3.2 (left). The actual channels as observed through the microscope are shown in figure 2.5. The second class of devices are named diffusion chambers. They consist of several dead-end-chambers attached to a main channel. 
An image of a wafer with different diffusion chamber layouts is shown in figure 3.2 (right). A sketch of the device can be found in figure 3.4 .

\subsubsection{Microchannels}
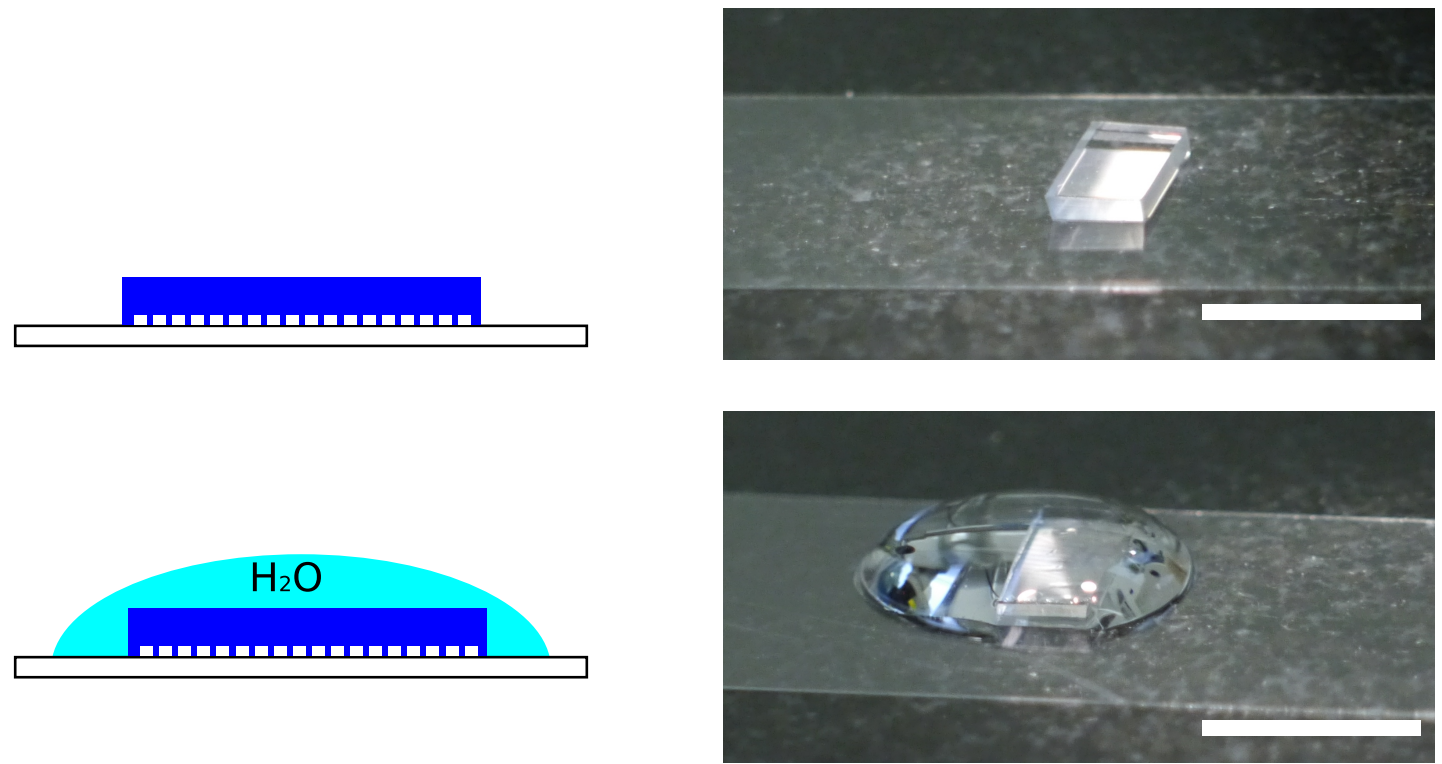

Figure 3.3: Sketch and photography of a microchannel device (top) PDMS microchannel device with glass cover slip. The small size of the device enables easy filling of the channels via capillary effect. (bottom) Microchannel device covered in buffer to prevent induced flow in the microchannels. Scale bar shows $10 \mathrm{~mm}$

For the analysis of fluctuating vimentin filaments we produce narrow channels with a rectangular cross-section. The channels have a height of either $0.45 \mu \mathrm{m}$ or $1 \mu \mathrm{m}$, obtained by using SU8 "2000.5" or SU8 2001 photoresist, respectively. Channels of both heights are produced with three widths each: $1.2 \mu \mathrm{m}, 1.6 \mu \mathrm{m}$ and $2.7 \mu \mathrm{m}$. To increase the chance to observe an isolated filament in a single frame, many parallel channels are placed next to each other. Through careful optimization we have found the lithography protocols detailed in chapter 3.3.2, which ensure a rectangular shape of the channel cross-section.

The actual experiment to observe individual vimentin filaments in microchannels is started with a freshly assembled microfluidic device. Capillary forces are used to flush in a solution of $50 \mathrm{mg} / \mathrm{mL}$ BSA. This prevents the adsorption of vimentin filaments to the channel walls. Further details on the surface treatment techniques we used can be found in chapter 3.4. After flushing with BSA solution, left over solution is removed from the outside of the device with a tissue. Then a drop of 
vimentin filament solution is placed in front of the channels and enters them through capillary forces. The vimentin concentration in solution is $1 \mathrm{mg} / \mathrm{L}-5 \mathrm{mg} / \mathrm{L}$.

A characteristic property of PDMS is the permeability to both water and air [65]. While the permeability to air is mostly to our advantage, for instance when a dead end chamber needs to be filled with solution, the permeability to water is typically not desired. If a channel is filled with aqueous solution, some solution is "lost" into the PDMS through diffusion. This fluid "loss" induces a flow in the microchannels [66]. Especially in the context of the fluctuation analysis we perform on vimentin filaments in these channels, flow would disturb our measurements. The most practical solution to prevent flow in the device is to soak the PDMS in water prior and during use. The PDMS is then unable to absorb more water and flow is absent after an equilibration phase. In the case of the microchannel device we simply cover the whole PDMS device with a drop of phosphate buffer to stop induced flow. An image of an immersed device is shown in figure 3.3 .

After the equilibration phase, vimentin filaments are fluctuating in the microchannels without significant movement along the channels. At this point we begin to image the filaments. Imaging parameters are listed in chapter 3.5.1.

\subsubsection{Diffusion chambers}

Diffusion chamber devices are also constructed from PDMS in a very similar manner to the microchannels described above. A sketch of the device layout is shown in figure 3.4. The chambers with diameters from 10 to $200 \mu \mathrm{m}$ are connected to a main channel with a width of $50 \mu \mathrm{m}$ via small channels. The channel and chamber height is $25 \mu \mathrm{m}$. The dimensions of the connecting channels determine the typical diffusion timescales. And we can thus regulate the times the reactants in the main channel and the chambers need to equilibrate. Typically, we employ connecting channels with a size of $10 \mu \mathrm{m}$ by $10 \mu \mathrm{m}$. We use finite element simulations to determine the expected equilibration times, as described in chapter 5.1 .

The microfluidic device is connected to a syringe actuated by neMESYS high precision syringe pumps (Cetoni GmbH, Korbussen, Germany) with polyethylene tubing. These syringe pumps allow us to apply precise flow rates of up to five different solutions. The experiments with diffusion chambers can be divided into different phases, in which different solutions are flushed through the device. The individual duration of each phase has been either determined by evaluating diffusion times with finite element simulations or the observation of an actual experiment. The 


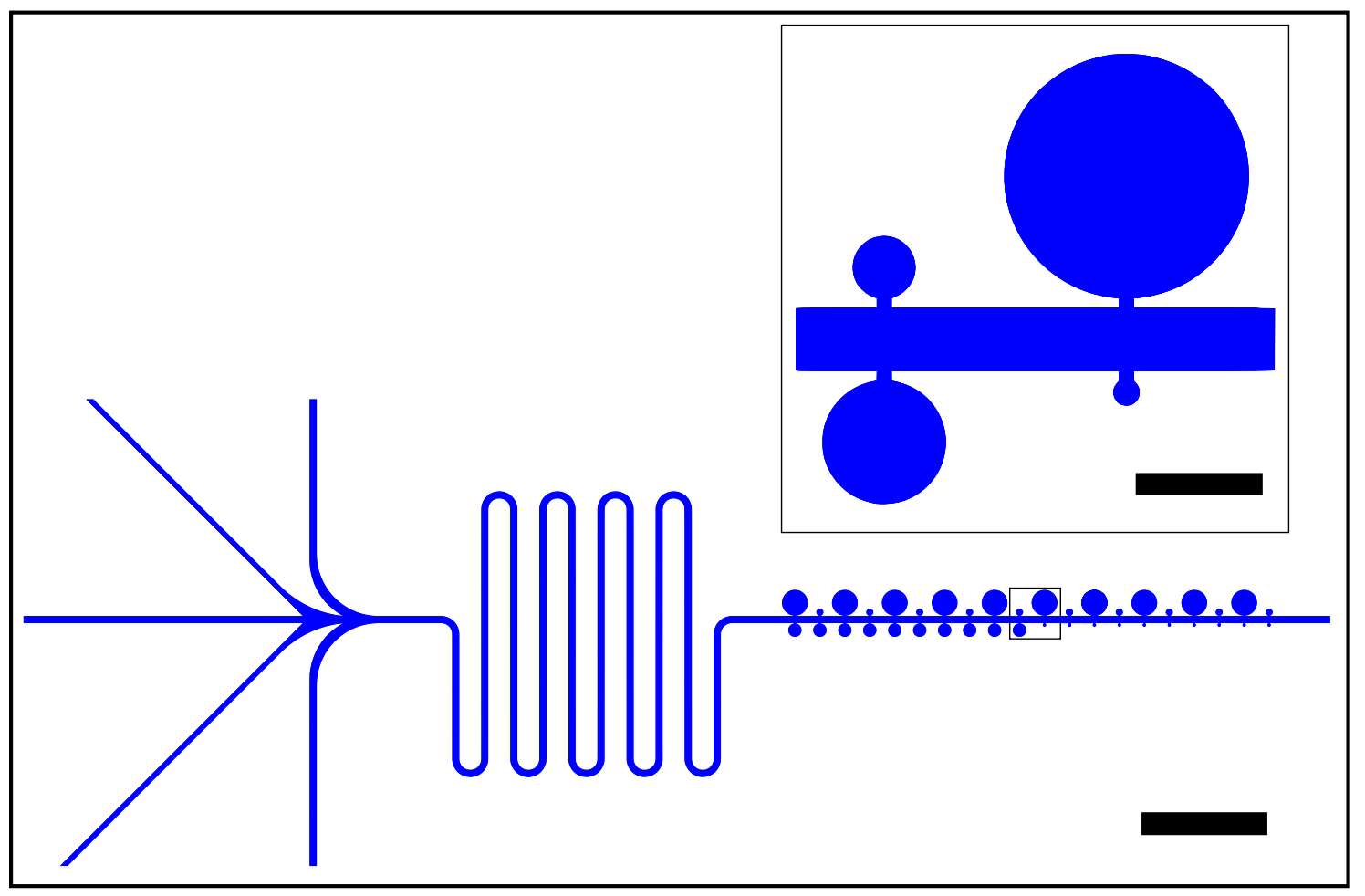

Figure 3.4: Sketch of a diffusion chamber device. From left to right we see the five inlets, serpentines for diffusive mixing of buffers, then the actual chambers and a single outlet. As shown in the inset, the chambers are connected to the main channel with $10 \mu \mathrm{m}$ by $10 \mu \mathrm{m}$ connecting channels. Scale bars show $100 \mu \mathrm{m}$ (inset) respectively $1000 \mu \mathrm{m}$.

phases are the following:

1. Coating of the device to passivate the surfaces with regards to vimentin. Different methods for surface passivation are listed in chapter 3.4 . For the most effective coating, we flush with small unilaminar lipid vesicles at $50 \mu \mathrm{L} / \mathrm{h}$. A lipid bilayer is thus established on the device surface. As shown in figure 3.5 the formation of a closed lipid bilayer is observed after 30 minutes.

2. The device is flushed with salt free buffer ( $2 \mathrm{mM}$ phoshphate buffer $\mathrm{pH} 7.5)$ for 30 minutes at $50 \mu \mathrm{L} / \mathrm{h}$ to remove residual lipid vesicles from the main channel and the chambers.

3. Tetrameric vimentin $(0.4 \mathrm{~g} / \mathrm{L}$, AlexaFluor 488) is flushed in through the main channel at $50 \mu \mathrm{L} / \mathrm{h}$. After 60 minutes concentrations in the main channel and largest chambers $(200 \mu \mathrm{m})$ have equilibrated.

4. The channel is flushed with $1 \mu \mathrm{L}$ salt free buffer to remove the tetrameric vimentin and prevent the formation of vimentin aggregates in the channel. 
5. Assembly is initiated by flushing with $100 \mathrm{mM} \mathrm{KCl}$ buffer at $50 \mu \mathrm{L} / \mathrm{h}$. Assembly times can be varied. However, we typically let the assembly process continue for 180 minutes.

6. We flush the channel with $1 \mu \mathrm{L}$ salt free buffer at $50 \mu \mathrm{L} / \mathrm{h}$ to remove the $100 \mathrm{mM} \mathrm{KCl}$ buffer. This prevents clogging of the main channel by vimentin assembly in the next step.

7. Tetrameric vimentin $(0.4 \mathrm{~g} / \mathrm{L}$, ATTO $647 \mathrm{~N})$ is flushed through the main channel at $50 \mu \mathrm{L} / \mathrm{h}$ for 30 minutes. The protein can thus enter the chambers by diffusion.

8. We flush with $1 \mu \mathrm{L}$ salt free buffer to prevent assembly of vimentin in the main channel.

9. The assembly of both vimentin species is resumed/started by flushing with $100 \mathrm{mM} \mathrm{KCl}$ buffer at $50 \mu \mathrm{L} / \mathrm{h}$. Typically the assembly reaction is observed for 120 minutes, but this observation time can be rather freely changed.

10. Lastly, we can flush with salt free buffer at $50 \mu \mathrm{L} / \mathrm{h}$ which should start a disassembly reaction in vimentin filaments.

If we only want to observe vimentin labeled with one fluorescent dye, we omit steps 6 to 9. Also, when for one reason or another only epi-fluorescence microscopy imaging is desired, it is possible to reduce the overall height of the chambers to $5 \mu \mathrm{m}$ and at the same time retain the height of $25 \mu \mathrm{m}$ for the main channel. By reducing the chamber height, fluorescent background is strongly reduced. At the same time, the device remains easy to handle because of the normal main channel height. To obtain these two height devices, the wafer production method has to be adapted as detailed in chapter 3.2.1. The steps following the actual wafer production procedure remain unchanged.

\subsection{Surface treatment of microfluidic devices}

In general, proteins stick to glass and other surfaces when no countermeasures are taken 67, 68. Vimentin is no exception to this. Therefore, the surface treatment of the microfluidic device is a central part of each microfluidic experiment. We employ several different methods to prevent sticking of vimentin filaments to the surfaces 
of the microfluidic devices. The different surface treatments tested and used during this thesis are listed and described below. An overview of common methods to prevent protein adsorption in microfluidic devices can be found in references [44] and 45$]$.

\subsubsection{Bovine Serum Albumin}

Bovine Serum Albumin (BSA) (CarlRoth, Karlsruhe, Germany) is an easily available protein extracted from cow blood. To passivate surfaces in regards to vimentin filaments, a solution of $50 \mathrm{mg} / \mathrm{mL}$ BSA in $2 \mathrm{mM}$ phosphate buffer at $\mathrm{pH} 7.5$ is flushed through a freshly sealed microfluidic device and incubated for 5 to 15 minutes. During this process BSA adsorbs to the surface and thus forms a protective structure. Direct interaction of vimentin with sticky PDMS or glass surfaces is prevented or at least reduced. Typically this treatment prevents vimentin filaments from adhering to surfaces for about 15 minutes.

A slightly different application of this method is the direct introduction of BSA into a solution already containing vimentin filaments. This approach has the advantage that no separate anti-stick treatment of the microfluidic devices is necessary. However, in this case vimentin filaments and BSA compete for binding places on any available surfaces. Consequently, the direct mixing of filaments and BSA is less effective than a treatment with only BSA and consecutive introduction of filament solution.

The main advantages of this method are easy availability and application as well as good anti-stick properties in the short term. However, long term use is not possible in most cases. This makes the surface treatment with BSA an ideal candidate for our experiments on individual vimentin filaments in microchannels shown in chapter 6. Here, we require a surface coating for a duration of 15 minutes, which is provided by a treatment with BSA.

\subsubsection{PLL-g-PEG}

PLL-g-PEG consists of a poly-lysine backbone on which polyethylene glycol chains are grafted. We use PLL(20)-g[3.5]-PEG(2) (SuSoS, Dübendorf, Switzerland) 46, 69. This compound consists of PLL chains with a molecular weight of $20 \mathrm{kDa} .3$ to 4 PEG chains with a molecular weight of $2 \mathrm{kDa}$ each are then grafted onto the PLL chain. When the PLL-g-PEG solution comes into contact with a freshly plasma 
cleaned surface like glass or PDMS, the PLL backbone binds to this surface and the PEG chains form a tight brush-like surface. These brush-like PEG chains offer little opportunity for proteins to attach to surfaces.

A solution of $1 \mathrm{mg} / \mathrm{mL}$ PLL-g-PEG in $2 \mathrm{mM}$ phosphate buffer, $\mathrm{pH} 7.5$ is stored at $-20^{\circ} \mathrm{C}$ ready for use. This solution is flushed through a freshly plasma cleaned and sealed microfluidic device for 15 minutes. The coating with PLL-gPEG should yield a very uniform and well defined surface as well as good anti-stick properties for several hours. However, the application of a PLL-g-PEG coating to diffusion chambers as shown in chapter 3.3 .2 has proven unsuitable for the assembly of vimentin filaments. Thus the treatment with PLL-g-PEG is not the ideal surface treatment for our experimental systems.

\subsubsection{Sigmacote}

Sigmacote (Sigmar Aldrich, Munich, Germany) is a commercially available surface treatment product which in the combination with glass cover slips gives very good anti-stick properties concerning vimentin filaments. However, the application of sigmacote in PDMS devices resulted in clogged channels and diffusion chambers as well as changes in the appearance of the PDMS near the channels. Therefore, despite very promising prospects, we do not use sigmacote as a surface treatment in our experiments.

\subsubsection{Lipid bilayer}

A lipid Bilayer which covers the PDMS and glass surfaces in the device can be obtained by flushing a lipid vesicle solution through the device [70,71]. The preparation of the lipid vesicle solution is detailed below. In the case of diffusion chambers with a diameter of $200 \mu \mathrm{m}$ it takes 30 minutes to form a closed lipid bilayer, as seen in figure 3.5. To supply new vesicles, the flow in the microfluidic device remains constant over the whole time. The limiting factor for the spreading time of the lipid bilayer in this case is the transport of lipids from the main channel into the chamber. We use lipids which are labeled with a fluorescent dye to visualize the lipid bilayer. Therefore, we can conveniently verify the quality of the chamber surface treatment.

The application of a lipid bilayer has the advantage of very good anti-stick properties as well as excellent long time stability. This allows us to perform experiments with a duration of more than 24 hours without loosing anti-stick properties. 

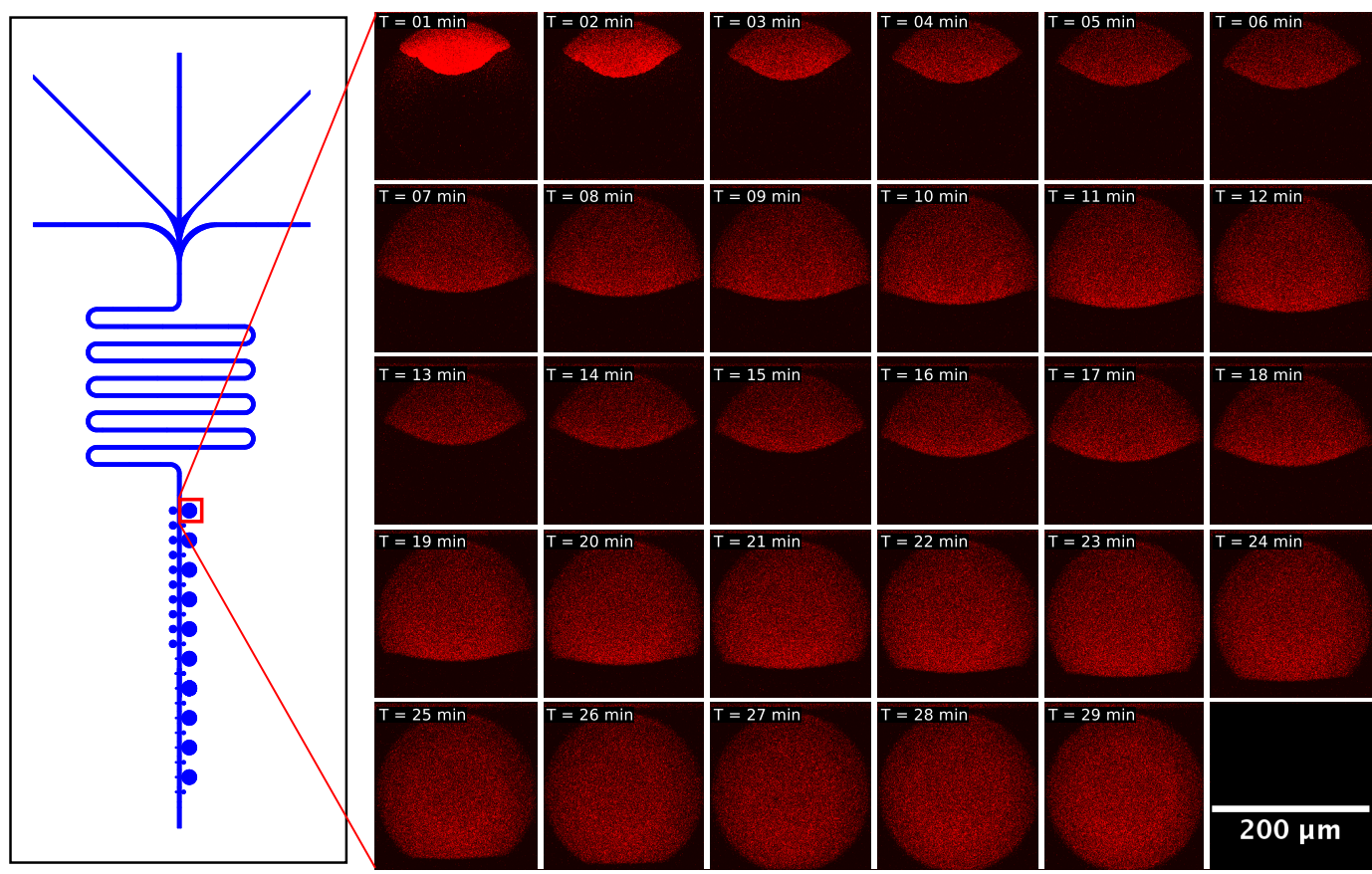

Figure 3.5: Spreading of a lipid bilayer in a diffusion chamber. Lipids labeled with TexasRed are flushed through the main channel of the diffusion chamber device and slowly enter into the chamber and spread onto the surfaces. For a chamber with a diameter of $200 \mu \mathrm{m}$ the process is completed within 30 minutes. The location of the chamber in the microfluidic device is indicated in the sketch on the left.

Apart from the anti-stick properties of this coating, the lipid bilayer closely resembles the cellular membrane. This is an additional benefit for experiments, which in the end emulate cellular processes. The only downside of this method is the comparatively complex preparation. This also entails that we need to take extra care to prevent air bubble formation in the microchannels during experiments. This is necessary because the lipid bilayer is not resistant to even brief exposure to air and consecutive loss of anti-stick properties.

\subsubsection{Lipid vesicle preparation}

Small unilaminar vesicles (SUVs) are produced from 98\% 1,2-dioleoyl-sn-glycero3-phosphocholine (DOPC) (Avanti) and 2\% 1,2-diheptanoyl-sn-glycero-3-phosphocholine (DHPC) (Invitrogen) labeled with TexasRed dye. Stock solutions of $10 \mathrm{mM}$ DOPC in $\mathrm{CHCl}_{3}$ and $1 \mathrm{mM} \mathrm{DHPC}$ in $\mathrm{CHCl}_{3}$ are combined such that a total of $25 \mathrm{mg}$ of lipids are contained in solution. For the given ratio of DOPC to DHPC this results in $25 \mu \mathrm{L} \mathrm{DOPC}$ and $9 \mu \mathrm{L}$ of labeled DHPC. This solution is deposited in a small glass vial. The sides of the vial are rinsed with $\mathrm{CHCl}_{3}$. The $\mathrm{CHCl}_{3}$ is 
evaporated under a weak stream of nitrogen for about 30 minutes. After that the drying process is completed under vacuum at $40^{\circ} \mathrm{C}$. If other lipids are used, the temperature has to be adjusted to be above the phase transition temperature of the lipids. After this step, dried lipid layers are deposited at the bottom of the glass vial. These dried lipid layers can be stored for up to 6 months at $4^{\circ} \mathrm{C}$ under a nitrogen atmosphere.

To actually obtain vesicles, the dried lipids are dissolved in $2 \mathrm{mM}$ phosphate buffer pH 7.5. Typically the buffer volume is chosen so that the lipid concentration in solution is $100 \mathrm{mg} / \mathrm{ml}$. The dissolving process of the lipid layers can be accelerated by alternately heating the solution to $50^{\circ} \mathrm{C}$ and vortexing it. After the lipids are completely dissolved, two basic methods are available to produce SUVs. First, by repeatedly squeezing the lipid solution through a membrane with pore sizes of about $50 \mu \mathrm{m}$, vesicles of diameters as small as the pore sizes are extruded. A second method can be used to obtain vesicles with diameters in the range of $5 \mu \mathrm{m}$ to $10 \mu \mathrm{m}$. Here, the lipid solution is placed in a probe sonicator (Sonopuls UW 2070, Bandelin, Berlin, Germany) for 20 minutes at $50 \%$ intensity and a $10 \%$ duty cycle. To prevent excessive temperature increase in the sample, the reaction tube is placed in a water bath during the sonication process. As a last step, titanium particles originating from the probe sonicator are removed by centrifuging the vesicle solution at $12100 \mathrm{~g}$ for 30 minutes and discarding the sediment. The vesicle solution obtained in this manner can be stored for approximately 1 week at $4^{\circ} \mathrm{C}$ prior to use. After this time the vesicles fuse into larger vesicles, which can in turn be "refreshed" by once again probe-sonicating the sample.

We use probe sonicated SUVs for our experiments. The thus produced smaller vesicles tend to spread faster onto surfaces because of increased surface tension for smaller diameters. Also, the amount of vesicle solution needed for our purposes is very small and the extrusion of lipid vesicles through a membrane produces a comparatively large dead volume.

\subsection{Microscopy}

Individual vimentin filaments with diameters of about $10 \mathrm{~nm}$ are imaged with different fluorescence microscopy methods described in detail below. Naturally, samples must be fluorescently labeled when they are observed with fluorescent microscopy. The labeling process for vimentin can be found in chapter 3.1.3. In the following 


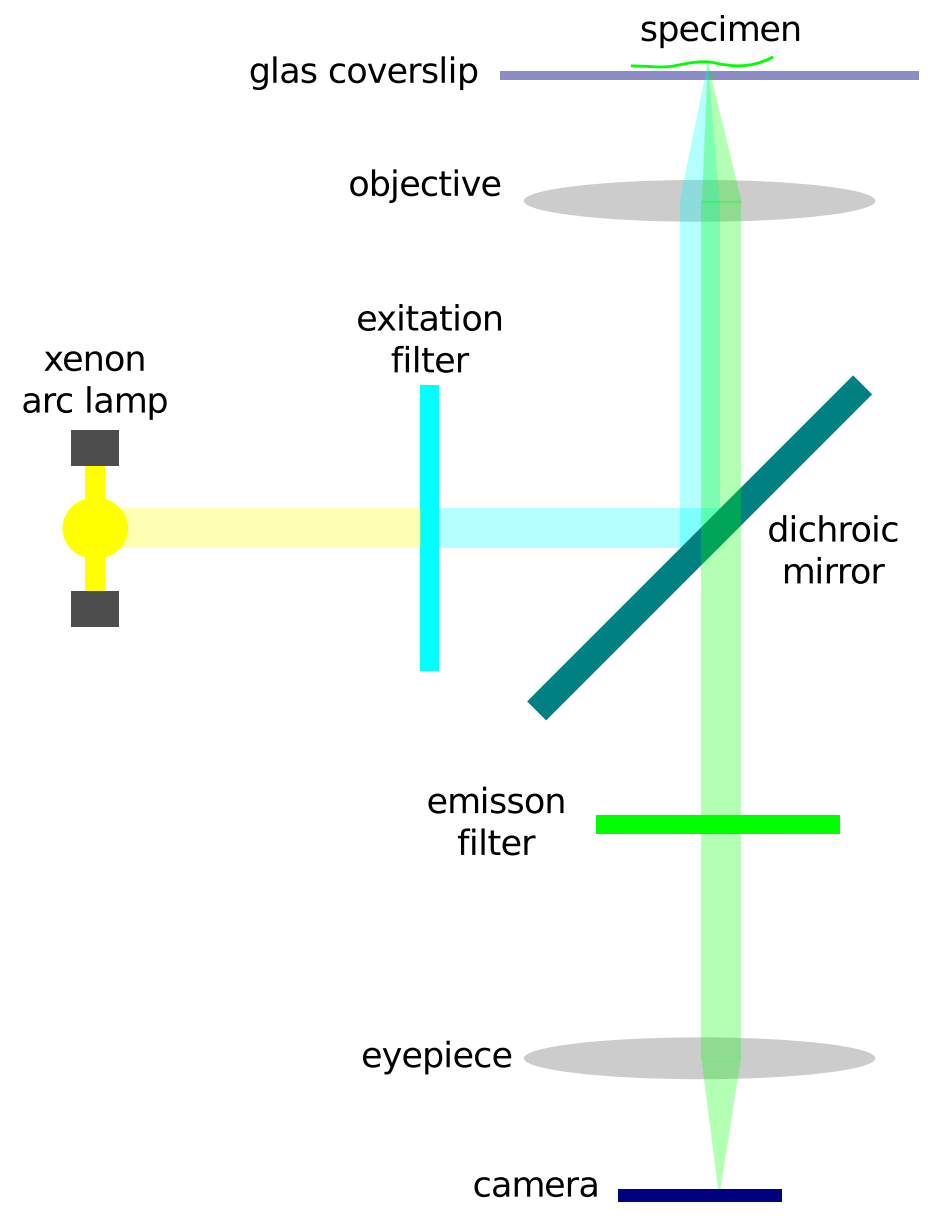

Figure 3.6: Sketch of the beam-path of an epi-fluorescence microscope. The specimen, which is labeled with a fluorescent dye, is illuminated at a specific excitation wavelength through the microscope objective. In turn fluorescent light is emitted and collected in the objective. The collected light passes through filters to remove reflected excitation and other background light of different wavelengths. Finally the image of the specimen is projected on a CCD or CMOS camera.

we will limit the description to the fluorescence techniques used by us on an IX81 inverted microscope (Olympus, Hamburg, Germany).

Microfluidic structures can be imaged with standard brightfield microscopy. The brightfield microscope setup is based on an IX 81 inverted microscope.

\subsubsection{Epi-fluorescence microscopy}

In epi-fluorescence systems, as illustrated in figure 3.6, a certain area of the specimen is illuminated with light of suitable wavelength(s). For this purposes we use a $150 \mathrm{~W}$ xenon arc lamp as illumination. The light is directed onto a so-called filter cube (U-MNIBA3, Olympus, Hamburg, Germany), which combines filters to 




Figure 3.7: Spectral properties of the U-MNIBA3 filter cube. The graph shows the transmission values for the excitation (blue) and emission (red) filters as well as the values for the dichroic mirror (green). Image from 72

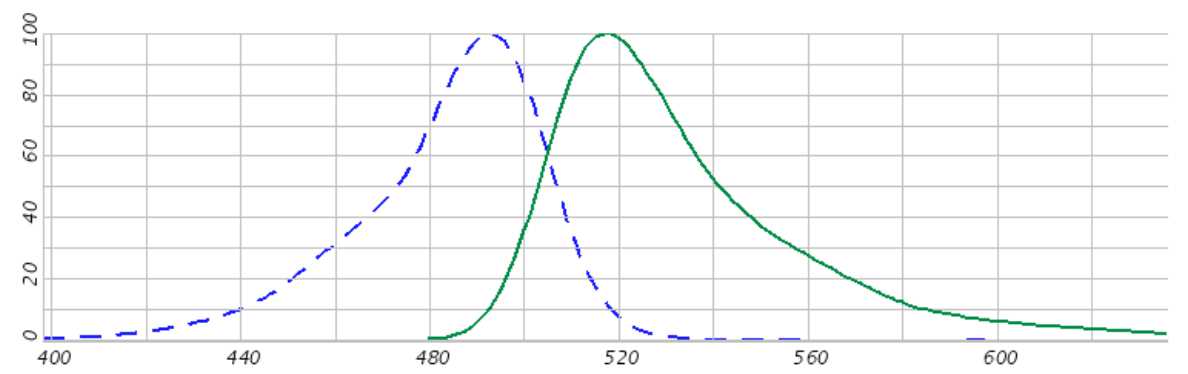

Figure 3.8: Absorption and emission of AlexaFluor 488 fluorescent dye. The excitation spectrum is shown in blue, the emission spectrum in green. The difference in wavelengths between the maximum of both curves is called stokes shift. Image from [55]

select the excitation and emission wavelengths as well as a dichroic mirror with a corresponding cut off wavelength. Spectra for the filtercube can be found in figure 3.7. In the filter cube wavelengths for fluorescence excitation are selected and transmitted on the sample through the objective. We use a $60 \mathrm{x}$ oil immersion objective (UPLSAPO 60XO, Olympus, Hamburg, Germany) with a N.A. of 1.35. The sample in turn emits light with a longer wavelength, which is collected in the objective. The spectrum of the dye AlexaFluor 488 is shown in figure 3.8 as a general example, the situation for different dyes is comparable and therefore not explicitly shown. The fluorescence light then passes through the dichroic mirror and the emission filter, which remove undesired wavelengths such as the excitation wavelength. Finally, the specimen is imaged using a CCD-camera (ORCA-R2, Hamamatsu Photonics Deutschland GmbH, Herrsching am Ammersee, Germany). For this thesis, we use the epi-fluorescence system only to image vimentin labeled with AlexaFluor 488. 
Imaging parameters vary with the specimen and the desired timescales like total observation time and frame rate. Typically, for individual filaments confined in microchannels, we use an acquisition time of $50 \mathrm{~ms}$ and a frame rate of $10 \mathrm{~Hz}$ and a total number of frames from 300 to 600 .

\subsubsection{Confocal microscopy}

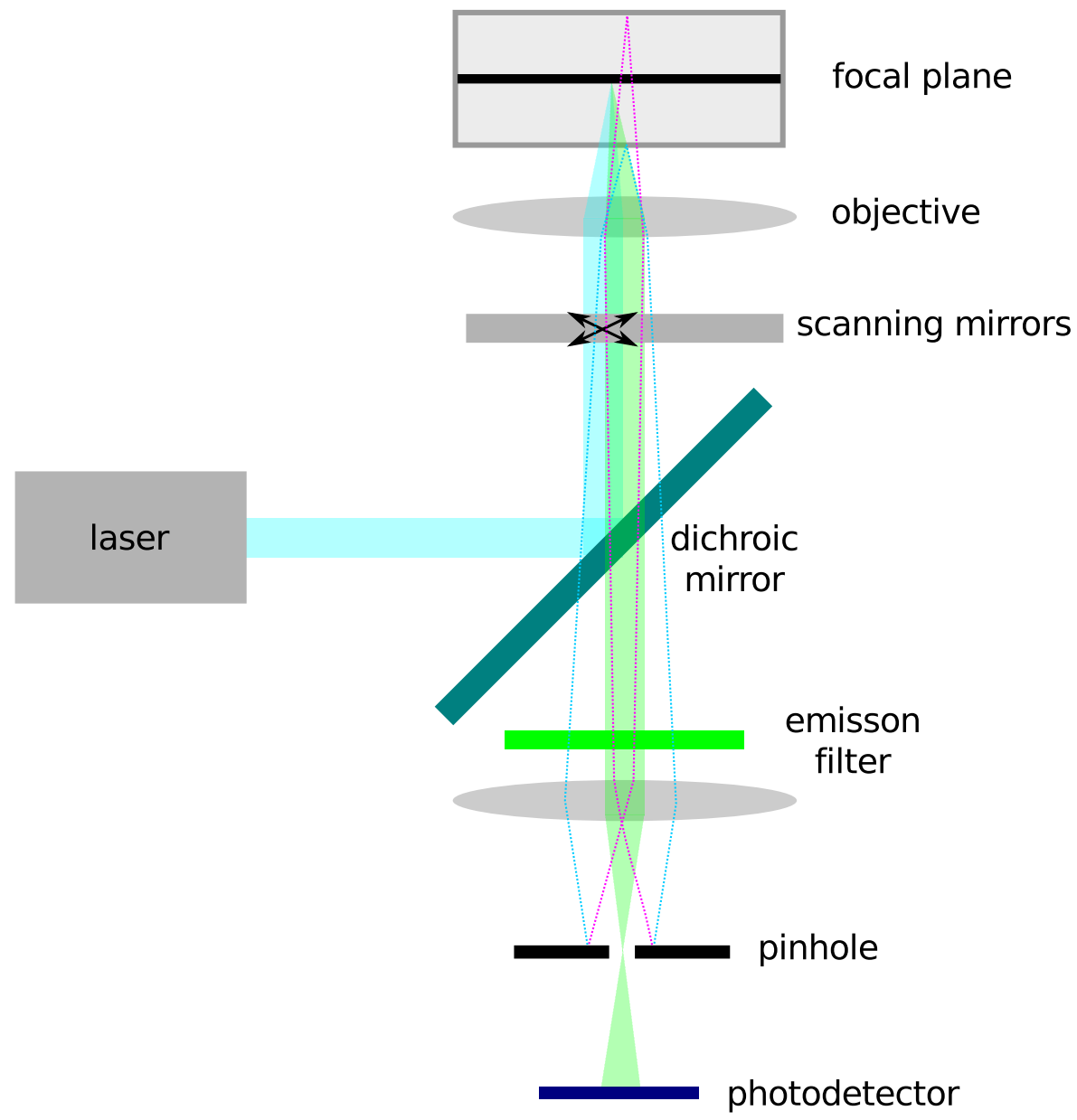

Figure 3.9: Sketch of the beampath in a confocal microscope The principal imaging mechanism is similar to that of the epi-fluorescence microscope shown in figure 3.6. However, the sample is illuminated only pointwise in a scanning pattern. Also the emitted light is filtered with a pinhole. This filtering process reduces background signal from outside the focal plane.

Confocal microscopy relies on the same basic principles as epi-fluorescence microscopy. The major change is that not a single, large area of the specimen is illuminated at once, but instead the specimen is imaged pointwise in a scanning pattern. Because only single points are imaged, it is now possible to exclude elements, 

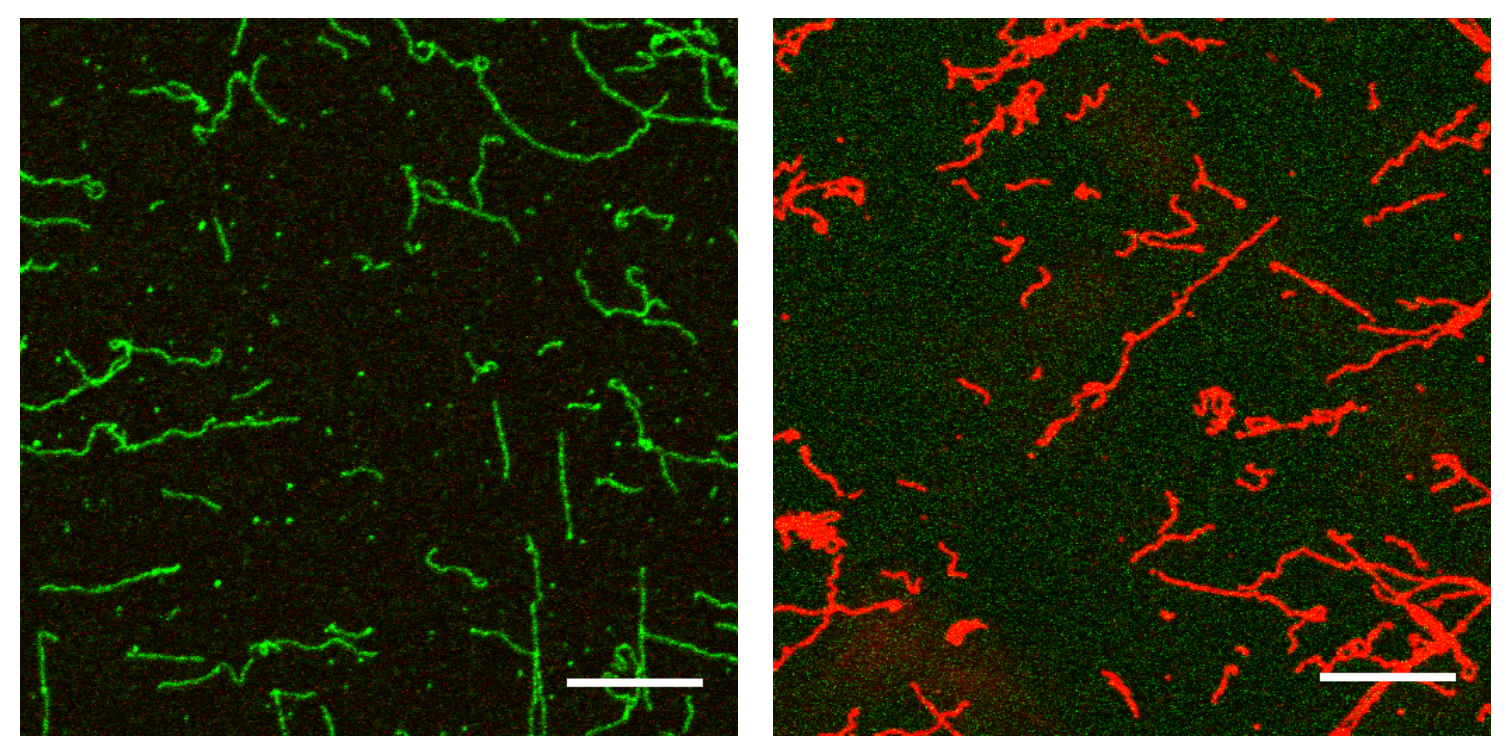

Figure 3.10: Fluorescence image of vimentin filaments labeled with Atto 647N and AlexaFluor 488. Two images are taken on the same sample region: (left) Excitation only at $488 \mathrm{~nm}$, detection in both channels. (right) Excitation only at $635 \mathrm{~nm}$, detection in both channels. Despite showing only the bottom quarter of the histogram in the red respectively green channel, no cross-talk is visible. Scale bars $10 \mu \mathrm{m}$

which are not directly in the focal plane, but still in the beampath from contributing to the image (see figure 3.9). This is made possible by inserting a pinhole in the beampath. The ability to reduce image background makes confocal microscopy techniques attractive mainly for thicker samples, where fluorescent background is often disruptive. It is important to note that in general the imaging time is much larger for confocal than for epi-fluorescence microscopy. Typically, the acquisition time is on the order of $100 \mathrm{~ms}$ for epi-fluorescence microscopy, whereas the acquisition times for focal microscopy images with a similar total pixel number are often in the range of 1 minute. Therefore, in the case of confocal microscopy we have to be careful to adjust the imaging timescales to the timescales of sample movement or change in the sample itself.

In a confocal microscopy setup, typically a laser is used as a light source. The two dyes used by us are AlexaFluor 488 and Atto 647N, which can be imaged using an argon laser with the $488 \mathrm{~nm}$ line and a diode laser with a wavelength of $635 \mathrm{~nm}$ on the IX81 FV1000 setup (Olympus, Hamburg, Germany). The objective used is a 60x silicone oil immersion objective (UPLSAPO 60XS, Olympus, Hamburg, Germany) with a N.A. of 1.3 .

A central feature of the confocal microscope is the ability to acquire each dye-channel sequentially either in each line or each frame. This measure brings 
the cross-talk between two channels to an absolute minimum. On the example of the two dyes listed above, figure 3.10 shows that there is basically no cross-talk between channels when sensible laser intensities are chosen. This feature is very important for the analysis of the subunit transfer between vimentin filaments shown in chapter 4. On the other hand, simultaneous detection in both channels can be used to observe FRET (Förster resonance energy transfer), when suitable dyes are chosen [73]. FRET describes a process where a donor fluorescent molecule in an excited state transfers energy to an acceptor fluorescent molecule in a radiation free process. The energy transfer process is heavily dependent on the distance between the two involved molecules. Typically distances between donor and acceptor do not exceed $10 \mathrm{~nm}$ [74]. 


\section{Chapter 4}

\section{Exchange of subunits}

In this chapter we present and discuss data that allows us to determine that subunit exchange between mature vimentin intermediate filaments occurs in vitro. In vitro experiments enable us to conveniently observe the inherent properties of vimentin filaments independent from cellular processes. A sketch of the proposed exchange mechanism is shown in figure 4.1. At this point it is unclear what size the exchanged subunits actually have. However, it is likely that the subunits are at least tetrameric, as no smaller aggregates occur even in $\mathrm{KCl}$ free phosphate buffer. Also, subuntis are very likely to be smaller than unit length filaments, as an "exchange" of a ULF would entail the breaking of a filament.

\subsection{Initial considerations and experimnets}

\subsubsection{Microscopy methods}

To determine the percentage of exchanged subunits we use vimentin filaments labeled with two distinct fluorescence dyes as described in chapter 3.1.5. We detect fluorophores, which are attached to subunits that are exchanged between the two different filaments. Thus we can calculate the percentage of exchanged subunits between filaments from the "exchanged" fluorescence between differently labeled filaments. Fluorescent dyes and matching microscopy excitation and emission filters are chosen so that no cross-talk between the two channels is observed. In addition to the isolated imaging of each channel, we also perform FRET measurements as described in chapter 3.5.2. Here, we only excite the dye with the lower excitation wavelength but observe emission in both channels. Preliminary experiments with filaments labeled with both AlexaFlour 488 and ATTO 647N dyes show that we do 


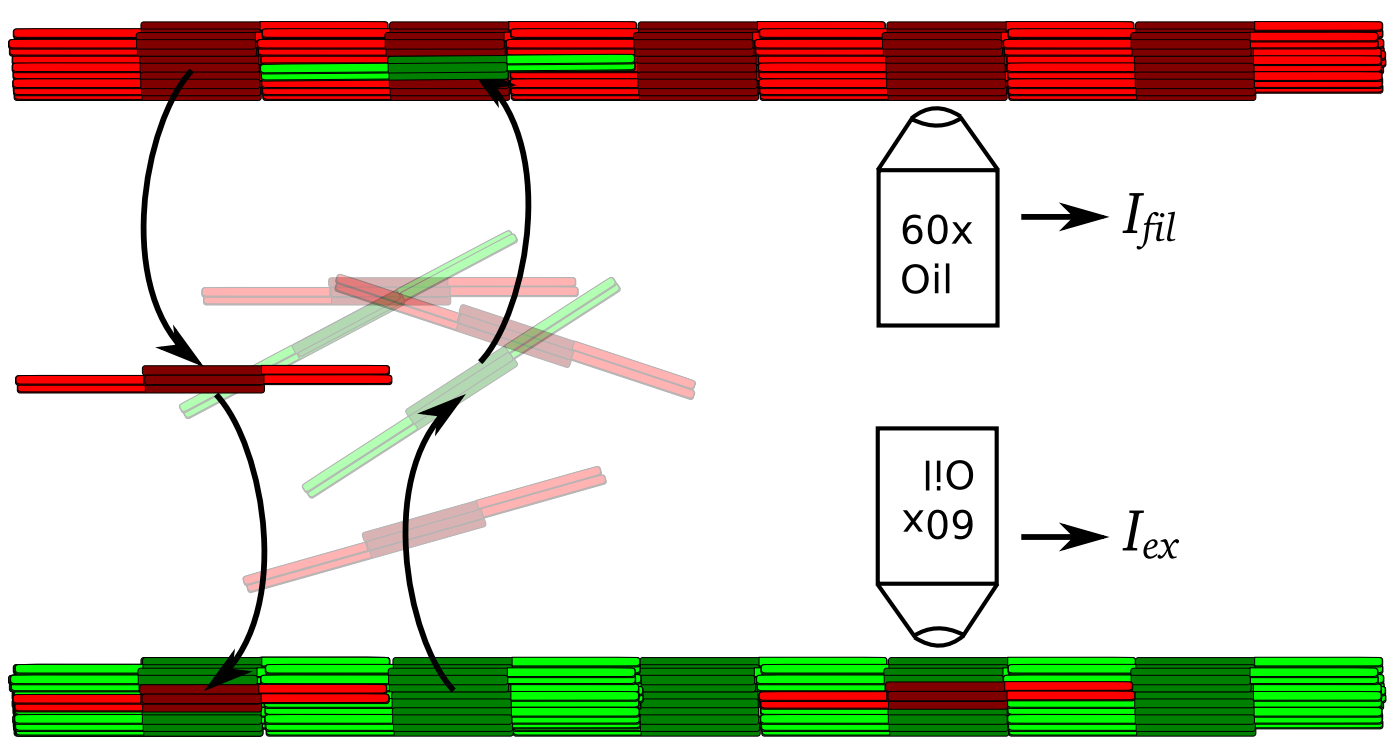

Figure 4.1: Sketch of the subunit exchange in intermediate filaments. Red subunits, here drawn as tetramers, incorporate into green filaments. This incorporation could happen in an empty "tetramer slot" or by increasing the filament cross-section locally. To calculate the percentage of exchanged filaments, we relay on the intensities of the red fluorescence at the locations of the green filaments $\left(I_{e x}\right)$ in relation to the fluorescence intensity of the red filaments $\left(I_{f i l}\right)$.

indeed detect FRET as shown in figure 4.2. However, overall image quality is superior in the case of the isolated imaging of both channels as shown in figures 3.10 and 4.3. The distinct advantage FRET measurements have over confocal microscopy measurements is the ability to determine distances between fluorophores with high precision. However, the spatial resolution of confocal microscopy images is sufficient for our purposes and we will thus restrict our measurements to this method.

As estimated in chapter 3.1 .3 the percentage of labeled tetramers is about $50 \%$. However, the exact amount of label is not directly relevant for our analysis. We only need to be certain that enough subunits carry a label to detect even a low percentage of exchanged subunits. To obtain an estimate of the number of exchanged dye molecules, we use a $1 \mu \mathrm{m}$ long filament. This filament contains approximately 200 tetrameric subunits and therefore an average of 100 dye molecules. If we want to observe a total subunit exchange of $1 \%$, a single dye molecule needs to be detected. However, filaments with a total length on the order of $100 \mu \mathrm{m}$ are present in a single image and therefore, we are able to detect even very small subunit exchange rates. Later, we will refer to the percentage of "exchanged" fluorescence and therefore exchanged subunits, as the "amount of exchanged subunits". 

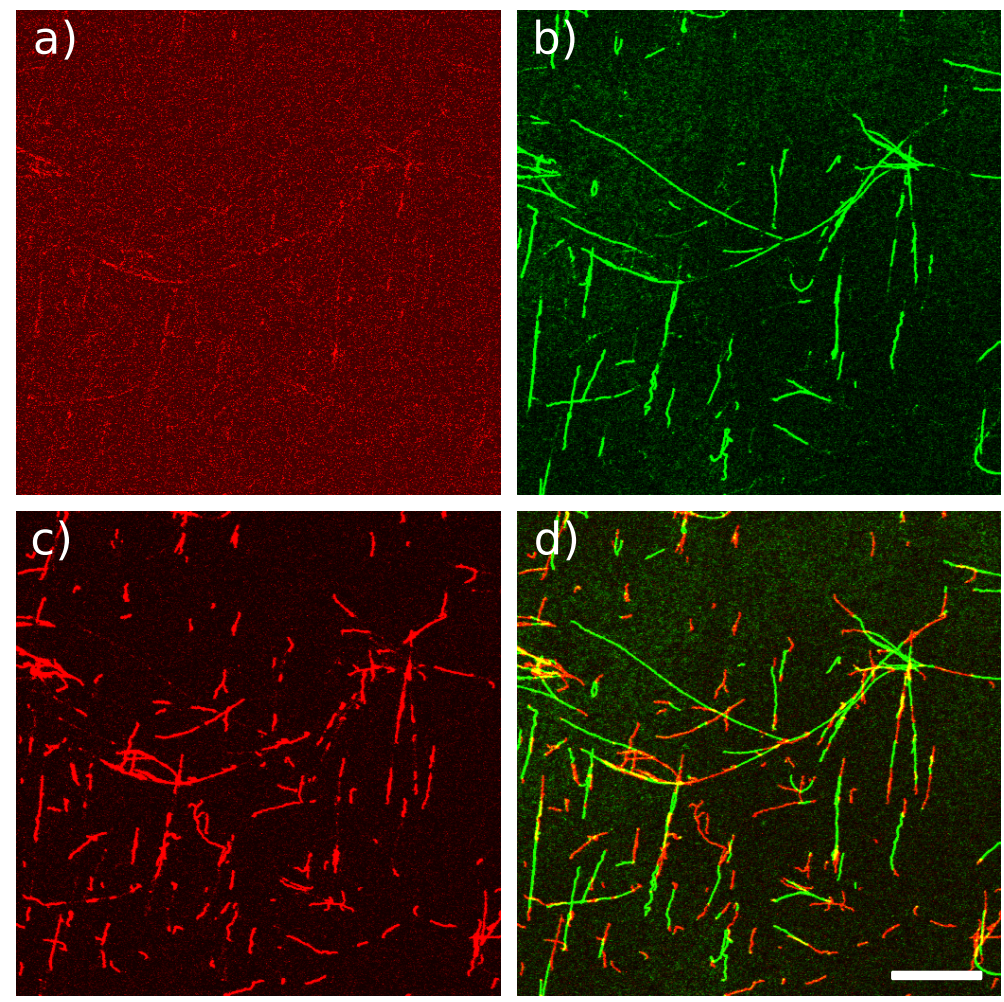

Figure 4.2: FRET measurements on vimentin intermediate filaments with red and green dyes. All images show the same sample region. a) FRET signal of the red dye is only observed when green dye is found at the same position. b) Green filaments define the possible region of FRET signal. c) Confocal microscopy image of red filaments acquired after the FRET measurement. d) Merge of c) and d). These images show that FRET measurements on our samples are possible. However, image quality of confocal microscopy images is preferable. Scale bar $20 \mu \mathrm{m}$.

\subsubsection{Control experiments}

As the fluorescence signal from exchanged subunits is not very strong, we have to take great care to exclude cross-talk between the two fluorescence channels we use. To this end, we perform extensive tests on samples with filaments labeled with both dyes we use. As the sample is static, the acquisition time for a single image can be freely chosen. Thus we can image the green and red channel sequentially, which already prevents cross-talk between channels to a large extent because the emission spectra of the dyes are well separated. However, we are nonetheless able to provoke cross-talk by using very high excitation laser intensities and thus have to carefully optimize imaging parameters to prevent cross-talk. In the end, we find that the imaging parameters we use lead to no detectable cross-talk. An example of these images is shown in figure 3.10 . 
To exclude that the subunit exchange we observe is an artifact associated with a specific dye, we also have performed experiments where the red dye is replaced by the green dye and vice versa. We find that the percentage of exchanged subunits is comparable to our "standard" setup. Consequently, all experiments are performed in this "standard" setup.

\subsubsection{Filament polymorphism}

As described in chapter 2.1, intermediate filaments can show polymorphism in their cross-section diameter, which is determined by the assembly process. Filaments with a uniform cross-section diameter are assembled in a dialysis bath [12. Filaments that show a high polymorphism of the cross-section are assembled by rapid dilution into assembly conditions. Filament polymorphism is thought to play a role in the exchange of subunits. Therefore, we compare the subunit exchange rates in different experiments on filaments which have either a uniform or a heterogeneous cross-section. Differences between filament populations with different polymorphism properties might allow us to draw conclusions regarding an exchange mechanism.

In the following we will refer to vimentin labeled with AlexaFlour 488 as "green" and to vimentin labeled with ATTO 647N as "red". In our experiments, we generally observe the exchange process from red subunits onto green filaments.

\subsection{Analysis}

To quantify the subunit exchange we use two different methods. We start with fluorescence images as shown in figure 4.3. In both cases a MatLab script is used to determine the positions of both red and green filaments. With this information it is now possible to compute a number of different values representing the fluorescence intensity in different regions of the image:

- The intensity of the red filaments $I_{f i l}$.

- The intensity in the red channel at the positions of green filaments $I_{e x}$. Regions where red and green filaments overlap are excluded from analysis.

- The intensity of the background in the red channel $I_{b a c k}$. The background is in this case defined as the absence of red as well as green filaments. 

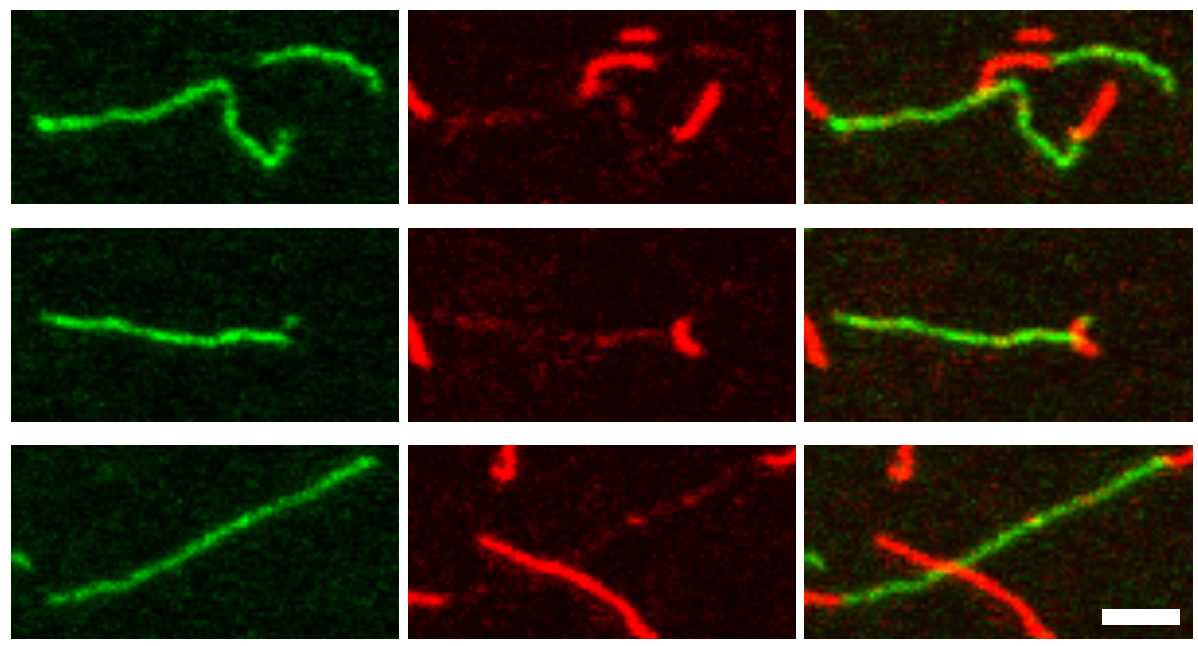

Figure 4.3: Fluorescence images of green and red vimentin after 300 minutes. (left) green channel (center) red channel (right) merge of red and green channels. Red fluorescence form exchanged subunits is detected on green filaments. Exchanged subunits are not homogeneously distributed and instead tend to form clusters. Scale bar $2 \mu \mathrm{m}$.

We use these values to calculate subunit exchange rates from red subunits into green filaments. We exclude the exchange of green subunits into red filaments from our analysis because red filaments in these experiments are mostly assembled in the presence of pre-assembled green filaments. Therefore, it would be unclear, if green subunits in red filaments are present because of a subunit exchange process, or simply because they are integrated in the filament during the assembly process.

\subsubsection{Percentage of exchanged subunits with red filaments as reference}

A straightforward method to calculate the relative amount of exchanged subunits is to use the value $I_{f i l}$ as reference for a filament consisting of, at least initially, $100 \%$ red subunits. This value is calculated for each individual image. We calculate the percentage of transferred subunits $A_{\text {sub }}$ with the equation

$$
A_{s u b, r}=\frac{I_{e x}-I_{b a c k}}{I_{f i l}-I_{b a c k}} .
$$

Here, we subtract the background intensity $I_{b a c k}$ from both the fluorescence intensity of the exchanged subunits $I_{e x}$ and the fluorescence intensity of the red filaments $I_{f i l}$. The subtraction of the background allows us to calculate the relative amount of exchanged subunits even in the very early stages of an experiment, where not yet 
assembled red vimentin contributes to the background intensity $I_{\text {back }}$. For ease of use we always refer to and plot percentages of exchanged subunits $A_{s u b}=A_{s u b, r} \cdot 100$.

Of course, the red filaments we use as a reference also can include green subunits, which is the "reverse" reaction of the subunit exchange process we observe. However, as the green vimentin is always used in filamentous form with very long assembly times, rates should be quite low. Also, the total percentage of transferred subunits remains quite low even after long times. Thus we assume that red filaments can be used as a reference for a standard filament throughout the whole experiment.

\subsubsection{Fluorescence intensity of subunits relative to red back- ground}

The second method we employ is independent of the value $I_{f i l}$. We basically calculate the the amount of exchanged subunits with respect to the background intensity. The equation we use is

$$
B_{\text {sub }}=\frac{I_{e x}}{I_{b a c k}} .
$$

Using this equation we can, of course, only compute the increase of subunit exchanged over time. A quantification of the subunit exchange process, as described above, is not possible with this method. However, we can use the value $B_{\text {sub }}$ to validate the findings we have obtained from our calculations of $A_{\text {sub }}$.

\subsubsection{Statistics}

We compute both values for the exchange of subunits, $A_{\text {sub }}$ and $B_{\text {sub }}$, for each individual image. Each image in turn contains several green filaments that contribute to $A_{\text {sub }}$ and $B_{\text {sub }}$. When we take samples every 60 minutes we typically acquire 15 images per time point that we can use for our analysis. Because the number of images included for each time point varies from experiment to experiment, we calculate the averages for both $A_{s u b}$ and $B_{s u b}$ for each single experiment. Thus we obtain curves characterizing the subunit exchange in a single experiment for up to 7 hours. We then calculate the mean value of all curves with the same experimental setting. The error we plot in our data is the standard deviation of the mean value. 


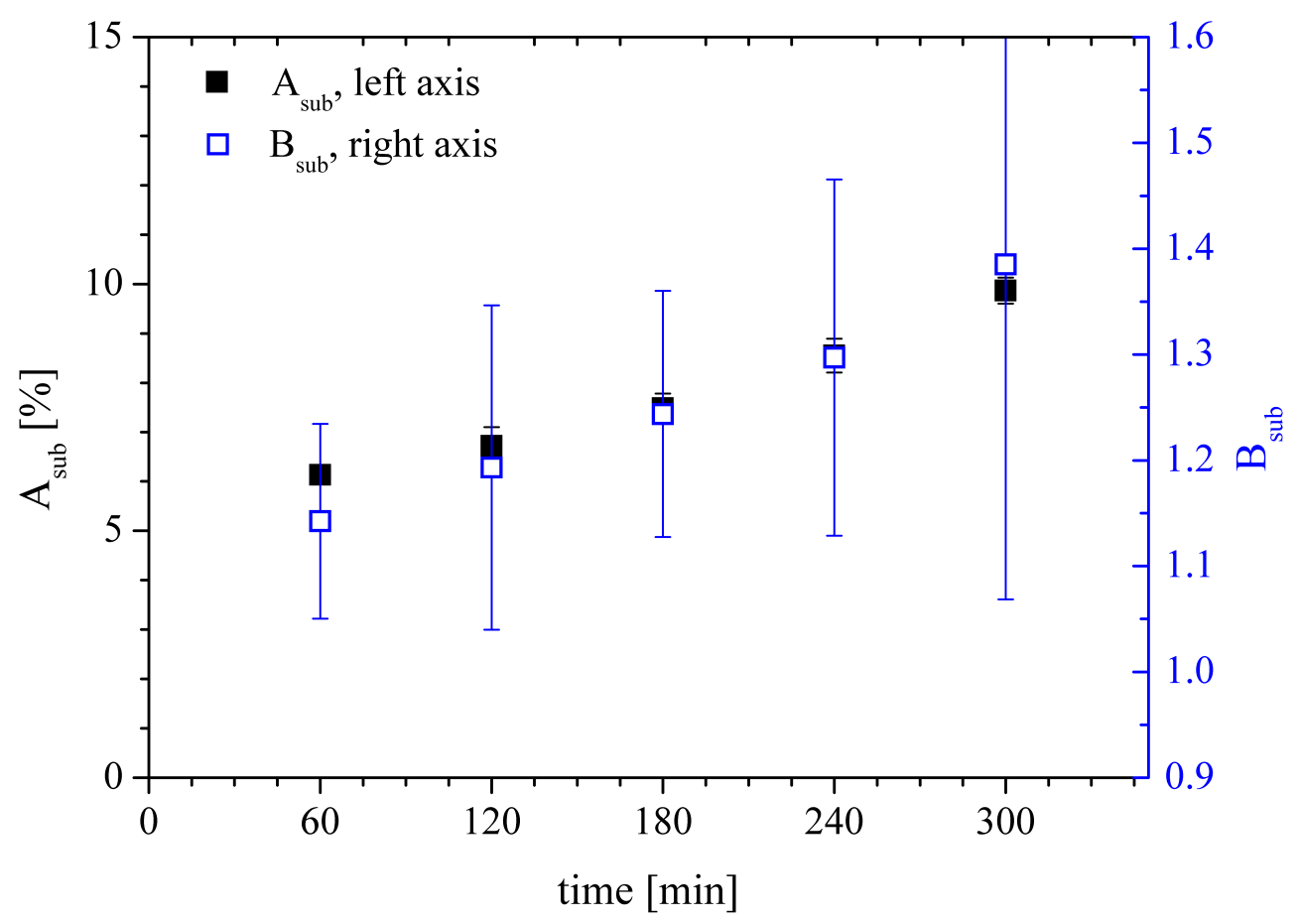

Figure 4.4: Two analysis methods yield similar curves. Only the calculations relative to filaments (solid symbols) give an absolute amount of exchanged subunits. By comparing with data from the second method (open symbols), which uses the background intensity as reference we can, however, confirm the general course of the subunit exchange process.

\subsection{Results}

With the tools described in chapter 4.2 we have computed the average subunit exchange over time for the different experimental conditions listed in chapter 3.1.5. We differentiate between filaments assembled in a dialysis bath and filaments assembled in a reaction tube. We have also examined the exchange of subunits at different temperatures.

A general observation we make, even before beginning with a detailed image analysis, is that the fluorescence of exchanged subunits is not homogeneously distributed over the filaments but rather forms clusters as shown in figure 4.3 . 


\subsubsection{Comparison of analysis methods}

We compare the two analysis methods we employ on a data set from filaments assembled in a reaction tube mixed with tetrameric vimentin. As shown in figure 4.4 the general properties of the subunit exchange process given by both analysis methods agree very well. We observe a relatively high offset of exchanged subunits at the first measurement time point (60 minutes). After the 60 minute mark we observe a slow increase of exchanged subunits.

The observation that both analysis methods yield similar results can be extended to all our other experiments. However, the measurement error is much smaller for the calculation of the relative amount of exchanged subunits. This, together with the straightforward interpretation of $A_{\text {sub }}$ makes the comparison with the filaments more attractive than the comparison with the image background. In the following, we will therefore only show the data for $A_{s u b}$ that gives the relative amount of exchanged subunits.

\subsubsection{Measurements with high sampling frequency}

In figure 4.5 we show two curves with a high sampling frequency. Each curve shows data from a single experimental day. An error is obtained from the standard deviation of the values $A_{s u b}$ of each individual image. To increase the sampling frequency, we have to reduce the number of images per time point. Therefore, statistics of these data sets are not optimal. However, we can nicely observe a steep increase of exchanged subunits in the first 60 minutes, which explains the offset of the curves in chapter 4.3.1. As the subunit exchange between mature filaments does not show this incline, it is very likely that the initially free tetrameric vimentin is integrating into the filaments.

In the following we focus on the increase of exchanged subunits after the 60 minute mark, where the initially free tetramers have assembled into filaments.

\subsubsection{Effects of different assembly methods and tempera- ture}

The results shown here are obtained from three distinct experiments. We observe the subunit exchange between mature red and green filaments assembled in dialysis tubing ("condition I"), between red tetramers and green filaments assembled in either dialysis tubing ("condition II") or a reaction tube ("condition III"). Plots 


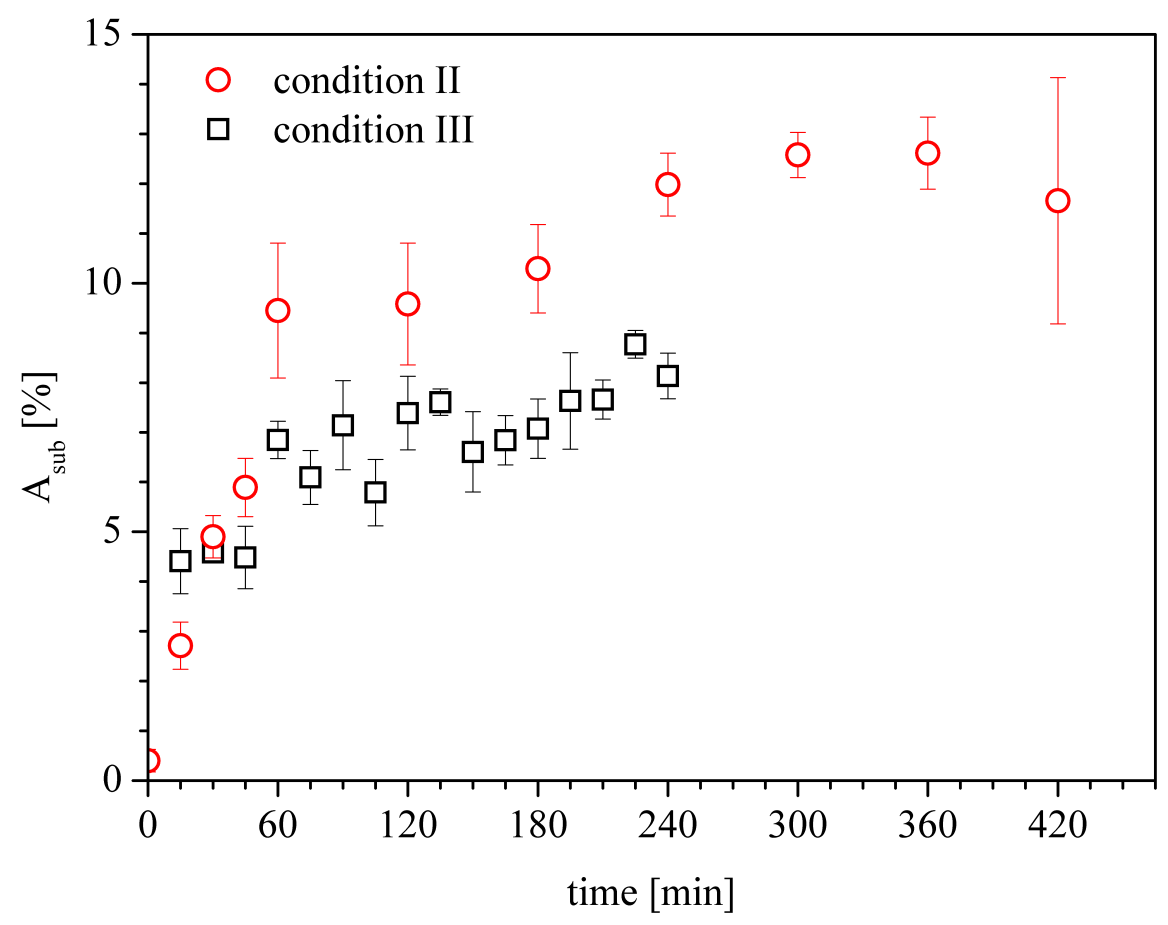

Figure 4.5: Subunit exchange observed with high sampling frequency. When samples are taken at a higher frequency at least for the first 60 minutes, we observe the faster initial subunit exchange, likely because more free tetramers are found in solution. The curves each show a single experiment with duration of 4 or 7 hours, respectively.

showing the percentage of exchanged subunits $A_{\text {sub }}$ over time for all three cases are given in figure 4.6. We observe that the addition of tetrameric vimentin to pre-assembled filaments results in a large amount of subunit exchange in the first 60 minutes and a slow exchange following the initial phase. Differences between dialysis assembled filaments and those assembled in a reaction tube are negligible. As expected, we do not observe a strong initial exchange of subunits for the differently labeled filaments of condition I.

Apart from different assembly methods we also compare identically prepared "combination III" samples at different temperatures. While one sample is kept at room temperature for the duration of the experiment a second sample is kept at $37^{\circ} \mathrm{C}$ as usual. The subunit exchange coefficient $A_{\text {sub }}$ is plotted for both cases in figure 4.7. For the lower temperature we see a similar general behavior to that of the sample kept at $37^{\circ} \mathrm{C}$. However, the initial exchange of subunits is reduced. Also, the exchange rate for the duration of the experiment is lower at a reduced temperature. 


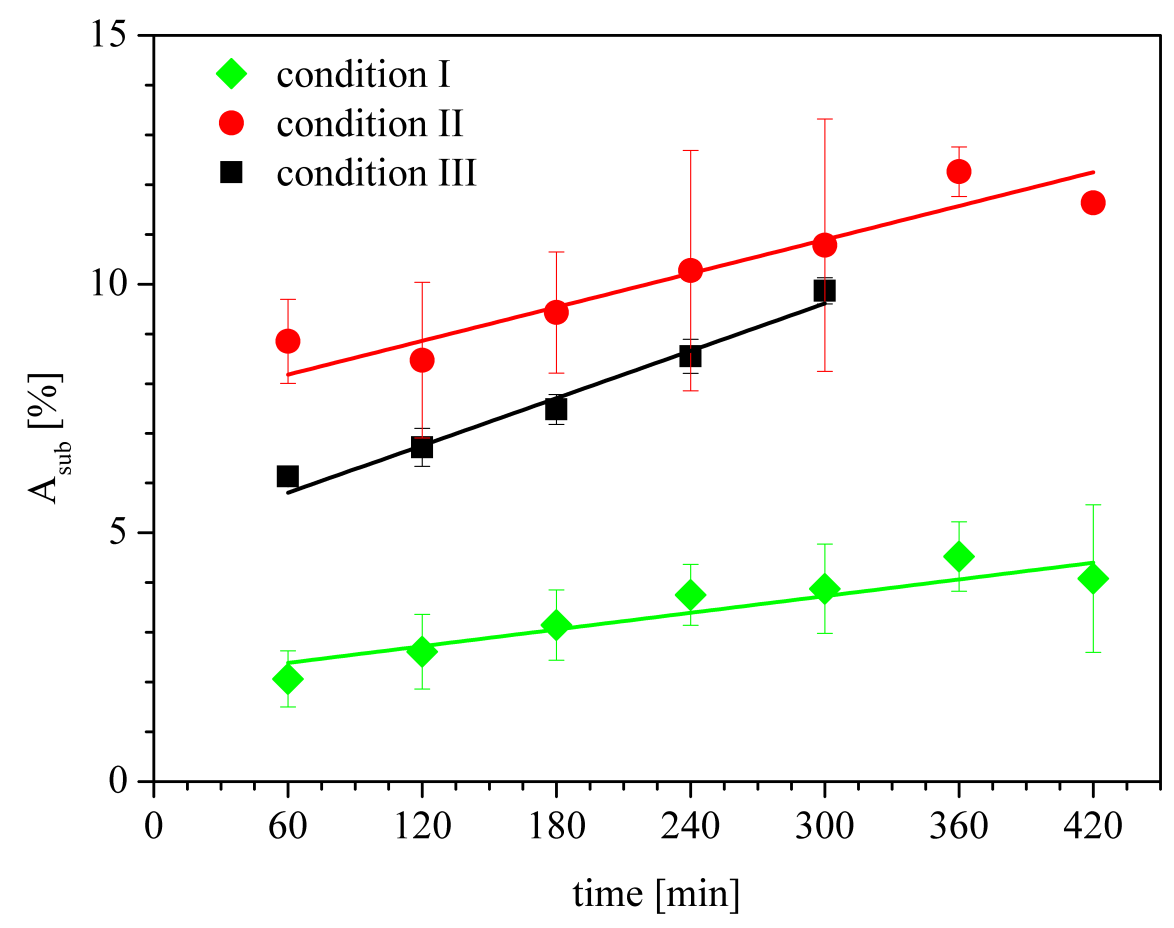

Figure 4.6: Comparison of different experimental parameters. Overall, subunit exchange is more pronounced when we combine tetrameric vimentin with filaments. However, the differences between conditions II and III are very small - subunit exchange is very similar regardless of assembly method. Lines show linear fits for the individual curves, slopes are listed in table 4.1 .

As the initial subunit exchange is finished before the 60 minute mark, we can estimate an average subunit transfer per time through a linear fit of the individual curves. Here, we use a linear fit, because it is the most straightforward assumption we can make. Also, the percentage of exchanged subunits remains low even for long observation times and a strong deviation from a linear exchange process is only expected for a higher exchange percentage. Additionally, a linear fit describes our data very well and therefore a more elaborate fitting function is not necessary.

Notably, we do not expect a line through the origin because of initial exchange. The values for the subunit transfer slope are given in table 4.1. As expected, the values at $37^{\circ} \mathrm{C}$ are lowest for "condition I" where we combine mature filaments. Values for "condition II" are similar to those for "condition III". Lowering the sample temperature leads to a strongly reduced subunit exchange for "condition III". For "conditions I \& II" we expect a similar reduction of the exchange rate at lower 


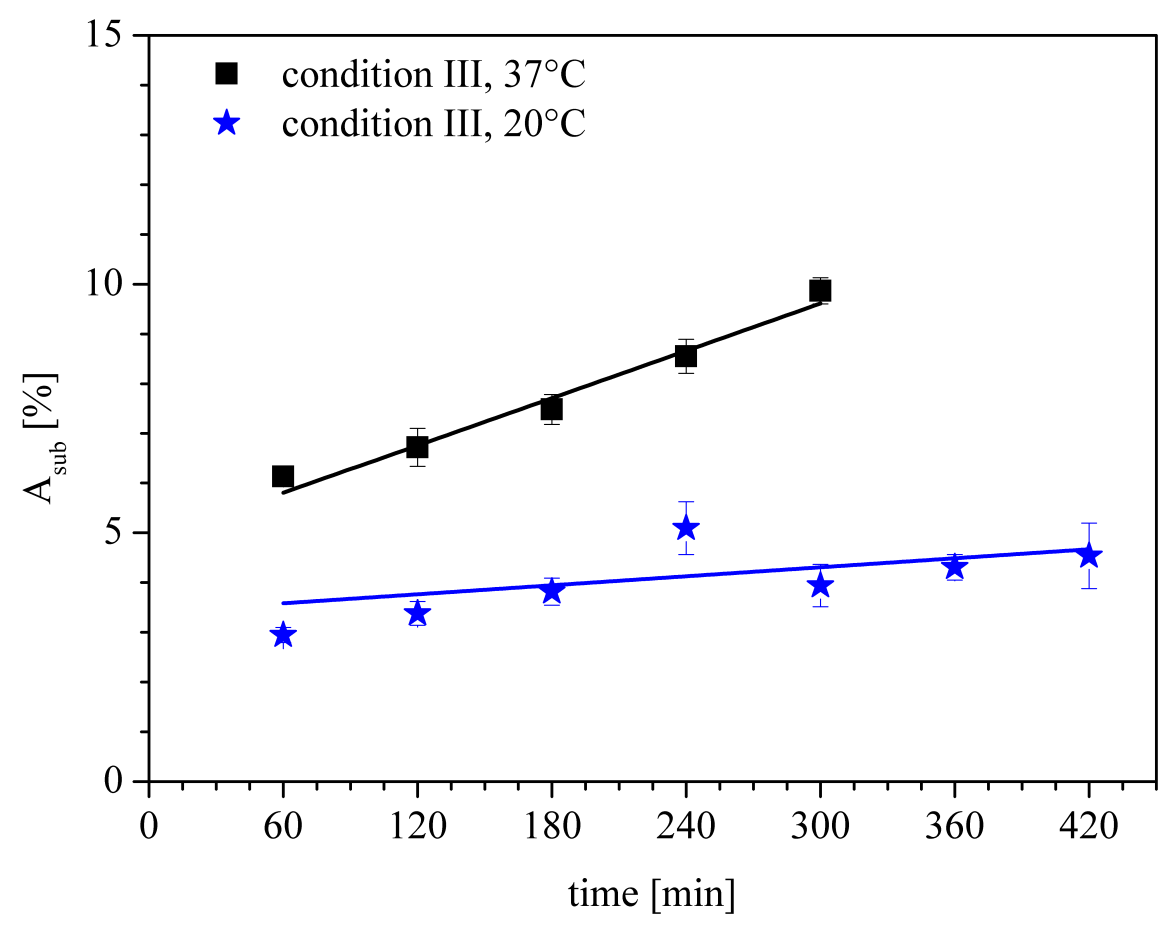

Figure 4.7: Subunit exchange is temperature dependent. Two experiments with identical starting conditions but different incubation temperatures after the addition of tetrameric vimentin show a disparate subunit exchange rate. The initial subunit exchange as well as the subunit exchange rate over several hours is lower for reduced temperatures.

temperatures.

\begin{tabular}{l||l} 
Setup & Exchange coefficient $[\% / \mathrm{h}]$ \\
\hline \hline condition I & $0.34 \pm 0.06$ \\
condition II & $0.68 \pm 0.10$ \\
condition III, $37^{\circ} \mathrm{C}$ & $0.95 \pm 0.09$ \\
condition III, $20^{\circ} \mathrm{C}$ & $0.18 \pm 0.12$
\end{tabular}

Table 4.1: Subunit exchange coefficients for different experimental setups.

\subsubsection{End-to-end annealing}

Additionally to the measurements of the subunit exchange rate, we also observe the end-to-end annealing of vimentin intermediate filaments. Clear evidence of the 

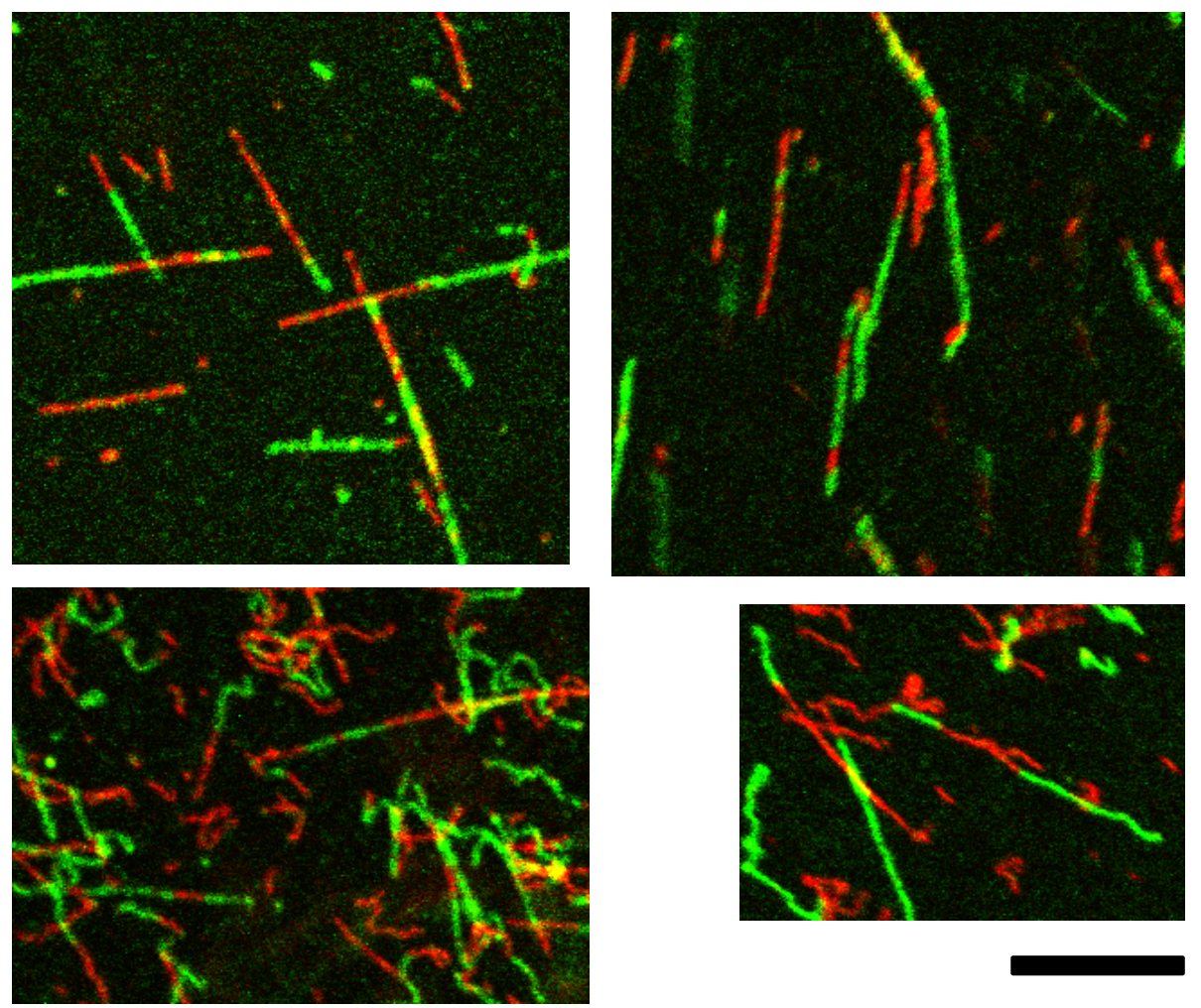

Figure 4.8: Vimentin intermediate filaments anneal end-to-end. An inclusion of a red filament segment between two green filament segments can only occur, when filaments anneal end-to-end. Scale bar $10 \mu \mathrm{m}$.

end-to-end annealing is the inclusion of a red filament section between two green filament sections as shown in figure 4.8. The pre-existing green filaments can only include a red section by end-to-end annealing.

We observe the end-to-end annealing process for filaments assembled in dialysis tubing as well as in a reaction tube. In the images we have acquired for the measurement of the subunit exchange process, end-to-end annealing events are not very frequent. Together with the fact that our images were acquired for the analysis of the subunit exchange reaction, we can only give a very rough estimate. Where we find that about $1 \%$ of all filaments are a product of end-to-end annealing. Here, we of course we can not detect filaments that are wholly green or consist of a single red and a single green part and are nonetheless the product of an end-to-end annealing process.

With the knowledge that end-to-end annealing is possible for vimentin filaments, we can also determine if filaments (frequently) break in the middle followed by end-to-end annealing in an equilibrium reaction. When we consider two populations of filaments, one red and one green, with a specific length distribution at the 
beginning of the experiment we would make the following observations: If filaments break frequently, and bind to other filaments by end-to-end annealing, the average length of a filament section of uniform color shrinks over time. For example, a filament consisting initially of a long red and a long green part changes, given enough time, into a filament with many short, alternating red and green stripes. As we do not observe a reduction of the "stripe length" in our experiments, it is unlikely that vimentin filaments frequently break apart when they are in assembly buffer. Also, the low end-to-end annealing rate we observe, shows that frequent breaking and annealing are not in evidence.

\subsection{Discussion}

It is our aim to describe the subunit exchange in vimentin intermediate filaments in vitro. The results we have shown in chapter 4.3 give us enxtensive insights into this reaction. The general course of the different subunit exchange curves describes an increase over time with a strong increase at the beginning of an experiment and a linear increase in exchanged subunits thereafter.

\subsubsection{The first 60 minutes of an experiment}

The subunits exchange in the first 60 minutes of an experiment proceeds very fast in comparison to later stages. The reason for this is the large number of free tetramers found in solution at the beginning of an experiment of either condition II or III. On the one hand, these tetramers rapidly begin to assemble into filaments. On the other hand, some of these tetramers (or larger aggregates) integrate into existing filaments and thus fill vacant "slots" in a subunit exchange process. Alternately, filament polymorphism may cause subunits to attach to an existing filament without filling an empty "slot" and thereby expand the filaments cross-section locally. After a certain time, the initial subunit exchange process begins to slow down. At this time most tetramers and other low assembly states have been assembled or integrated into filaments. According to our measurements most tetramers are incorporated into filaments after 60 minutes. This value is also supported by assembly experiments in diffusion chambers as shown in chapter 5.2.2. Furthermore, controls of vimentin assembly with electron microscopy as well as theoretical models predict that after 60 minutes virtually all protein is incorporated into filaments 10,75 . Consequently, after 60 minutes the amount of free subunits should be very similar in all three cases, 
conditions I-III. Differences between the different experimental conditions lie now mostly in the assembly mode of the pre-existing filaments. Filaments assembled in a dialysis bath show a very similar overall exchange of subunits as filaments assembled in a reaction tube, at least when combined with tetrameric vimentin. This indicates that the assembly method does not play a significant role in the subunit exchange process when ample free subunits are present. Filament polymorphism at the start point is negligible for this question when tetrameric vimentin is added to filaments.

If we now consider the case of two differently labeled dialysis assembled filaments in "condition I", the overall percentage of exchanged subunits is notably lower than for the addition of tetramers. This observation correlates with the lower amount of free tetrameric vimentin in the first 60 minutes that we expect because of the already long assembly times.

\subsubsection{Linear exchange of subunits}

After the first 60 minutes of an experiment, the subunit exchange rate remains at a relatively constant value, so that we use a linear fit to determine this rate. If we limit our discussion to the experiments performed at $37^{\circ} \mathrm{C}$, we find the following: The exchange rate for only filaments in condition $\mathrm{I}$ is lower than for the case of tetramer addition in both conditions II and III, where we find comparable exchange rates. The reduced transfer rate for condition I can be explained by the reduced polymorphism of the dialysis assembled filaments. While the incorporation of subunits into these filaments is the same as for filaments assembled in a reaction tube, the release of subunits into the soluble pool likely proceeds at a lower frequency. We can make this assumption because the average cross-section of dialysis assembled filaments contains a lower number of monomers than the cross-section of filaments assembled by rapid dilution. Herrmann et al. find an average number of 29 monomers per crosssection for dialysis assembled filaments and an average number of 36 monomers per cross-section for filaments assembled through dilution into assembly conditions [12].

The different subunit exchange rates found with different experimental parameters also show very nicely that the exchange of fluorophores we observe, is in fact an exchange of subunits. An exchange of only fluorophores, without an accompanying subunit exchange, would not show different exchange rates for different experimental parameters.

As we have discussed in chapter 4.4.1, the conditions in an experiment after the first 60 minutes depend mostly on the assembly mode of the filaments and therefore 
their cross-section polymorphism. The percentage of exchanged subunits proceeds in a linear fashion and only the offset from the initial subunit exchange process remains. Consequently, we can interpret the later stages of condition II as a separate experiment, where filaments assembled in both dialysis bath and reaction tube are present. The later stages of condition III give results for the case of two differently labeled filaments, which are assembled in a dialysis bath. The differences in subunit exchange rates between the two experiments condition II and III are very small, this observation strengthens the argument we have made above: filaments assembled in a dialysis bath with a uniform, small cross-section can "accept" subunits but seldom release them. Whereas filaments assembled in a reaction tube have a heterogeneous cross-section and "accept" as well as release subunits.

Apart from the influence of the filaments' polymorphism, we have found that subunit exchange rates decrease at $20^{\circ} \mathrm{C}$. Because we assume that a temperature change has an effect independent of filament polymorphism, we use the experimental parameters with the highest exchange rate, condition III. Here, we lower only the sample temperature, while all other parameters remain unchanged. We find that the experiment with the lowest overall subunit exchange rate is that performed at $20^{\circ} \mathrm{C}$. The lowered temperature leads to a strongly reduced exchange rate of $0.18 \% / \mathrm{h}$ in contrast to an exchange rate of $0.95 \% / \mathrm{h}$ at $37^{\circ} \mathrm{C}$. A reduction of the exchange rate is, at least in part, a consequence of the temperature dependence of the diffusion process discussed in chapter 4.4.3.

\subsubsection{Diffusion of subunits is relevant for the exchange re- action}

The exchange of subunits between filaments is only possible because subunits move through diffusion. At least part of the reason for the reduced subunit exchange rates at lower temperatures is the following: diffusion is a temperature dependent process. However, diffusion at $20^{\circ} \mathrm{C}$ still carries on at $95 \%$ of the original rate compared to the case at $37^{\circ} \mathrm{C}$, when we assume a linear dependency on temperature of the diffusion coefficient as shown in equation 5.2. However, the diffusion coefficient is also influenced by the viscosity of the bulk material, which is in our case an aqueous solution. While the viscosity of water at $20^{\circ} \mathrm{C}$ is $1 \mathrm{mPa} \cdot \mathrm{s}$, the viscosity at $37^{\circ} \mathrm{C}$ is calculated to $0.69 \mathrm{mPa} \cdot \mathrm{s}$ [76. Taken together the influences of the lowered temperature result in a still lower diffusion coefficient. In numbers, the diffusion coefficient for tetrameric vimentin at $20^{\circ} \mathrm{C}$ is $D_{t}=2.4 \times 10^{-11} \mathrm{~m}^{2} / \mathrm{s}$ as calculated in chapter 


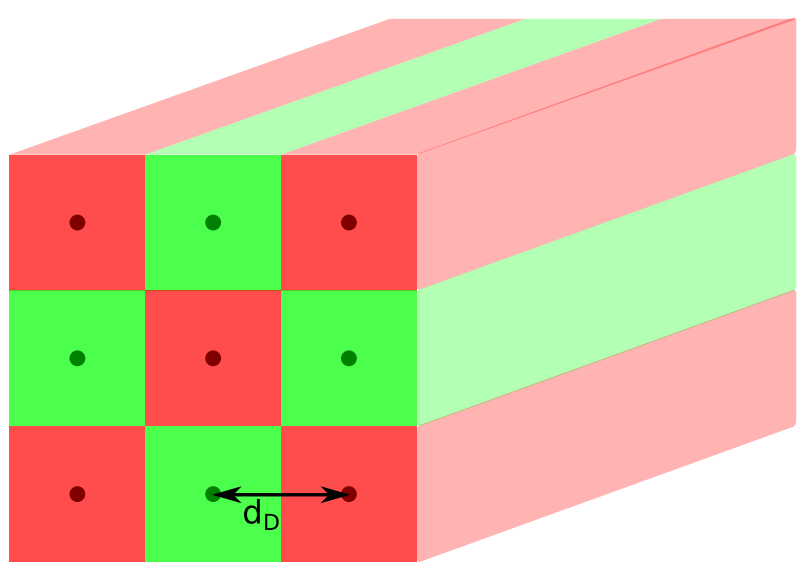

Figure 4.9: Illustration of the diffusion distance $d_{D}$. If two filament types occupy a square region along their contour, the average diffusion distance between the filaments is $d_{D}$.

5.1 and the diffusion coefficient at $37^{\circ} \mathrm{C}$ is calculated to $D_{t, 37^{\circ} \mathrm{C}}=1.6 \times 10^{-11} \mathrm{~m}^{2} / \mathrm{s}$. However, the difference in diffusion speed between the two different temperatures only accounts for about half of the total difference in the subunit exchange rates at different temperatures. A reason for the further reduction in subunit exchange rate could be an overall slowdown of the exchange reaction itself - the probability for a subunit to attach or detach from a filament has to be reduced at lower temperatures. However, measurements supporting this assumption are largely missing.

The time it takes a subunit to move from a "donor" filament to an "acceptor" filament through diffusion depends not only on the diffusion coefficient but also on the average distance between the filaments. Because the total protein concentration is very similar in all our experiments at $c \approx 0.2 \mathrm{~g} / \mathrm{L}$, the average distance is similar for conditions I to III. To calculate the average distance between the filaments, we perform a calculation in analogy to a face centered cubic crystal lattice, found for example in sodium chloride. However, the filaments are hardly point-like. We thus modify the lattice as illustrated in figure 4.9, where each filament is assigned a volume represented by a square cuboid and the square cross-section of this cuboid is denoted $A_{F}$. If we assume that the filaments are perfectly straight, we can calculate the area $A_{F}$ for filaments in a homogeneous solution with concentration $c$ with the equation $A_{F}=\frac{M_{L}}{c}$. Here, $M_{L}$ is the mass per length of vimentin filaments. If we now assume we have two filament types with different colors, as illustrated in figure 4.9 , we can estimate the average distance between two neighboring filaments 
of different color to

$$
d_{D}=\left(\frac{M_{L}}{c}\right)^{\frac{1}{2}} .
$$

In this case, $c$ is the total concentration of both filament types. We can calculate an average distance between a red and a green filament of $d_{D} \approx 0.3 \mu \mathrm{m}$ for a solution with a total vimentin concentration of $0.2 \mathrm{~g} / \mathrm{L}$. With the average distance between filaments, we can estimate the timescales it takes a subunit to move from the "donor" filament to an "acceptor" filament. With a diffusion coefficient for vimentin tetramers of about $D_{t}=2.4 \times 10^{-11} \mathrm{~m}^{2} / \mathrm{s}$ as calculated in chapter 5.1 and the assumption of a radial travel distance of $1 \mu \mathrm{m}$ until the tetramer encounters a filament, we obtain a travel time of $T_{t} \approx 10 \mathrm{~ns}$. The model we have described above uses well aligned straight filaments. In reality, the filaments are flexible and arranged in a random pattern. Consequently, the average distance between the filaments is likely smaller than the value we have calculated. This also means that the timescales of the diffusion process we have calculated are the upper limit. The above considerations, together with the highest rate of the subunit exchange we observe, which is about $1 \%$ per hour, allow us to determine the concentration of free subunits. In this estimation we only use the exchange rate and the travel time and disregard the actual binding and unbinding reactions. We calculate the amount of free subunits that are in the sample to $5 \times 10^{-15} \mathrm{~g} / \mathrm{L}$ at any given moment. This corresponds to about 14000 free tetramers in one milliliter of sample. In contrast to this, the total amount of protein in filamentous form consists of about $10^{17}$ tetramers. The very low number of free tetramers agrees quite well with the observation that in a solution with filaments assembled for a long time, basically no free protein is observed.

\subsubsection{Comparison with literature}

The exchange of subunits in intermediate filaments has been first discussed by John Ngai at the beginning of the 1990's [20]. Further experiments were conducted thereafter 21,77]. However, detailed measurements, in the cell or in vitro were largely missing until recently. A study by Çolakoğlu and Brown on the subunit exchange of vimentin filaments in SW13 cells gives quantitative data that we can compare to our measurements [17]. The authors use dual colored vimentin tagged with either GFP or mCherry fluorescent proteins and thus use the same basic principle to detect the subunit exchange as we do. By fusing cells with vimentin tagged with GFP 
and mCherry, respectively, Çolakoğlu and Brown combine two differently labeled vimentin populations. Notable differences between our experiments exist, however, in the fluorescence label. The molecular weight of the fluorescent proteins is about 25 times as high as that of the synthetic dyes used in our experiments. A second, more relevant difference is that our experiment is by design not influenced by cellular regulatory mechanisms, whereas those mechanisms are naturally present in a cellular system.

Çolakoğlu and Brown find an increase of red fluorescence on green filaments after 8 hours that is quantitatively similar to the exchange of subunits observed by us after 7 hours. The overall subunit exchange thus proceeds at similar speeds in the cell and in vitro. This similarity between cellular system and in vitro experiments indicates that the subunit exchange in vimentin intermediate filaments is an inherent property of the polymer system and not exclusively managed by cellular mechanisms.

\subsubsection{Implications of subunit exchange}

The exchange of subunits could have different implications for the properties of the filament and therefore, in the end, the cytoskeleton of the cell as a whole. The polymorphism shown by the intermediate filaments most likely leads to different mechanical properties of the filament. Thus, incorporation or loss of subunits in the filament could lead to changed mechanical properties. This process could be directed by locally increasing or decreasing the amount of subunits in the soluble pool or through regulation of protein-kinase activity.

Also, the subunit exchange mechanism could be relevant for the differentiation process of the cell. In many cases of cell differentiation the intermediate filament network rearranges or the type of intermediate filament changes altogether [78 80]. For instance in the development of skeletal muscle cells the composition of the intermediate filament network changes from mainly vimentin and nestin to desmin as the primary intermediate filament [78]. Interestingly, both vimentin and nestin and vimentin and desmin co-assemble into heteropolymers under certain conditions 81 83. It could be possible that the exchange of intermediate filament subunits plays a role in the change from one intermediate filament system to another, when the different involved polymers can co-assemble into filaments. However, further experiments are necessary to test this hypothesis. 


\subsubsection{End-to-end annealing and filament stability}

The end-to-end assembly mechanism has been found to be necessary to establish a model for the assembly of vimentin filaments as discussed in references [10,15]. This assembly mode was first observed in vitro in a work of Winheim et al. in 2011 [16. Observations in the cell have been made prior to these measurements [17. The end-to-end annealing process thus complements the filament assembly by the addition of a single unit-length filament. As we have observed end-to-end annealing in filaments assembled in a dialysis bath as well as a reaction tube, we can assume that this assembly path is independent from filament polymorphism.

In all cases we have thus far considered, the exchange of subunits does not lead to an obvious instability of the filament. The pre-assembled green filaments do not change in length over many hours. This means that the filaments do not break apart with a high frequency during our experiments. To investigate the possibility of filaments breaking in the middle, we perform disassembly experiments by reducing $\mathrm{KCl}$ concentration. These experiments are described in detail in chapter 5.2.3. Still, stable filaments give further information about the subunit exchange process. Exchanged subunits are limited in size to most likely tetrameric or octameric vimentin, because larger "subunits" would likely lead to a break in the filament. However, further experiments are necessary to determine the exact properties of subunit. 


\section{Chapter 5}

\section{Diffusion chambers}

By employing microfluidic-diffusion-chambers, it is our intention to observe the exchange of vimentin filament subunits as already described in chapter 4 in situ and with a high time resolution. In the scope of these experiments, we also employ diffusion-chambers to characterize the assembly and disassembly process of vimentin filaments. Here, our second focus is the question, if the filaments break in the middle during the disassembly process initiated by the reduction of the $\mathrm{KCl}$ concentration or if they simply fall apart from the filaments ends. Therefore, we aim to assemble filaments where the center part is fluorescently labeled with one dye and both tips are labeled with a different dye. Breaking filaments should consequently show an exposed end labeled with the "center" dye. To this end, we begin by characterizing the diffusion chambers we use with simulations done in Comsol 4.4 (Comsol Multiphysics, Göttingen, Germany).

\subsection{Characterization of the device}

With the help of simulations, we can characterize the diffusion chambers introduced in chapter 3.3.2. The main points of interest to us are the characteristic timescale for the diffusion of salt ions on the one hand and vimentin on the other hand. When we consider these timescales, we always refer to the time it takes to reach equal concentrations of salt or vimentin in both channel and chamber. The typical diffusion timescales are important for our experiments, because with their knowledge we can estimate the concentrations of the reactants (vimentin or $\mathrm{KCl}$ ) in the chambers. In the case of vimentin, we need to make certain that the timescales for the diffusion process into the chamber are compatible with our experiment. Mostly, this means 




Figure 5.1: Diffusion chambers with fluorescent beads. The fluorescent beads in the buffer solution are used to visualize the flow in the diffusion-chamber-device by using long exposure times. As a consequence of a to large inlet in the middle chamber the flow reaches into the chamber, which is shown by the bead streaks. Scale bar $20 \mu \mathrm{m}$

that the inflow time should not be exceedingly long. However, we also need to avoid very short inflow times for vimentin, since in this case, the protein can diffuse out of the chamber in a reverse process even before the assembly process can be initialized and the larger vimentin aggregates are unlikely to leave the chamber by diffusion because of their mechanical stiffness, discussed in chapter 6. In general, we have to consider that times for diffusion in and out of the chamber are equal:

$$
T(\text { inflow })=T(\text { outflow })
$$

The timescales for diffusion from the main channel into the actual chamber can be influenced by two factors. The dimensions of the inlet channel, where in general a short and wide channel leads to a fast diffusion process and a long and narrow channel results in longer diffusion times. Also, the size of the chamber itself plays a role. Large chambers are slower to equilibrate than small chambers. Thus, we can produce a microfluidic device with parameters such that we obtain timescales suitable for our purposes. To actually perform simulations of the diffusion-chamberdevices, we need the diffusion coefficients for both $\mathrm{KCl}$ and vimentin. For $\mathrm{KCl}$ we use the diffusion coefficient $D_{K C l}=1.9 \times 10^{-9} \mathrm{~m}^{2} / \mathrm{s}[84]$. In the case of vimentin, 


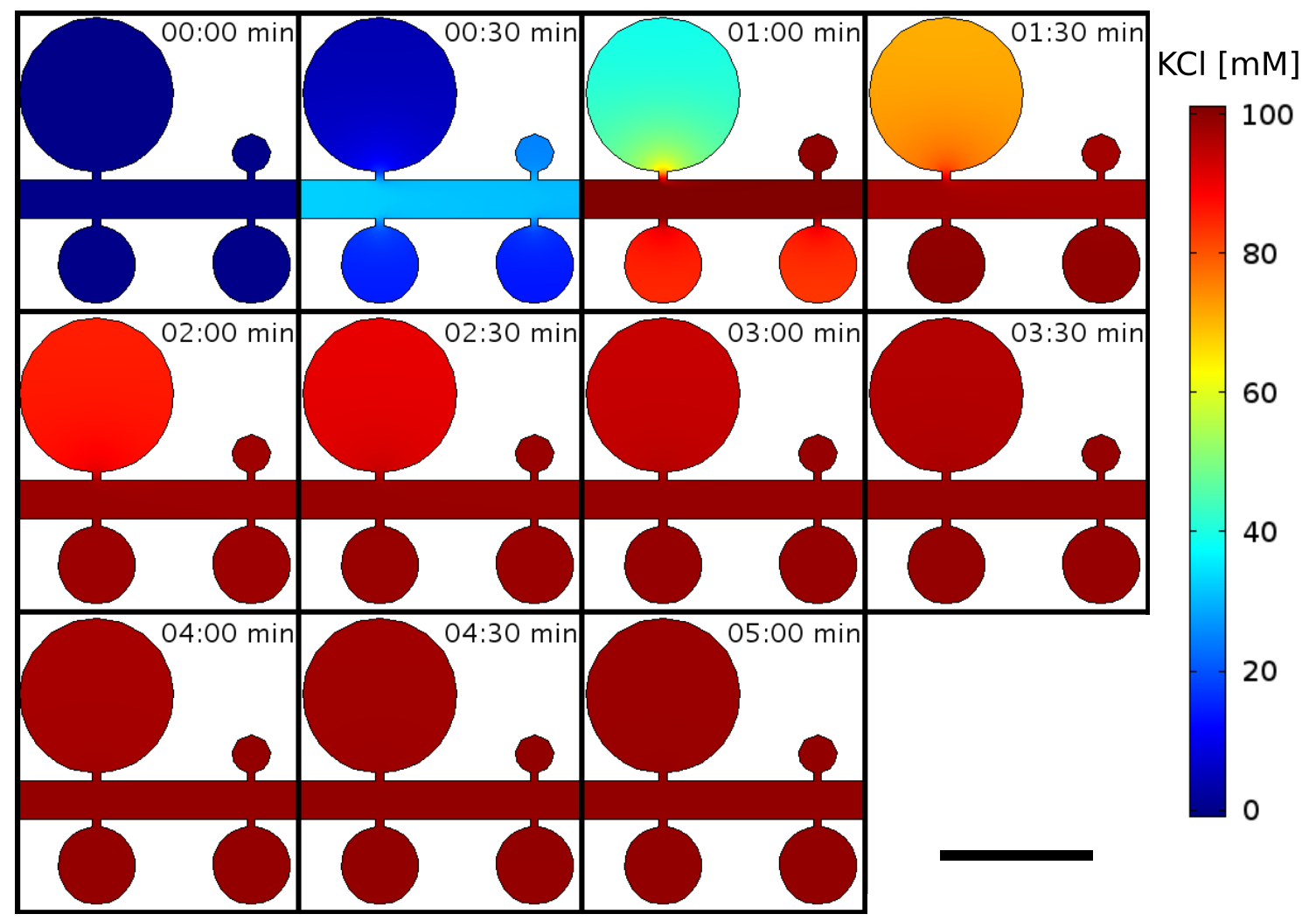

Figure 5.2: Simulation of $\mathbf{K C l}$ diffusion into chambers. The time series of the diffusion process shows that the $\mathrm{KCl}$ concentration in the device equilibrates within 5 minutes for the largest chambers. Diffusion times for the smaller chambers are again much lower, enabling us to choose between different timescales in one single device. Scale bar $200 \mu \mathrm{m}$.

we use the diffusion coefficient for the tetrameric form, which is flushed through the main channel and then needs to enter the chambers by diffusion. We estimate the diffusion coefficient for movement in a random direction from the size of the tetramer. For the calculation of the diffusion coefficient for a cylinder we use the following equation [85:

$$
D=\frac{\ln (p)+\nu}{3 \pi \eta_{0} L_{c}} \cdot k_{B} T .
$$

Here, $\eta_{0}$ is the viscosity of the buffer. The form factor $p=L_{c} / d_{c}$ indicates the aspect ratio of the "cylinder", where $L_{c}$ is the (tetramers) length and $d_{c}$ the diameter. For $\nu$ we use the expression $\nu=0.312+0.565 / p-0.100 / p^{2}$, which was obtained by fitting of numerical data [85]. In the case of vimentin, we estimate $p=60 \mathrm{~nm} / 3.4 \mathrm{~nm}=17.6[86$ and thus obtain an isotropic diffusion coefficient for tetrameric vimentin of $D_{t}=2.4 \times 10^{-11} \mathrm{~m}^{2} / \mathrm{s}$. For the purpose of comparison, it is also possible to calculate the values for anisotropic diffusion. Here, we obtain two 

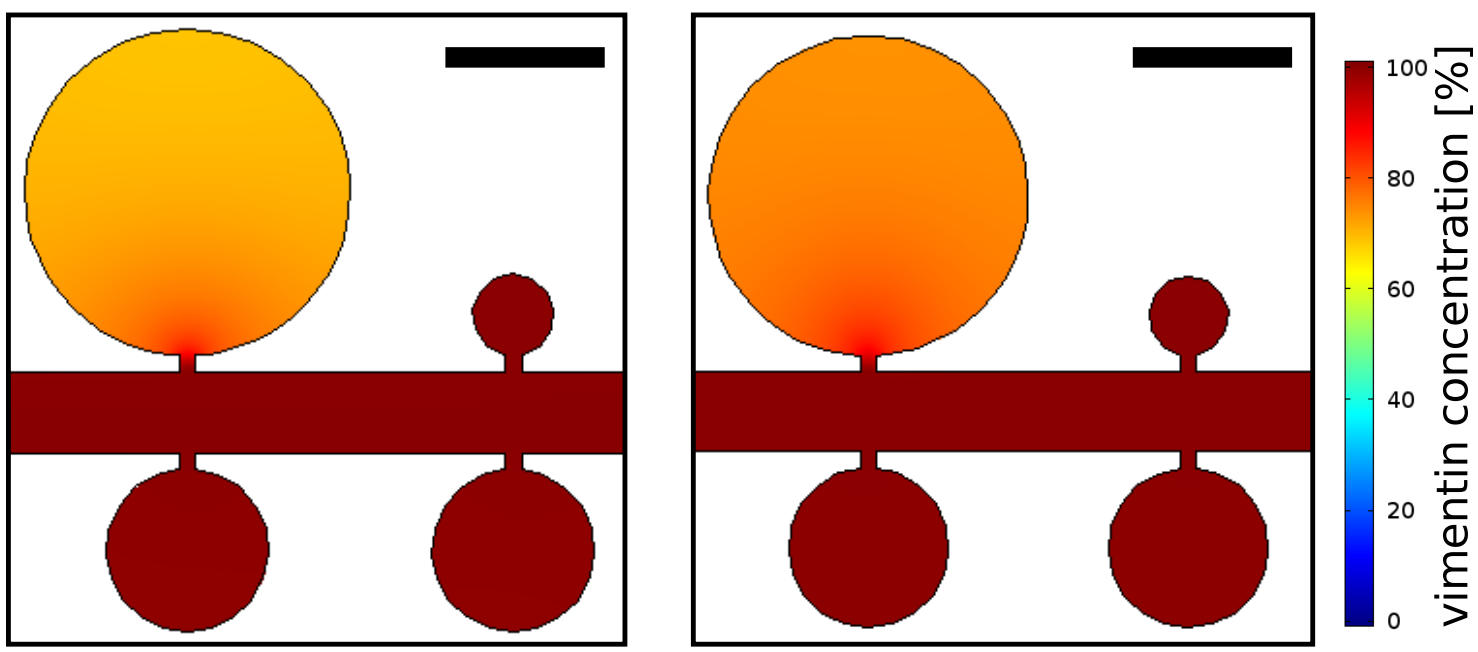

Figure 5.3: Comparison of isotropic and anisotropic diffusion of vimentin tetramers. Concentration of vimentin in diffusion chambers after 60 minutes when using an isotropic model (left) or an anisotropic model (right). Differences between the two models are small. Nonetheless, the anisotropic simulation shows a slightly faster equilibration process. Scale bar $100 \mu \mathrm{m}$.

values when using equations from reference [85], one for the diffusion parallel to the long axis $D_{t}^{\|}=3.0 \times 10^{-11} \mathrm{~m}^{2} / \mathrm{s}$ and one value for the diffusion perpendicular to this axis $D_{t}^{\perp}=2.0 \times 10^{-11} \mathrm{~m}^{2} / \mathrm{s}$. The use of an anisotropic diffusion coefficient results in a more realistic diffusion behavior in the simulation, because it takes into account the different behavior for diffusion in the direction of the long axis of the cylinder and perpendicular to it.

Experimentally, we obtained a good estimate of the diffusion coefficient of tetrameric vimentin by performing fluorescence correlation spectrometry (FCS) experiments in the laboratory of Prof. Detlev Schild. We have measured a diffusion coefficient of $D_{t, \text { exp }}=(2.6 \pm 0.2) \times 10^{-11} \mathrm{~m}^{2} / \mathrm{s}$. This value is very similar to the value we calculated from the dimensions of the protein $D_{t}=2.4 \times 10^{-11} \mathrm{~m}^{2} / \mathrm{s}$.

Apart from the diffusion coefficients of the samples, the parameters for the simulation depend also on the flow speed in the channel. As shown in figure 5.1, high flow rates have to be avoided to prevent flow into and in the diffusion chambers. The possibility of flow in the chambers is also imposing a lower limit on the length of the inlet to the chambers. Choosing an inlet with a length of at least $10 \mu \mathrm{m}$ and a flow rate no higher than $50 \mu \mathrm{l} / \mathrm{h}$ prevents these complications effectively. Also, we perform simulations only in a 2 dimensional system because diffusive processes can be nicely described by a 2 dimensional equation. The velocity field of a slow fluid flow in a 2 dimensional simulation is a very good approximation of the real flow field. 


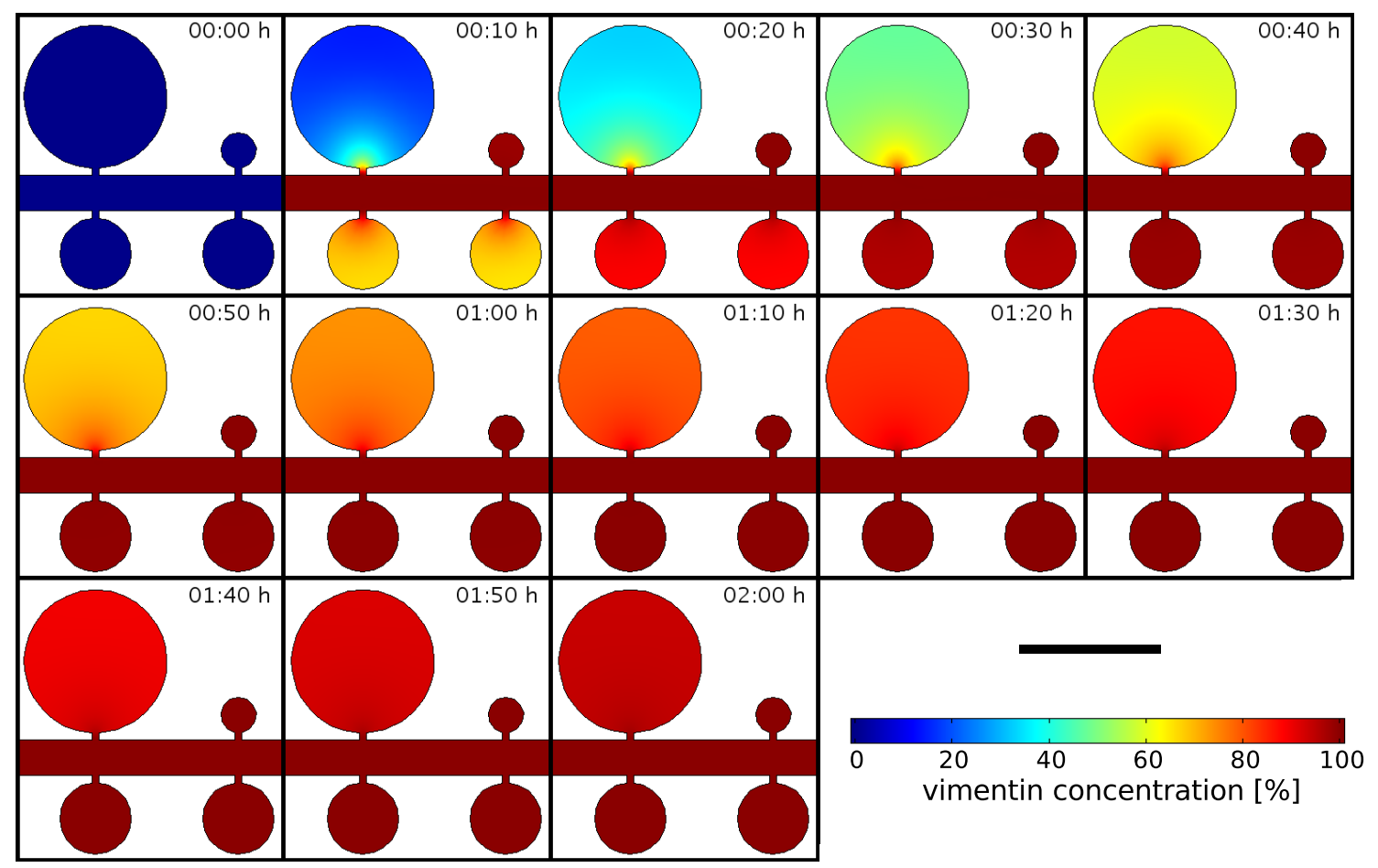

Figure 5.4: Time series of the anisotropic diffusion of vimentin tetramers. Diffusion of tetrameric vimentin into chambers with a diameter of $200 \mu \mathrm{m}$ occurs on the timescale of 120 minutes. All smaller chambers are equilibrated after approximately 30 minutes. Scale bar $200 \mu \mathrm{m}$.

If high flow speeds are necessary, we can resort to a dual height microfluidic device, where at least the inlets to the chamber have a lower height and consequently offer more resistance to fluid flow. In the case of a dual height microfluidic device we would naturally need to perform Comsol simulations on a three-dimensional model, which was not necessary to characterize the devices employed in our experiments.

For our simulations we focus mostly on chambers with a diameter of $200 \mu \mathrm{m}$, because preliminary experiments gave the best results in them. At first, we perform simulations of diffusion of $\mathrm{KCl}$ into the chambers with different dimensions of the inlet channels. Results of a simulation where the inlets are $10 \mu \mathrm{m}$ wide and $10 \mu \mathrm{m}$ long channels can be seen in figure 5.2. The flow of $\mathrm{KCl}$ solution into the device is started at time $t=0 \mathrm{~s}$ and is observed for 5 minutes. In this simulation we assume that the $\mathrm{KCl}$ solution is flowing into the device inlet directly at the start of the simulation - disregarding possible lag times of the pump. We find that after about $90 \mathrm{~s}$ the concentrations in the channel and the smaller chambers $(d \leq 100 \mu \mathrm{m})$ have equilibrated. The largest chambers have a diameter of $200 \mu \mathrm{m}$ and need $240 \mathrm{~s}$ for the equilibration process. 
The second series of simulations is concerned with the timescales for the diffusion of tetrameric vimentin. We perform simulations where we used both isotropic and anisotropic diffusion coefficients to determine the equilibration times. The equilibration times are very similar, as shown in figure 5.3. Therefore, we limit the following description to simulations with an anisotropic diffusion model. Figure 5.4 shows a time series of the diffusion process of vimentin tetramers. Clearly the diffusion process of tetrameric vimentin is much slower than that of the $\mathrm{KCl}$ ions. According to our simulations, the equilibration of a chamber with a diameter of $200 \mu \mathrm{m}$ lasts about 120 minutes.

In summary, the simulations give typical timescales for the diffusion processes in the microfluidic device. For $\mathrm{KCl}$ we can assume that an equilibration of the concentrations in the main channel and all chambers is completed after 5 minutes at the latest. For the diffusion of tetrameric vimentin we obtain a maximum equilibration time of about 120 minutes. Because we ultimately want to observe the formation of filaments in the chambers, a large difference between the two timescales is to our advantage. The formation of filaments is started by an increase in the $\mathrm{KCl}$ concentration, which can enter the chambers much faster than the tetrameric vimentin is able to leave them by diffusion. Now, the growing size of the vimentin assembly products leads to a decrease of the diffusion coefficient, which in turn leads to even longer diffusion times for vimentin aggregates. In the end, the largest filaments are unable to leave the chamber simply because they wont fit through the inlet channel. 


\subsection{From tetramer to filament and back in a dif- fusion chamber}

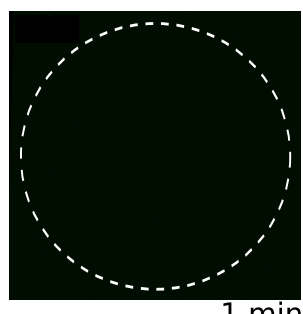

$1 \mathrm{~min}$

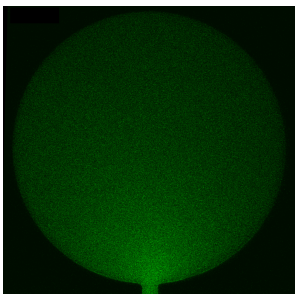

$5 \min$

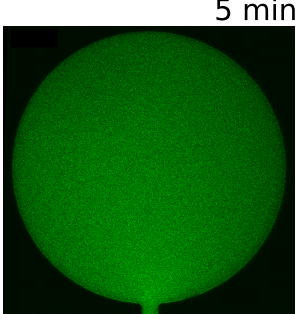

$9 \min$

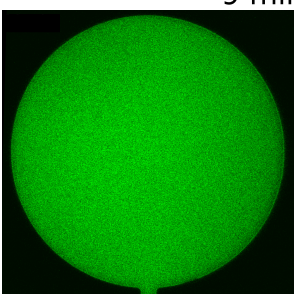

$30 \min$

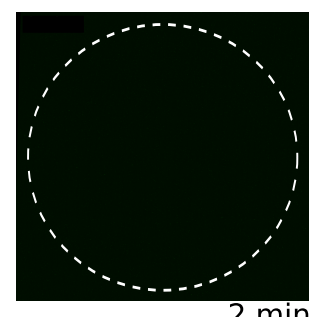

$2 \mathrm{~min}$

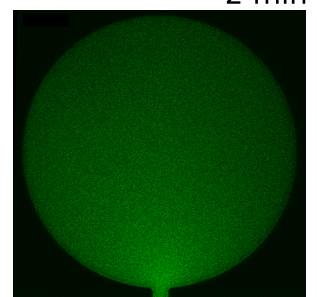

$6 \mathrm{~min}$

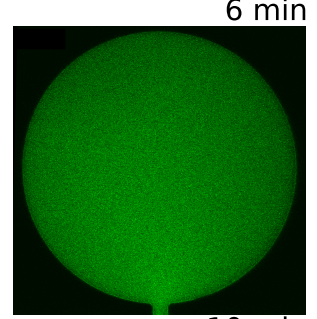

$10 \mathrm{~min}$

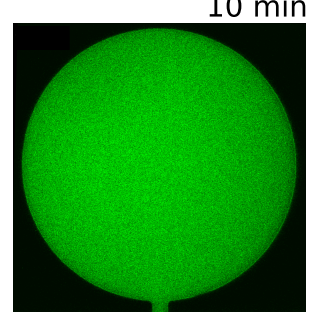

$40 \mathrm{~min}$

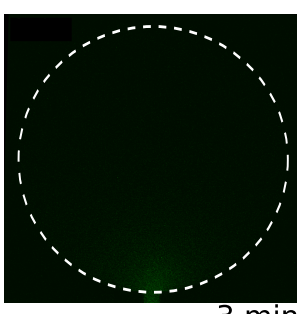

$3 \mathrm{~min}$

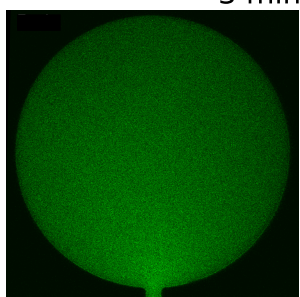

$7 \mathrm{~min}$

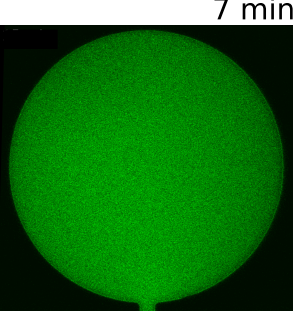

$15 \mathrm{~min}$

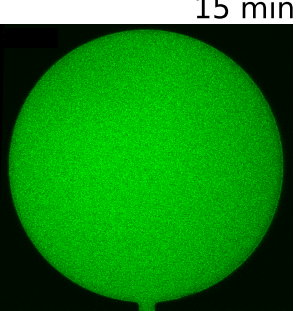

$50 \min$
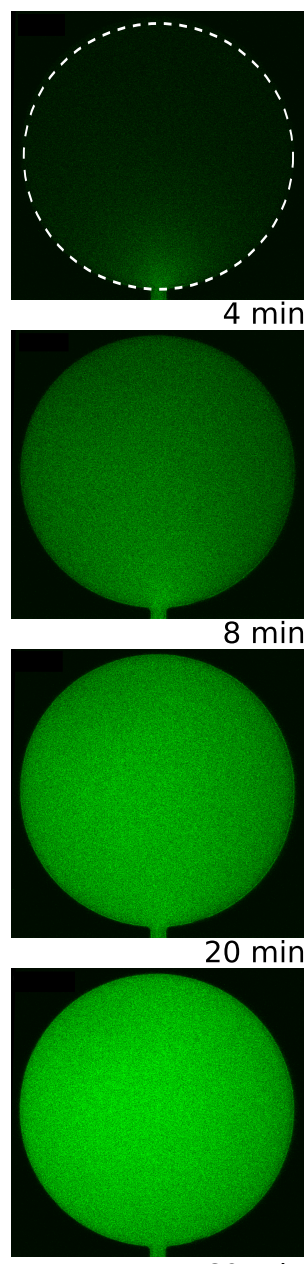

$60 \min$

Figure 5.5: Diffusion of fluorescently labeled vimentin into a chamber. Vimentin diffuses into a chamber with a diameter of $200 \mu \mathrm{m}$ (dashed line in the first four images) coated with a lipid bilayer. The equilibration process is completed after 60 minutes. At first, when a strong concentration gradient is still present, diffusion increases the concentration in the chambers rapidly. In the later equilibration process we observe only a small increase in concentration.

Here we describe the assembly and disassembly of vimentin filaments in a diffusion-chamber-device. All microfluidic experiments that are shown in this chapter are performed in microfluidic devices coated with a lipid bilayer as described in chapter 3.4. Observations are made in chambers with a diameter of $200 \mu \mathrm{m}$. As before, we refer to vimentin labeled with AlexaFlour 488 as green and to vimentin labeled with ATTO $647 \mathrm{~N}$ as red. 
The experimental phases we use are listed in detail in chapter 3.3.2, For ease of use, we reproduce an abbreviated list with identical numbering:

Step 1: coating of the device with a lipid bilayer.

Step 2: residual vesicles are removed.

Step 3: diffusion of green tetrameric vimentin into the chambers.

Step 4: the main channel is freed from residual vimentin.

Step 5: assembly of vimentin.

Step 6: salt free buffer decreases the $\mathrm{KCl}$ concentration in the chambers.

Step 7: diffusion of red vimentin into the chambers.

Step 8: the main channel is freed from residual vimentin.

Step 9: the assembly of both vimentin species is resumed/started.

Step 10: the disassembly of filaments is initiated.

\subsubsection{Diffusion of vimentin into the chambers}

The first phase of any experiment after the coating of the device is the inflow of vimentin in tetrameric form (step 3). The image series in figure 5.5 shows the inflow of green vimentin into a chamber with a diameter of $200 \mu \mathrm{m}$. To characterize the inflow timescales, we take the average intensity over the whole chamber and plot the normalized value over time in figure 5.6. For the purpose of comparison, the figure also contains normalized data from our simulations. When we compare both curves, we find that the diffusion process proceeds much faster for the experimental data set. Consequently, it is likely that we have estimated the diffusion coefficient for the simulations to low. Alternately, an increased temperature and consequently a reduced viscosity of the aqueous solution might be responsible for the faster equilibration process.

The quicker equilibration of vimentin between main channel and chamber is, of course, advantageous for the initial filling of the chamber. However, a slower diffusion process would decrease the loss of protein during e.g. the $\mathrm{KCl}$ diffusion phase. 


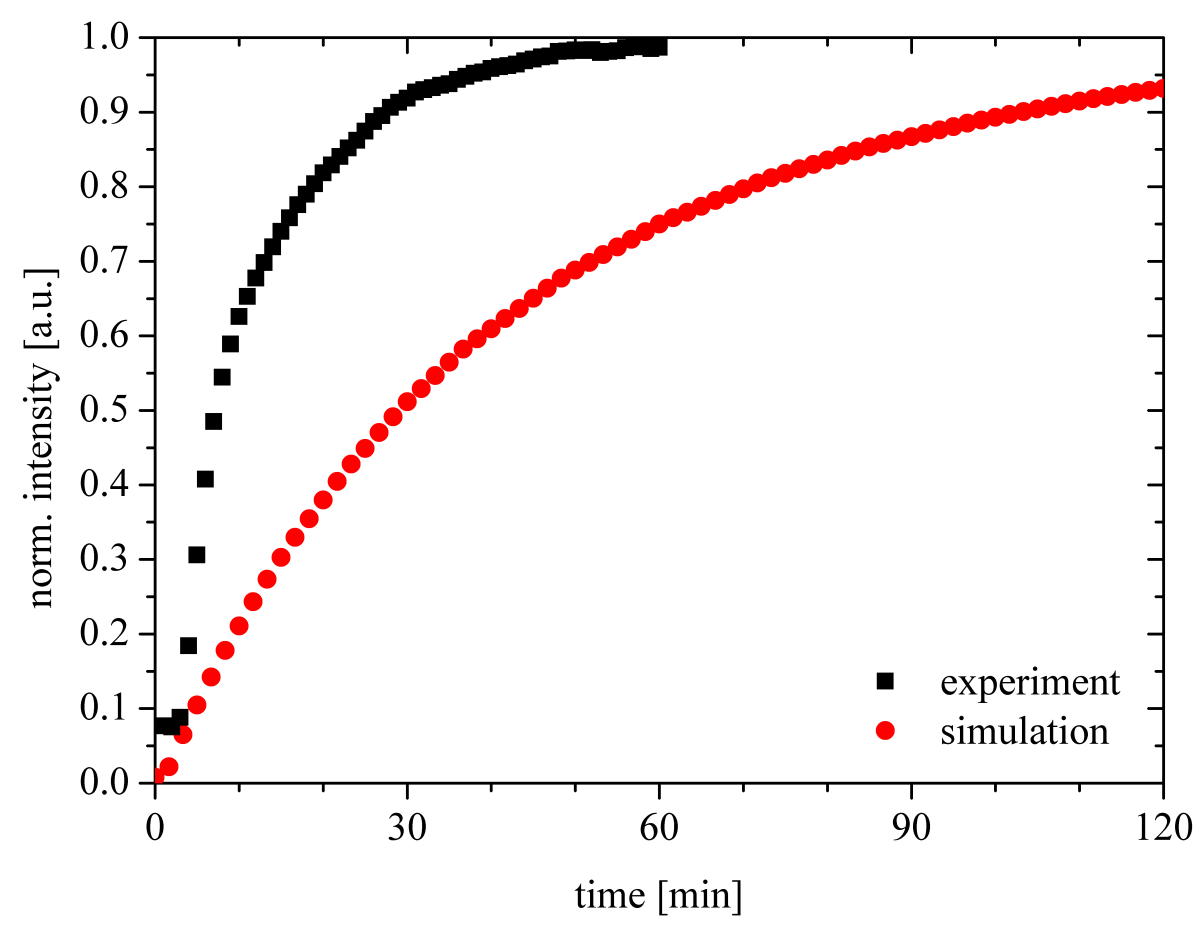

Figure 5.6: Diffusion timescales in experiment and simulation. The average fluorescence intensity and vimentin concentration in a chamber with diameter of $200 \mu \mathrm{m}$ measured in experiment and simulation. Data are normalized with the maximum value. The intensity curve for the experimental data is much steeper than the curve for the simulation and leads to an earlier equilibration in the experiment, indicating a faster diffusion process for the experimental setup.

\subsubsection{Assembly of vimentin}

After the tetrameric vimentin has entered the chamber by diffusion and residual vimentin has been flushed from the main channel, the assembly process is initiated by flushing $100 \mathrm{mM} \mathrm{KCl}$ solution through the main channel in steps 4, 5. As shown in figure 5.2, we expect the $\mathrm{KCl}$ concentration to equilibrate within 2 minutes. Assembly of vimentin begins to set in at $\mathrm{KCl}$ concentrations below $100 \mathrm{mM}$ [87. However, the time until the final concentration is reached is very short, so this effect is minimal. A time series of the assembly process is shown in figure 5.7 . The initial decrease of fluorescence intensity we observe can be attributed to loss of tetrameric vimentin or unit length filaments which have a similar diffusion coefficient $\left(D_{t}=2.4 \times 10^{-11} \mathrm{~m}^{2} / \mathrm{s}\right.$ and $D_{U L F}=1.3 \times 10^{-11} \mathrm{~m}^{2} / \mathrm{s}$, respectively $)$. We also observe that the fluorescence intensity over the whole chamber becomes less homogeneous 


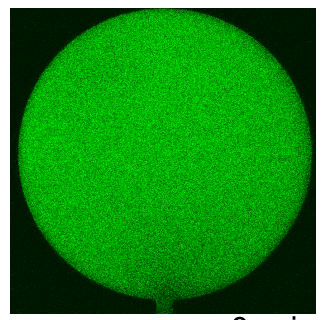

$0 \mathrm{~min}$

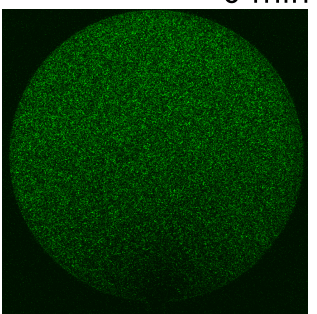

$60 \mathrm{~min}$

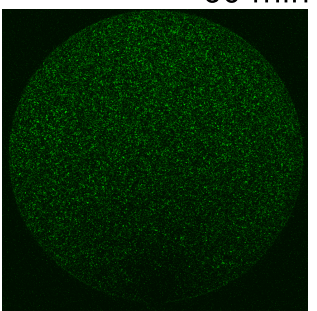

$120 \mathrm{~min}$

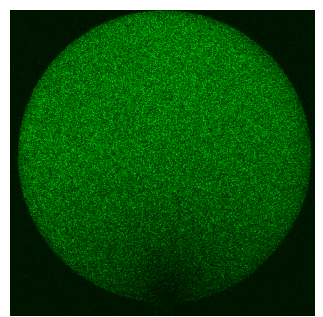

$15 \mathrm{~min}$

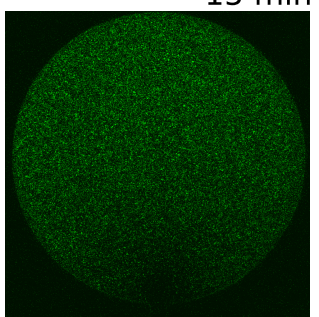

$75 \mathrm{~min}$

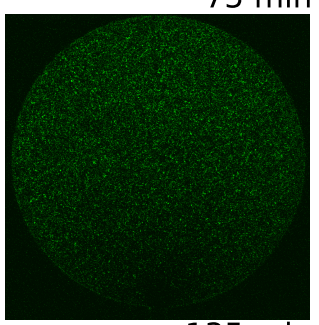

$135 \min$

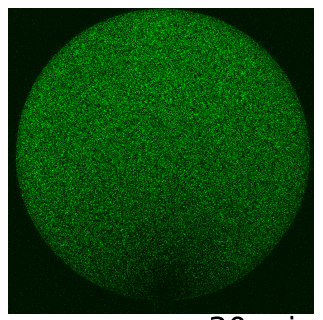

$30 \mathrm{~min}$

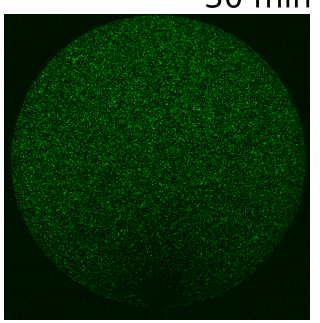

$90 \mathrm{~min}$



$150 \mathrm{~min}$

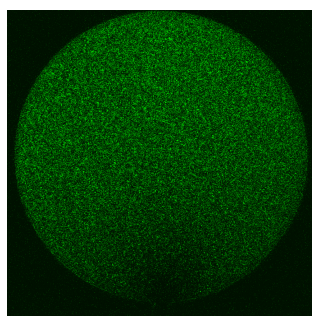

$45 \mathrm{~min}$
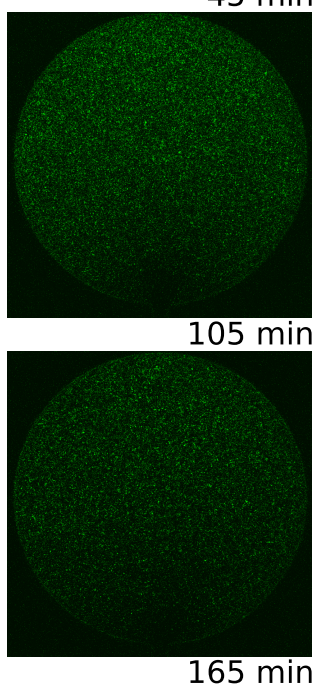

Figure 5.7: Assembly of vimentin in a chamber observed over time. $\mathrm{KCl}$ flow begins at time 0 . Shortly thereafter $\mathrm{KCl}$ begins to diffuse into a chamber with a diameter of $200 \mu \mathrm{m}$ and thus initiates the filament assembly process. The time series shows the progress of the assembly reaction. In the first minutes, a certain amount of protein is lost due to diffusion, as can be seen from the decreasing fluorescence intensity. After about 30 minutes the vimentin aggregates have become so large that protein loss becomes minimal. Also, we observe that the homogeneity of the fluorescence intensity decreases over time. We use this effect to characterize the progress of the assembly reaction.

with progressing time. We can interpret this as the beginning of the assembly reaction and thus the formation of vimentin filaments. To quantify the assembly process and to determine typical timescales, we measure the standard deviation of the fluorescence intensity over the whole chamber and plot it over time. With this approach we can quantify the change from a tetrameric vimentin solution, which results in homogeneous fluorescence intensity over the whole chamber to a vimentin filament solution with a more heterogeneous fluorescence intensity distribution [88]. However, this method is not suitable to quantify the elongation process of, especially long, filaments. For example, two individual filaments or a single filament of equal total length give the same standard deviation value.

The course of the standard deviation is plotted in figure 5.8. From this plot, we can also calculate typical timescales of the assembly process. To this end, we 


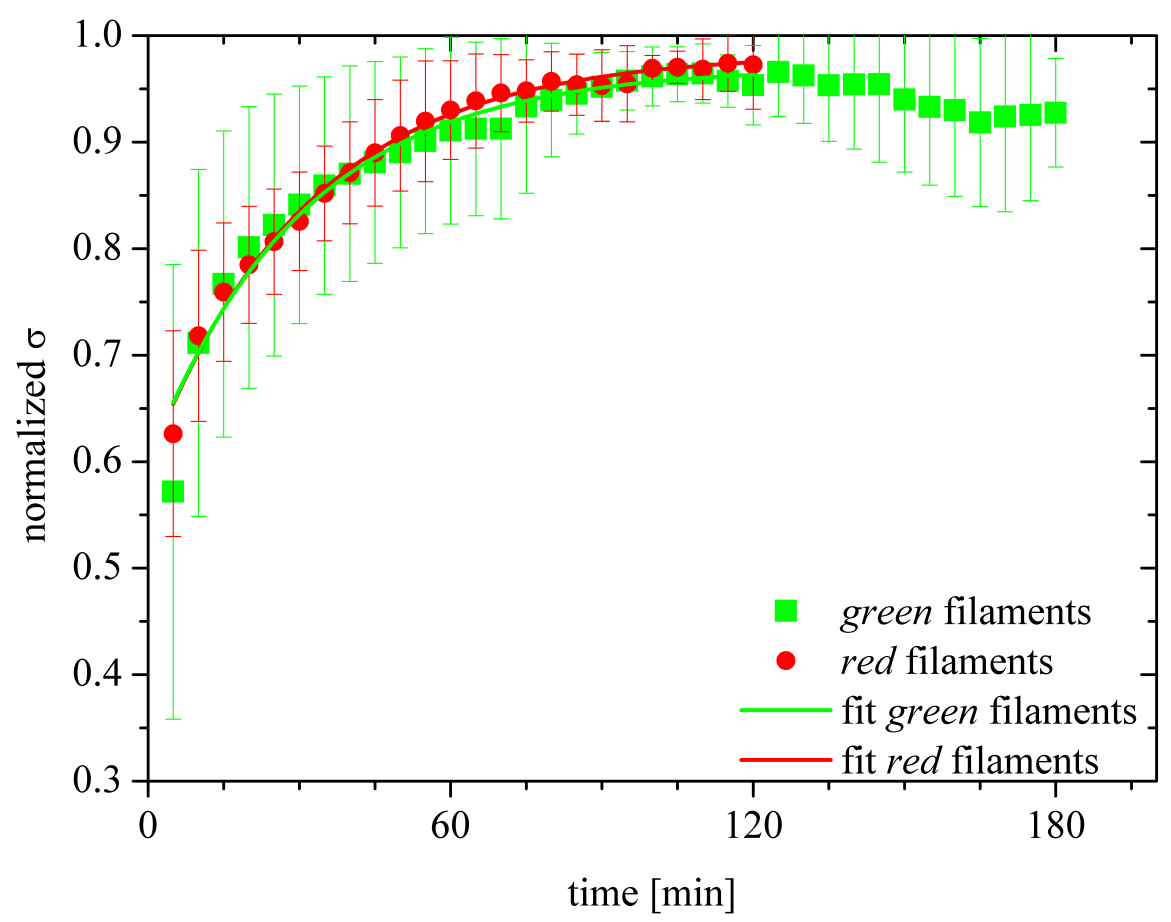

Figure 5.8: Quantification of the assembly process in chambers. The standard deviation of the fluorescence intensity in a chamber is used as a measure for the assembly state. Average values for two different assembly steps are shown. The first assembly step begins with only tetrameric vimentin. The second assembly step begins after fresh, red tetramers are added to the already assembled, green filaments of the first assembly step. We can observe the same assembly behavior for both cases. An exponential fit yields time constants $\tau_{1 / 2}=20 \mathrm{~min}$ for the assembly process.

fit the graphs with the exponential function $f(x)=a \cdot \exp (-t / \tau)+y$, where $\tau$ is the characteristic time of the assembly process. From this characteristic time we calculate the half-value time of the assembly process $\tau_{1 / 2}$. At this point, we can compare the assembly characteristics of the two different assembly processes we observe in the chamber with regard to their timescales. The first assembly process is started, when only green tetrameric vimentin is present in the chamber (step 5). A second assembly process is initiated after fresh, red tetrameric vimentin is added to the already assembled filaments (step 9). As shown in figure 5.8, the two curves, as well as the timescales, $\tau_{1 / 2}=20$ minutes, agree very well.

In figure 5.9 the progress of the assembly reaction is shown from the beginning of the second assembly step. The green filaments, which are already present, show little to no change in the assembly state when taking into account the large measure- 


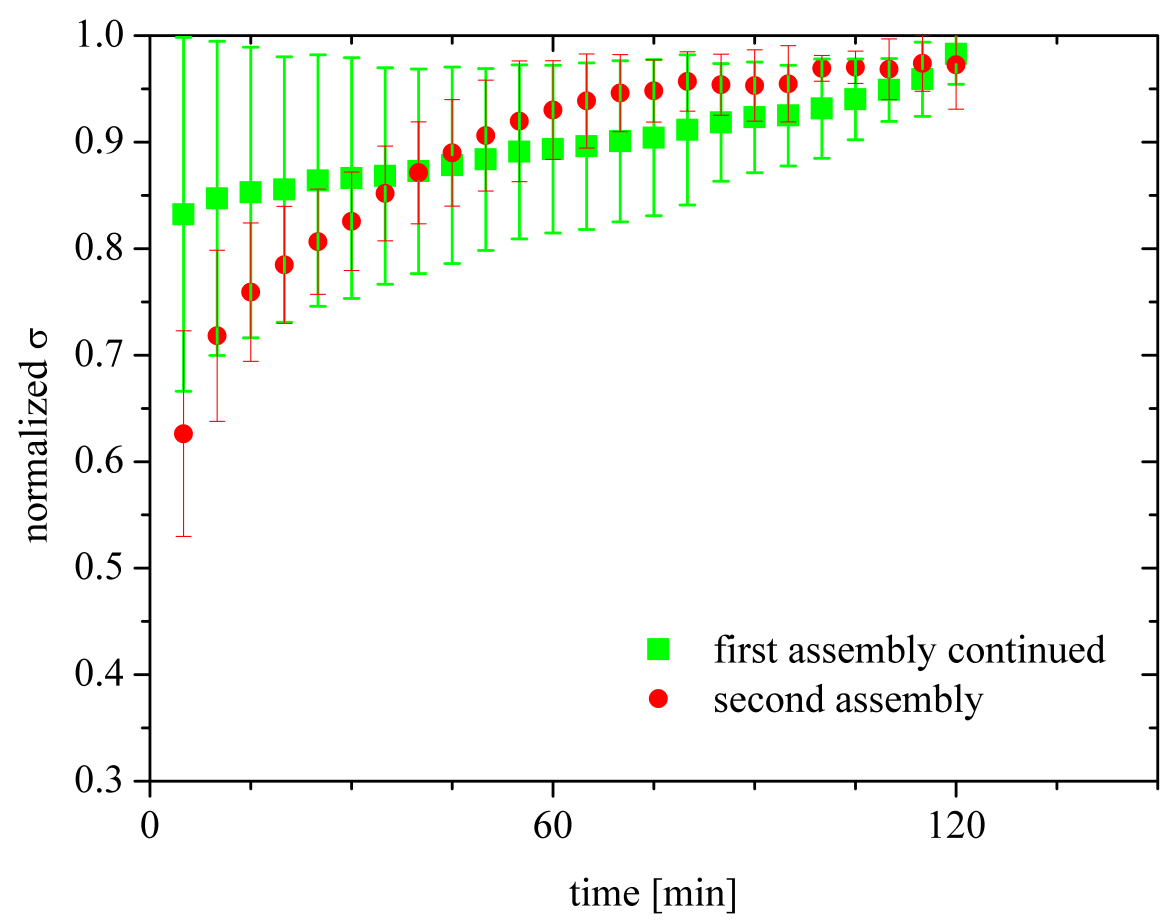

Figure 5.9: Continued assembly after addition of fresh tetramers. The green curve shows the average of the progressing assembly of the first batch of tetrameric, green vimentin. The red curve shows the average of the freshly started assembly reaction of the second batch of tetrameric, red vimentin. When accounting for the rather large error bars of the green curve, the assembly reaction does not show much, if any, change. The second assembly phase progresses similar to the first, as already shown in figure 5.8 .

ment error. This is expected, given that the method used to quantify the assembly mainly tracks the early phase of the assembly, where free tetrameric vimentin is incorporated into filaments with lengths of a few micrometers. To assess the quality and length of the filaments in the chambers, we show several images of typical assembly results in figure 5.10. Images 5.10 a) and b) show filaments in the chambers after 2 respectively 3 hours of assembly. Individual filaments are difficult to discern in the images acquired in diffusion chambers. When compared to pre-assembled filaments flushed into the chamber as shown in figure $5.10 \mathrm{~d}$ ), the morphology of the filaments assembled in the chambers is clearly different from those assembled in dialysis tubing or a reaction tube. Figure 5.10 e) and f) show filaments adsorbed to a cover slip after an assembly time of 2 hours at $20^{\circ} \mathrm{C}$ and $37^{\circ} \mathrm{C}$ in a reaction tube. As becomes clear from these images, the quality of the filaments and images is inferior in the case of the diffusion-chambers. However, the filaments assembled at 
a)

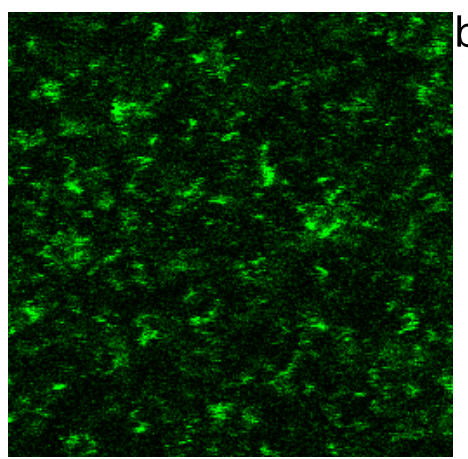

d)

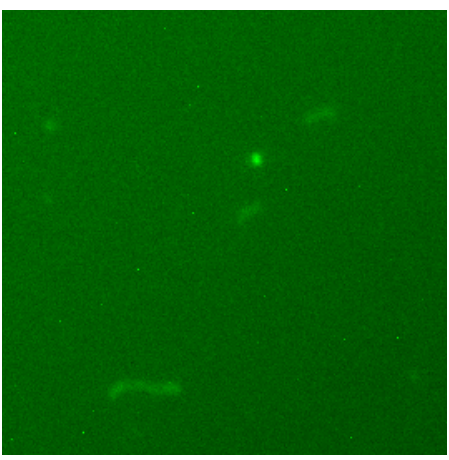



e)

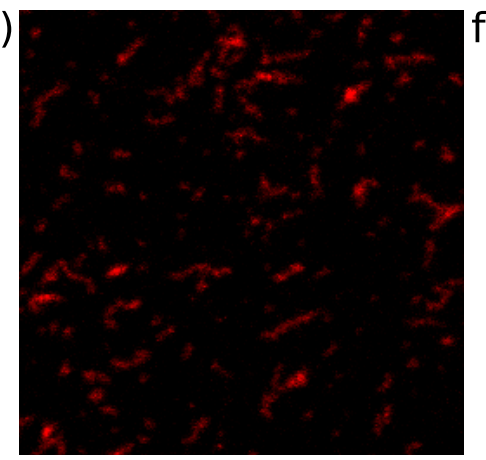

c)
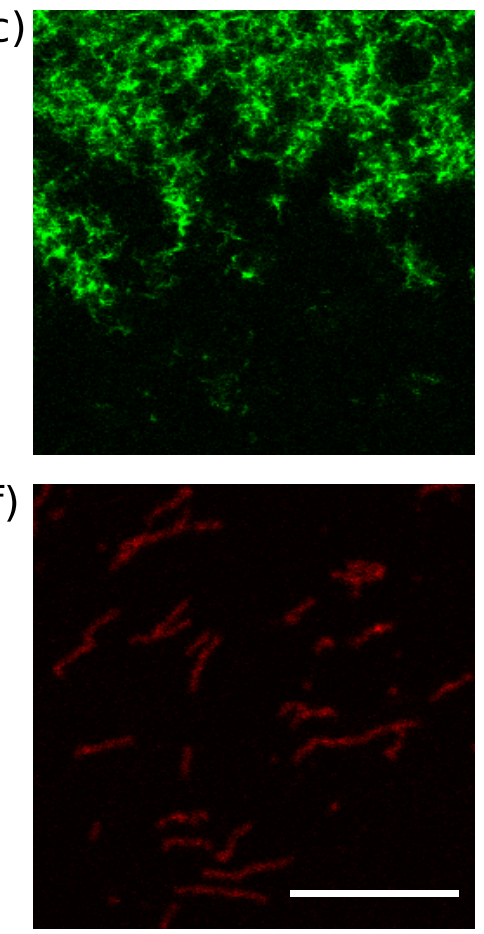

Figure 5.10: Images of vimentin filaments assembled under different conditions. a) Green vimentin filaments after 2 hours of assembly in a diffusion chamber. b) Assembly state of a) after addition of red tetramers and further 3 hours of assembly. c) Green filaments assembled for 20 hours in a diffusion chamber. d) Pre-assembled filaments flushed into a chamber and imaged using epi-fluorescence. e) Filaments assembled in a reaction tube at $20^{\circ} \mathrm{C}$ for 2 hours. f) Filaments assembled in a reaction tube at $37^{\circ} \mathrm{C}$ for 2 hours. Scale bar shows $10 \mu \mathrm{m}$

$20^{\circ} \mathrm{C}$ in a reaction tube are similar in appearance to those assembled in a chamber after 2 hours. However, extended assembly times in a diffusion chamber do not lead to "normal" filaments, but instead larger aggregates form, as shown in figure $5.10 \mathrm{f}$ ).

\subsubsection{Disassembly of vimentin}

While the assembly process of vimentin has been characterized rather comprehensively [9 15], the disassembly process is not as well understood. One of the questions that we set out to answer with the diffusion-chamber-microfluidic-devices is whether the filaments disassemble only from the endpoints or whether they can break in the middle. Or, formulated differently, if the assembly and disassembly process are inverse processes. Here, elongation by addition of individual unit length filaments corresponds to disassembly by removal of individual unit length filaments. End-toend annealing corresponds to a break in the filament, resulting in two individual, 


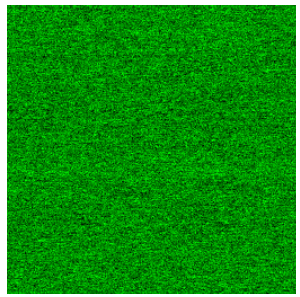

$0 \mathrm{mM}$

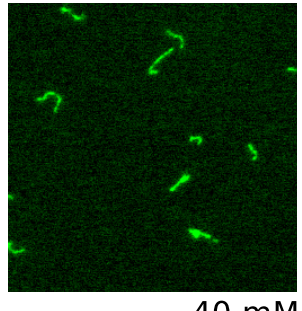

$40 \mathrm{mM}$

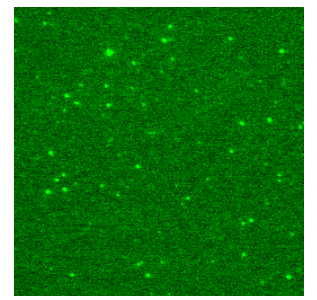

$10 \mathrm{mM}$

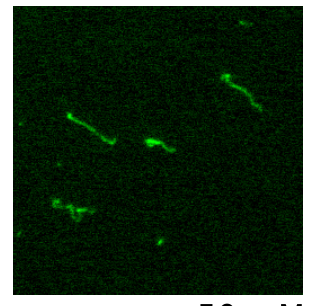

$50 \mathrm{mM}$

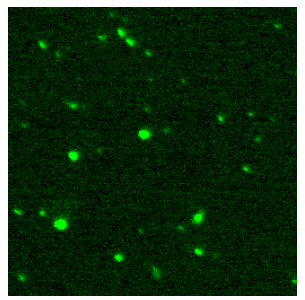

$20 \mathrm{mM}$

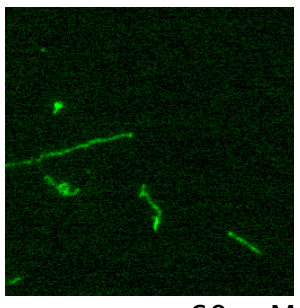

$60 \mathrm{mM}$

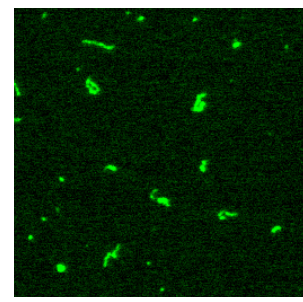

$30 \mathrm{mM}$

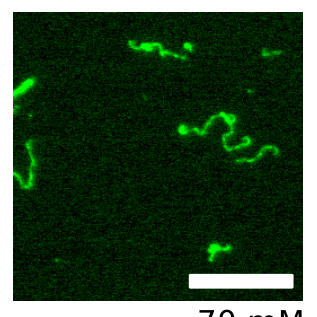

$70 \mathrm{mM}$

Figure 5.11: Vimentin filaments after 20 minutes at different $\mathrm{KCl}$ concentrations. $\mathrm{KCl}$ concentrations are reduced by dilution into salt free buffer. At $\mathrm{KCl}$ concentrations of $30 \mathrm{mM}$ or less, we observe a clear reduction of filament length. For concentrations of $40 \mathrm{mM}$ or more, a disassembly process is not apparent after short times. Notably, salt concentration reduction by dilution also reduces the vimentin concentration. Scale bar $10 \mu \mathrm{m}$.

shorter filaments.

\section{Preliminary bulk experiments}

The disassembly process of the filaments is initiated by reducing the $\mathrm{KCl}$ concentration in the filament solution through dilution in a reaction tube or through dialysis into $\mathrm{KCl}$ free buffer. Figure 5.11 shows the progress of the disassembly reaction 20 minutes after the dilution for different $\mathrm{KCl}$ concentrations. The $\mathrm{KCl}$ concentration is reduced by diluting the sample with $\mathrm{KCl}$ free buffer. It is therefore inevitable that the protein concentration is also strongly reduced, if we want to dilute so far that we obtain a $\mathrm{KCl}$ concentration of less than $1 \mathrm{mM}$. To obtain comparable data, all samples are diluted to the same, final vimentin concentration.

For small reductions of the $\mathrm{KCl}$ concentration, as shown in figure 5.11, we do not observe the onset of the disassembly process. However, for concentrations below $30 \mathrm{mM}$, we can clearly see that the average filament length reduces or that the filaments disassemble altogether. The comparison of the different salt concentrations indicates that the disassembly speed increases with lower $\mathrm{KCl}$ concentration. By imaging the same sample after about 120 hours, this assumption is strengthened. We observe a further reduction of the average filament length for $\mathrm{KCl}$ concentrations 
below $50 \mathrm{mM}$ (data not shown).

The disassembly process described above is performed in bulk solutions in a reaction tube. However, vimentin filaments can be adsorbed to, for example, a glass cover slip and then flushed with $\mathrm{KCl}$ free buffer. In this case, the disassembly process does not set in as it does in the bulk sample. In fact, we have not observed a disassembly processes on filaments already adhered to a glass cover slip induced by reduction of salt concentration. This points to a strong interaction of the filaments with surfaces, which can influence their behavior significantly. Consequently, it is very important to prevent the filaments from adhering to surfaces when we want to observe the disassembly process or make other observations on the assembly or disassembly reaction of the filaments.

As an alternative to the reduction of the $\mathrm{KCl}$ concentration in a reaction tube by dilution or flushing a cover slip with salt free buffer, it is also possible to place the protein solution in dialysis tubing and dialyze it into buffer of the appropriate $\mathrm{KCl}$ concentration. We avoid the dilution of the protein and at the same time maintain a small surface-area-to-volume ratio to avoid surface effects. However, this approach has two pitfalls. First, the equilibration process in the dialysis tubing typically lasts 2 hours. Secondly, during the equilibration process the $\mathrm{KCl}$ concentration is heterogeneous throughout the sample. Because the disassembly reaction in a reaction tube proceeds faster than the dialysis process and the possible $\mathrm{KCl}$ concentration dependence of the process, macroscopic dialysis is not suitable for a detailed analysis of the disassembly process. Nonetheless, we can observe that filaments at standard concentrations disassemble completely when they are dialyzed into $\mathrm{KCl}$ free buffer within no more than 60 minutes. Also, the real time observation of the filament disassembly process in dialysis tubing poses further problems that are easy to avoid through the use of diffusion-chambers.

\section{Disassembly in chambers}

The diffusion-chamber-microfluidic-device can be used for the observation of the disassembly process of vimentin filaments. The exchange of salt ions in the chambers occurs on short timescales without producing long-lasting or strong concentration gradients. Also, the filament concentration in the chambers remains constant. Only with the onset of a disassembly reaction the freed subunits start to leave the chamber by diffusion. However, because we expect the disassembly process to happen on short timescales, this poses no major problem. 




$5 \mathrm{~min}$

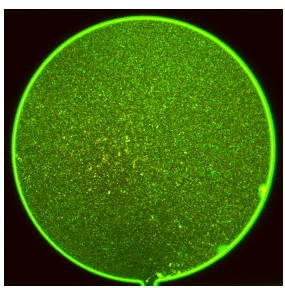

$30 \mathrm{~min}$

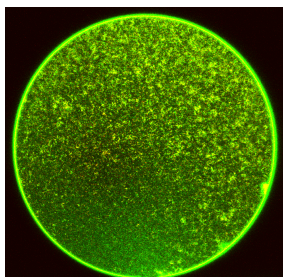

$10 \mathrm{~min}$

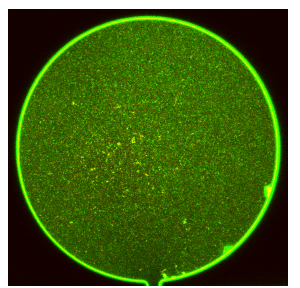

$60 \mathrm{~min}$

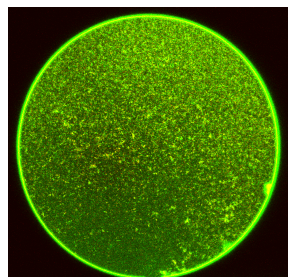

$15 \mathrm{~min}$

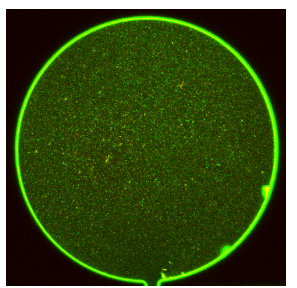

$120 \mathrm{~min}$

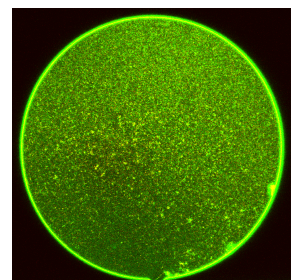

$20 \mathrm{~min}$

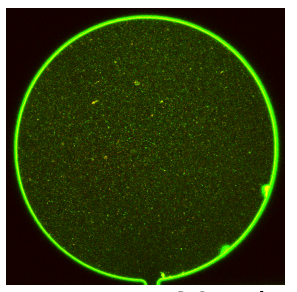

$180 \mathrm{~min}$

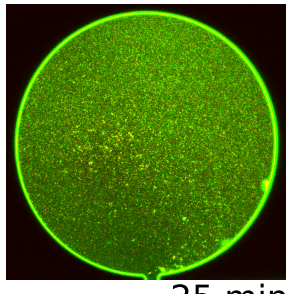

$25 \mathrm{~min}$

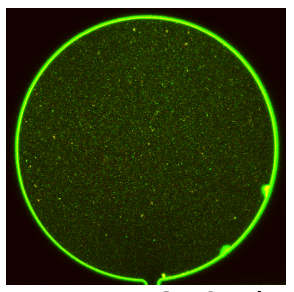

240min

Figure 5.12: Disassembly process in a diffusion chamber with $200 \mu \mathrm{m}$ diameter over time. Flow of salt free buffer sets in at time 0 . The disassembly process sets in after 5 to 10 minutes. After about 30 minutes most of the visible, large aggregates have disassembled. Remains of the filaments are found in the chamber even more than 240 minutes after the start of the disassembly process.

Ideally, the disassembly process in diffusion chambers would be observed on easy to image, pre-assembled filaments, which are flushed into the chambers. However, the lipid bilayer coating instantly ruptures when exposed to air and consequently we can not flush filament solution into empty chambers and at the same time retain the lipid bilayer coating. Therefore, we use filaments already assembled in diffusion-chambers as described in chapter 5.2.2. The disassembly process is initiated by flushing with $\mathrm{KCl}$ free buffer through the main channel (step 10). From our preliminary experiments we expect a fast disassembly process of the filaments. Filament breakdown products should leave the chamber within 60 minutes when we consider the diffusion into the chamber and out of it to be inverse processes.

We observe the disassembly process over long timescales. Images of a chamber with disassembling filaments are shown in figure 5.12. Contrary to our expectations from preliminary experiments, changes over short timescales were not always observed. In some cases, such as shown in figure 5.12, the disassembly process happens within 2 hours. In other cases, disassembly in one chamber sets in normally and at the same time the disassembly process fails to begin in a neighboring chamber in the same device despite identical experimental conditions apart from observation with the fluorescence microscope.

To quantify the disassembly process, we use the fluorescence intensity in the diffusion chambers as indicator for the remaining protein. As the smaller subunits 


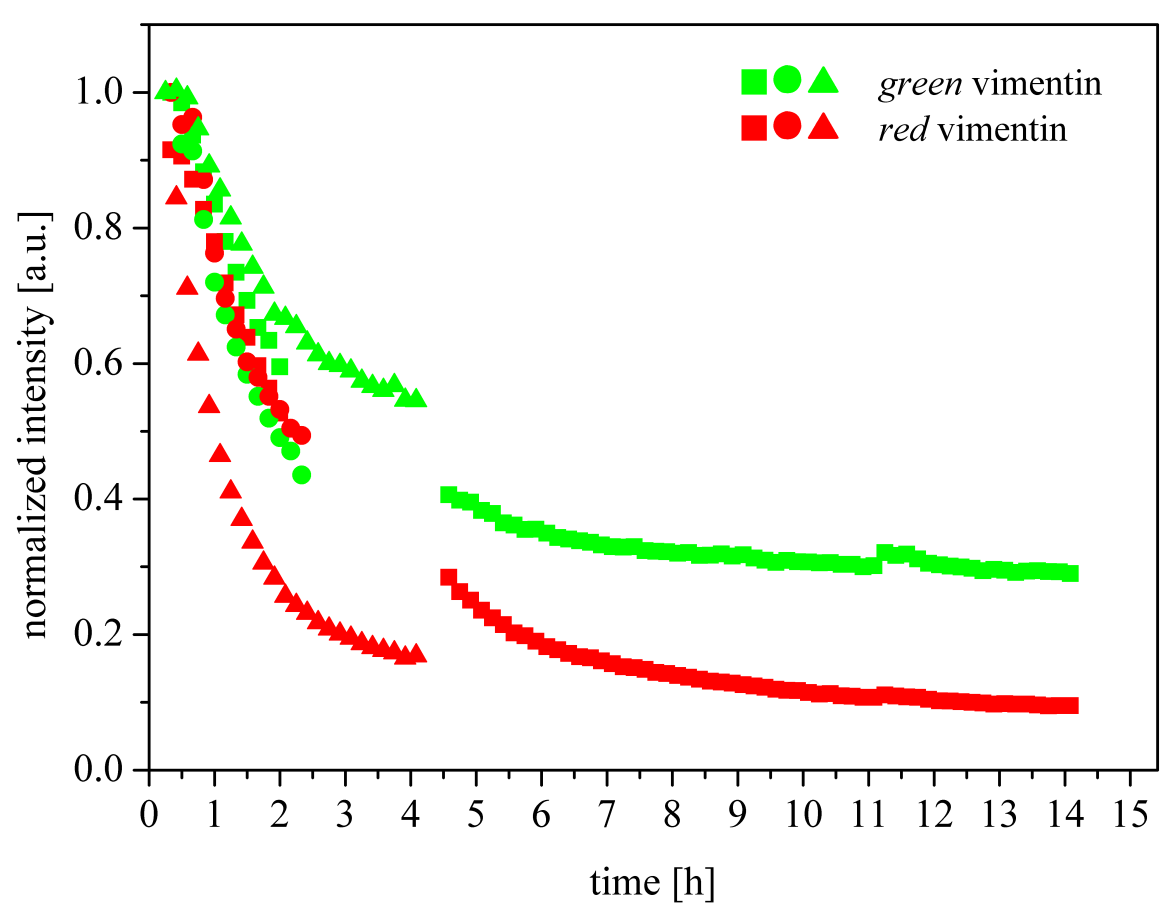

Figure 5.13: Fluorescence intensity during the disassembly process decreases over time. The average fluorescence intensity in the diffusion chamber can be used to quantify the disassembly process. Disassembly proceeds differently for the three representative cases shown here. Red and green filaments disassemble at different speeds. Red filaments can disassemble faster or slower than green filaments. Disassembly times are in the range of hours in all cases.

which are freed in the disassembly process leave the chambers within a comparably short time, this method yields a good measure of the progress of the disassembly process. To exclude that bleaching of the fluorescent dye has an effect, we perform measurements with different time intervals between individual images and therefore different bleaching effects. We find, that bleaching has no notable effect on our measurement.

\subsection{Discussion}

\subsubsection{Inflow of vimentin and characteristic timescales}

The comparison of the diffusion speeds of vimentin in a simulation and in the real device shows differences: equilibration times in the device are only half as long 
as predicted by our simulations. Consequently, it is likely that we have estimated the diffusion coefficient for the simulations to low by a factor of $\approx 2$. Alternately, an increased temperature and, in consequence, a reduced viscosity of the aqueous solution might be responsible for the faster equilibration process.

The faster equilibration of vimentin between main channel and chamber is, of course, advantageous for the initial filling of the chamber. A slower diffusion process would decrease the loss of protein during e.g. the $\mathrm{KCl}$ diffusion phase. However, the ratio of the diffusion coefficients of $\mathrm{KCl}$ and vimentin is fixed. Therefore, an adaption of the device influences only the absolute times the equilibration between channel and chamber need to complete. In summary, the diffusion-chamber-device we use shows diffusion timescales that are compatible with our goals.

\subsubsection{Assembly of vimentin and subunit exchange}

We can observe and qualitatively describe the assembly process in the diffusionchamber-device. We find that the presence of already assembled green vimentin has no impact on the early assembly process of freshly added red vimentin. The assembly process begins quickly after the flow of $\mathrm{KCl}$ through the channel is initiated. This agrees well with our simulations, which predict a lag time below 5 minutes. Also, bulk experiments show that the initial assembly steps occur within seconds 10, 15. The assembly products we obtain after 2 hours look similar to those obtained in a reaction tube kept at $20^{\circ} \mathrm{C}$ for the same time. However, even long assembly times lead only to aggregates of vimentin instead of well defined filaments. Also, the filaments are free to move in solution and not adsorbed on a surface and therefore more difficult to image than adsorbed filaments. As a result, the very short filaments that we obtain are not suitable for the detailed observation of the subunit exchange process.

Bulk and diffusion chamber experiments differ in their respective surface-areato-volume ratio. The diffusion-chambers we used in our experiment are characterized by large surface-area-to-volume ratios of typically

$$
\frac{A_{c h}}{V_{c h}} \approx 50 \cdot \frac{1}{\mathrm{~mm}} \text {. }
$$

In contrast, in a reaction tube for $100 \mu \mathrm{L}$ solution we find a surface-area-to-volume ratio of

$$
\frac{A_{r t}}{V_{r t}} \approx 1 \cdot \frac{1}{\mathrm{~mm}}
$$


The larger surface-area-to-volume ratio in the diffusion chambers is likely responsible for the less than ideal assembly process. We can safely assume that surface interactions are very relevant, because the surface treatment of the chambers has a large impact on the assembly process. When we do not use the lipid bilayer treatment, we do not obtain any filaments after an "assembly" step. Thus, while the lipid bilayer coating provides the best properties for assembly of vimentin filaments in a diffusion-chamber-device so far, conditions are still not ideal for the assembly of long, easily observable vimentin filaments.

As already described in chapter 5.2.3, it would be ideal to use pre-assembled green filaments and introduce red tetrameric vimentin by diffusion to observe a subunit exchange process. However, the same problems as before apply in this case.

\subsubsection{Disassembly of vimentin}

To evaluate if the timescales of the disassembly process of about 2 hours we measure experimentally are plausible, we have to look at the different timescales that are involved. First, $\mathrm{KCl}$ needs to leave the chambers by diffusion. This process is inverse to the diffusion of $\mathrm{KCl}$ into the chambers and therefore occurs on the same timescales. With the data from our simulations in chapter 5.1 we can be certain that after 5 minutes the $\mathrm{KCl}$ concentration is practically zero. Furthermore, in our preliminary experiments we have both observed the disassembly process on strongly diluted filaments as well as filaments at normal concentration in dialysis tubing. In both cases, we can make the conservative estimate that the disassembly process is finished at the latest after 60 minutes. Finally, to detect the decreasing fluorescence intensity the protein disassembly product has to leave the chambers by diffusion. As shown in chapter 5.2.1, the inflow of tetrameric vimentin takes about 60 minutes to reach an equilibrium state. Our simulation indicates a somewhat slower equilibration. So, to estimate an upper limit for the time it takes to complete process from equilibration of to remove $\mathrm{KCl}$, the actual disassembly process and the removal of the disassembly products we add all estimated timescales. The resulting estimate for the slowest possible complete disassembly process is a duration of about 2 hours. If we compare our estimated time scale to that found in our measurements, as shown in figure 5.13, we observe a much slower decrease of fluorescence intensity during the disassembly process. Because of the rather pronounced differences between the three experimental scenarios, diffusion chamber, dialysis tubing and dilution in a reaction tube we list below, it is difficult to determine the "right" experimental 
disassembly process. This also means that it is difficult to find the "right" timescales of the disassembly process. Studies describing the disassembly process of vimentin filaments are largely missing and we are thus unable to offer a comparison with literature data. We instead consider the different (preliminary) experiments we have conducted. The $\mathrm{KCl}$ concentration reduction, both in diffusion chamber and reaction tube occur within minutes. The dialysis tubing in contrast takes long to equilibrate. The protein concentration is changed in the reaction tube and remains more or less constant in both other cases. Finally, the ratio of surface-area to volume is largest in the diffusion chambers. Despite the lipid bilayer coating, the interaction between vimentin filaments and chamber surfaces might still be so strong that the results of the disassembly experiment were heavily influenced. In comparison the surfacearea-to-volume ratio is very small in both dialysis tubing and reaction tube. In the end, it is difficult to decide which of these parameters influences the disassembly process in unexpected ways and lead to different experimental results.

\subsubsection{The diffusion chamber device as a whole}

The idea of the diffusion-chamber-device is very appealing and manifold applications in a biophysical context are conceivable. Here, we have discussed different applications for vimentin intermediate filaments and obtained promising first results. However, while the flexibility of the microfluidic device is certainly a strong point, experimental parameters still need to be refined. The inherently large surface-areato-volume ratio of the diffusion-chamber-device is problematic. Thus, we needed to find a surface coating method that allows for long-time observation of vimentin filaments. However, a lipid bilayer is the most suitable choice thus far. 


\section{Chapter 6}

\section{Filaments in a confining geometry}

After the assembly and disassembly in chambers and the exchange of subunits, we consider the mechanical properties of vimentin filaments. To this end, we confine vimentin filaments in microchannels of different widths and heights. The confining channel geometry offers distinct advantages: we can investigate a scaling law formulated 30 years ago experimentally and study the mechanical properties of the filaments in detail. Also, data analysis of the filament contours is straightforward.

Significant parts of our study on confined vimentin filaments have been published in Physical Review Letters [89].

\subsection{Polymer physics}

It is not surprising that physicists have developed theoretical descriptions that provide access to the characteristic properties of polymers. The choice of the model used to describe a polymer depends on the basic properties of the filament. Very stiff polymers or very flexible polymers are best described by different models. Also, the circumstances of the observation have an impact - is our primary interest the macroscopic or microscopic description of the polymer.

There are two distinct model types, which are either based on discrete subunits, in the fashion of chain links, or a continuous elasticity ansatz. The most basic assumptions for a polymer with distinct links leads to the ideal chain model, which is best described as a "random walk polymer". This model ignores a possible orientational correlation between the individual polymer segments that is often present in real polymers. 


\subsubsection{The worm-like chain}

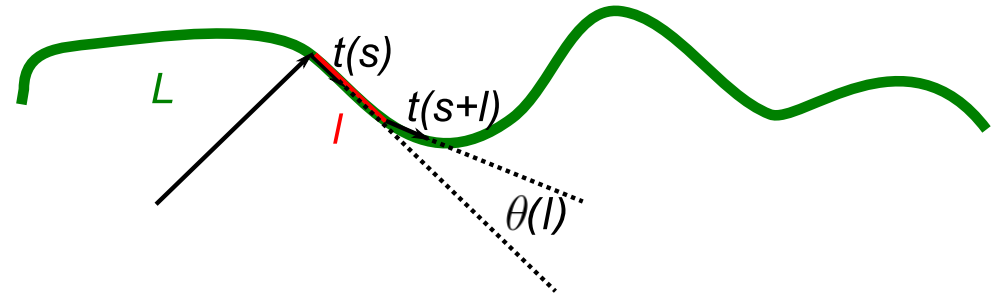

Figure 6.1: Filament with arc length parametrization. Hero, $L$ is the contour length of the filament, $t$ is the tangent vector and $l$ the distance between two tangents. $\theta$ is the angle between two tangent vectors.

Polymers described as an ideal chain are only flexible at the joints of the chain links. This restriction is in most cases contrary to the reality of the molecular properties of the polymers. Polymers such as actin, DNA or intermediate filaments can better be described as thin continuous elastic lines obeying Hookes elasticity law with regards to their bending stiffness. Here, we take into account only small deformations. Also, we limit our description to the bending properties of the filaments and disregard stretching of the polymer. This also agrees with our experimental parameters, where we do not apply an external stretching force that would require such a term.

To obtain a continuous description of a polymer with chain link length $l_{k}$ and number of chain links $N_{k}$ we take the limit of the chain link length $l_{k}$ :

$$
l_{k} \rightarrow 0
$$

To keep the polymer length $N_{k} l_{k}$ constant, we also take the limit

$$
N_{k} \rightarrow \infty
$$

The worm-like chain model, which results from these assumptions is described by the Hamiltonian

$$
\mathcal{H}_{c}[z(x)]=\int_{0}^{L_{x}} d x\left[\frac{\kappa}{2}\left(\frac{\partial^{2} z(x)}{\partial x^{2}}\right)^{2}\right] .
$$

To describe the filament contour, we can use the arc length representation as shown in figure 6.1. Here, $s$ is the distance from the origin of the polymer along the polymers contour. The worm-like chain model assumes that movements in the $x-y$ plane and the $x-z$ plane decouple.

The directional correlation of the polymer is given by $\langle\cos \theta(l)\rangle$. Here $\theta(l)$ is the angle between two tangents of the filament located a distance $l$ from each other. The 
average correlation between two given tangents decreases with increasing distance along the filament contour. For the worm-like chain model we find an exponential decay of the correlation with the decay constant $L_{P}$, the so called persistence length [90]:

$$
\langle\cos \theta(l)\rangle=\exp \left(-\frac{|l|}{2 L_{P}}\right)
$$

The persistence length can also be set into relation with the bending rigidity $\kappa$

$$
L_{P}=\frac{\kappa}{k_{B} T}
$$

Thus, the persistence length is the bending rigidity in terms of $k_{B} T$. Because, our experiments are always performed at room temperature, we do not add an index specifying the temperature to $L_{P}$.

\subsubsection{The confined worm-like chain}
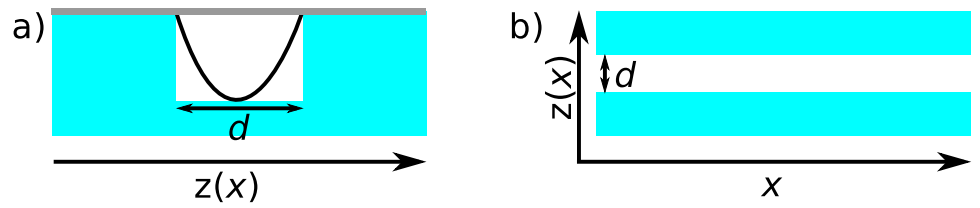

Figure 6.2: a) The wall potential we assume in a microchannel. b) The coordinate system used for filaments in a microchannel. Both images show the channel width $d$.

The model we have described in the previous section does not included the effects of a confining channel geometry. Thus, we now consider filaments confined in narrow channels. The theoretical description of confined filaments is based on references $36,91,93$. The microchannels fabricated as detailed in chapter 3.2 have a rectangular cross-section and are best be described by a hard wall potential. However, this approach does not yield an analytical solution and we therefore use the approximation of a parabolic wall potential $\frac{K}{2} z^{2}(x)$ as illustrated in figure 6.2 a). Here, $K$ is the constant which determines the strength of the channel wall potential. To describe a confined worm-like chain, we need to account for the wall potential in the Hamiltonian in equation 6.3. With the assumption of the parabolic wall potential we obtain

$$
\mathcal{H}_{c}[z(x)]=\int_{0}^{L_{x}} d x\left[\frac{\kappa}{2}\left(\frac{\partial^{2} z(x)}{\partial x^{2}}\right)^{2}+\frac{K}{2} z(x)^{2}\right] .
$$




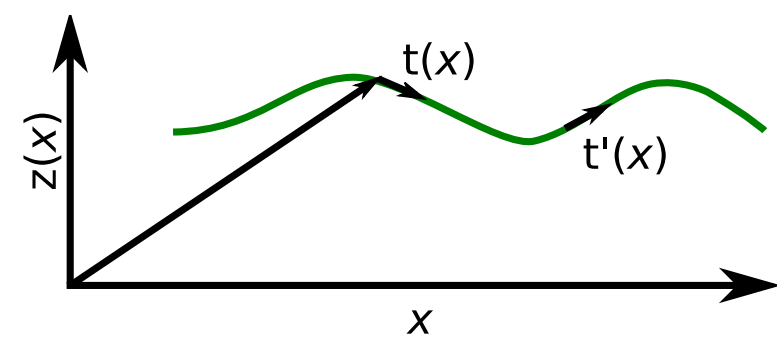

Figure 6.3: Monge parametrization.

This Hamiltonian assumes that the bending energies in the $x-y$ plane and the $x-z$ plane decouple. Because the filament is oriented along the main direction of the channel, we benefit from using the so called Monge parametrization as illustrated in figure 6.3. Because we consider a 2 dimensional model, we use the scalar value $z(x)$ instead of the vector $\vec{z}(x)$. Also, we introduce the deflection length [51]

$$
\lambda=\sqrt{2}\left(\frac{\kappa}{K}\right)^{\frac{1}{4}} .
$$

This length scale is defined by the competition of both bending stiffness and the strength of the confining potential. $\lambda$ corresponds to the average distance between two points, where the filament touches the channel walls as illustrated in figure 6.4. Also, the deflection length connects the channel width $d$ and the persistence length $L_{P}$ in a scaling law formulated by Odijk in 1983 [51]:

$$
\lambda=a d^{\frac{2}{3}} L_{P}^{\frac{1}{3}}
$$

From here on, we will refer to this scaling law as Odijk's scaling law. Here, $d$ is the channel width and $a$ is a coefficient dependent on the channel geometry. Thus, the value of $a$ remains constant for identical geometries for ideal worm-like chains. We later use this property to compare different systems of worm-like chain polymers.

With the inclusion of the deflection length $\lambda$ and the assumption of Boltzmann distributed energies for the filament system, we can formulate a tangent correlation function for confined filaments 36

$$
\langle\cos \theta(l)\rangle=1-\frac{\lambda}{2 \sqrt{2} L_{P}}\left(\cos \left(\frac{\pi}{4}\right)-\cos \left(\frac{l}{\lambda}+\frac{\pi}{4}\right) \exp \left(\frac{-l}{\lambda}\right)\right) .
$$

Here, $l$ is the distance between two tangents according to Monge parametrization. The tangent correlation function contains two fit parameters that characterize a worm-like chain filament in a confining channel geometry. The parameters are the persistence length $L_{P}$ and the deflection length $\lambda$. 


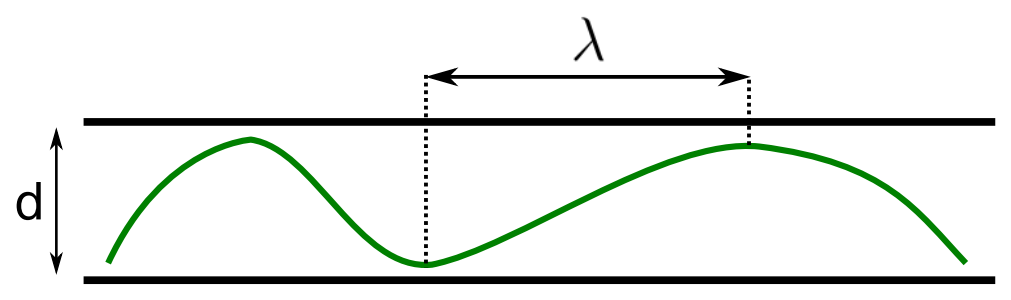

Figure 6.4: Illustration of the deflection length $\lambda$.

When we compare the tangent correlation functions for both the free and confined case, we find a marked difference for long correlation distances. In the free case, described by equation 6.4, the correlation function decays to zero for large $l$ - correlation is lost. In contrast, the correlation function for confined filaments in equation 6.9 converges to a constant value which depends only on the channel geometry and the mechanical properties of the filament:

$$
\langle\cos \theta\rangle_{\infty} \approx 1-\frac{\lambda}{4 L_{P}} .
$$

While the theoretical description we have described above certainly has strong merit, we have to be aware of some limitations. First, the analytical solution shown above does not account for the hard wall potential found in our experiments. However, Monte-Carlo simulations performed by Köster et al. have shown that the behavior of a filament remains largely unaffected by this change. Secondly, the tangent correlation function assumes infinitely long filaments - end-effects are neglected. The resulting requirements for experimental data are discussed in detail in chapter 6.2.1.

\subsubsection{Odijk's scaling law}

In chapter 6.1.2 we have introduced the Odijk's scaling law for worm-like chains in strong confinement

$$
\lambda=a d^{\frac{2}{3}} L_{P}^{\frac{1}{3}}
$$

This scaling law gives a relation between the deflection length, the channel width and the persistence length of a given system. By choosing different channel widths $d$ we aim to test the scaling law for consistency. However, because the persistence length $L_{P}$ is a material property, we have only access to one fixed value for vimentin filaments. Therefore, we include data for a second, semi-flexible polymer, the actin filament.

The strong confinement, which defines the regime in which Odijk's scaling law can be applied is defined by basic conditions relating to the typical length scales of 


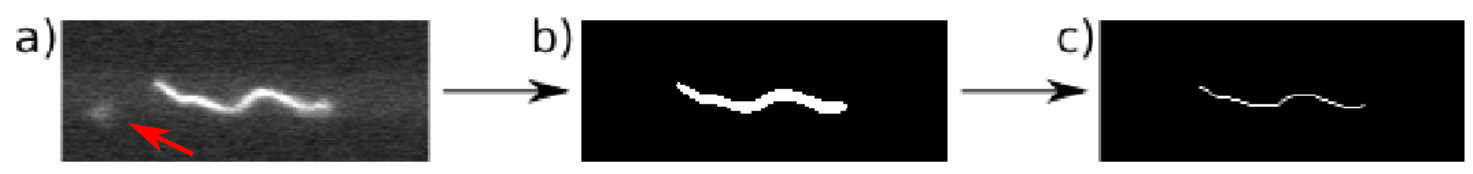

Figure 6.5: Image processing steps a) Original fluorescence microscopy image. Red arrow indicates a fluorescent artifact not connected to the filament. b) After application of a thresholding algorithm and removal of artifacts we obtain a binary image of the recorded fluorescence. c) A one pixel line is calculated by means of a thinning algorithm.

the system. The conditions are as follows:

$$
L \gg L_{P} \gg d
$$

Vimentin filaments satisfy the condition $L \gg L_{P}$ very well, as we are able to assemble filaments with lengths of $30 \mu \mathrm{m}$ and more and we can assume $L_{P} \approx 1 \mu \mathrm{m}$. However, channels with widths $d \ll 1 \mu \mathrm{m}$ can not be produced using soft-lithography techniques. Also, for channels with widths below $1 \mu \mathrm{m}$ the analysis of the filament contour through a tangent-tangent correlation becomes less reliable because of the resolution limit of fluorescence microscopy. When we use vimentin filaments, we can therefore only fabricate an experimental setup, where $L_{P} \approx d$. In contrast, for actin filaments the persistence length is on the order of the filament length $L \approx L_{P}$. In turn, for actin filaments $L_{P} \gg d$ is satisfied. Consequently, in combination both filament systems promise to give a comprehensive validation of the scaling law by means of a comparison of the scaling constant $a$.

\subsection{Analysis}

For data analysis we use both MatLab (MathWorks, Natick, Massachusetts, United States) and ImageJ scripts. At first, we need to obtain a contour that we can analyze with the theoretical framework provided in chapter 6.1. To this end, the fluorescence microscopy gray-scale image is first converted into a binary image using a thresholding algorithm provided by ImageJ. Importantly, the algorithm accounts for fluctuations of the image intensity throughout an image sequence and thus enables us to analyze images independent of fluorescence bleaching processes. Artifacts are removed before further processing the image, as illustrated in figure 6.5. The last step of the initial image preparation process is the reduction of the "filament" outline to a single one-pixel-line. We achieve this by applying a thinning filter that removes individual pixels until only the desired one-pixel-line remains. An illustration of the image processing procedure can be found in figure 6.5 . 
As an alternative to the image analysis using our own methods, a pre-made solution in the form of the java program "jfilament" 94 has been used to obtain filament contours. Results obtained with this program and our own methods are in very good agreement. However, "jfilament" uses a minimal energy assumption for the determination of the filament contours, which might have some influence on our results. Therefore, we chose to use an image analysis system without physical constraints to obtain unbiased data.

Importantly, the analysis of the filament contours is time independent, which can be seen on the tangent-correlation function in equation 6.9. The time interval between two individual images is therefore of little consequence, and also varying intervals can be accepted. Thus, individual images from an image sequence can be discarded without compromising the data analysis process, when they do not yield satisfactory one-pixel lines. A brief discussion of the timing, the minimal amount and quality of filament images necessary to obtain authentic data from a single filament follows in chapter 6.2.1.

The one pixel line calculated from the fluorescence image has only discreet values and thus has to be further processed. We apply a cubic smoothing spline, where all values of the contour are included in the resulting spline. From these splines, a tangent correlation function is computed in a MatLab script. In this step, we compute the weighted spatial average over the length of the filament as well as a temporal average over an image series of an individual filament.

As a final step, we fit the resulting tangent correlation function with an algorithm written by Jan Kierfeld [95]. We can thus calculate the free fitting parameters $\lambda$ and $L_{P}$. Because we analyze the projected contours, using a two dimensional worm-like chain model, we obtain the intrinsic persistence length of the filaments. As stated before, the persistence length is a material property, which mostly remains unaffected by changes to the experimental system.

\subsubsection{Statistical considerations}

To ensure that our data analysis leads to meaningful results we need to carefully consider, which filaments we can analyze and in what manner we can improve our statistics. Important parameters, such as the recording time and the filament length can, to some extend, be controlled experimentally. Also, the calculation of a statistical average has to be performed sensibly.

We begin with the parameters that we can influence experimentally. The 
length of the filaments can, unfortunately, not be specified directly through experimental conditions as it is possible for DNA. We can only produce a filament solution, where the length distribution is influenced by assembly time and temperature as well as protein concentration. Consequently, we record data from filaments of different lengths. Depending on the channel width, effects of decreasing confinement of the filament ends begin to manifest at a certain lower filament length. By only considering filaments with a minimal length of $10 \mu \mathrm{m}$, this problem can largely be avoided. If we want to observe filaments in channels wider than $2.7 \mu \mathrm{m}$, we need to adjust the minimal filament length accordingly.

As already mentioned above, there is also a minimal total observation time and image number that leads to reasonably good statistics for a tangent correlation function of a single filament. In reference [96] we have conducted several tests that help us to determine imaging parameters. By using a single image series and splitting it into individual, shorter image series, we have found that a minimal total observation time of 15 seconds is reasonable. By recording image series at least twice as long, we have a buffer of images that we can discard and still meet our quality standards. Lastly, we need to determine the minimal number of contours of an individual filament that we need for a meaningful result. By considering a single image series of 600 frames and only using every n-th frame, we find that at least 100 images are necessary to obtain a high quality tangent correlation function for the individual filament.

Alternatively to recording an image sequence of an individual filament, it is possible to record several images of different filaments and process them as described above. However, the analysis of image sequences has the advantage that we can easily detect unwanted experimental influences, such as flow in the channel or a filament that has adsorbed to a surface.

Now that we have set the framework for the recording and analysis of an individual image series, we consider the averaging of many different image series. The statistical weight that a correlation value $\left\langle\cos \left(\theta\left(l_{i}\right)\right\rangle\right.$ has depends on two parameters. Firstly, for a filament with a given contour length $L$, many values contribute to the average correlation for $l \ll L$ and only few values contribute for $l \lesssim L$. Secondly, when we compare filaments of different lengths, two identical correlation distances have a different statistical weight in each of the correlation functions. When averaging filaments of different lengths we take this into account by using a weighting factor that scales with respect to the filament length. 

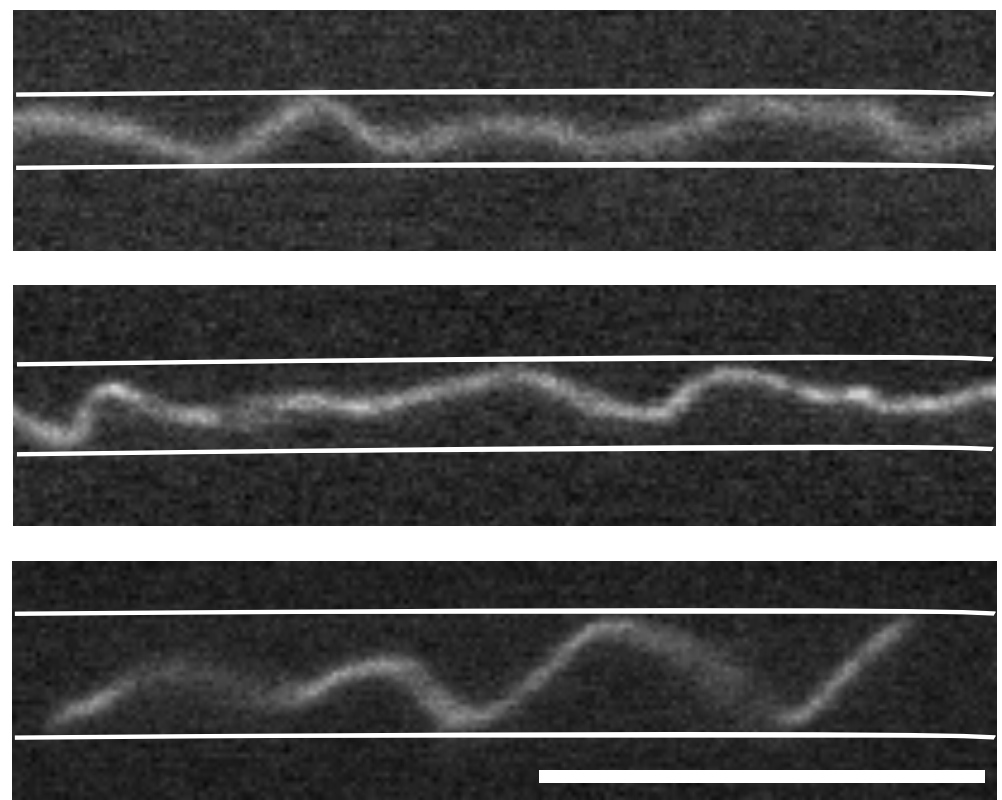

Figure 6.6: Fluorescence images of vimentin filaments in channels of different widths. The confinement of the filaments decreases from the narrowest channel (white lines) with a width of $1.2 \mu \mathrm{m}$ over the middle channel with a width of $1.6 \mu \mathrm{m}$ to the widest channel with a width of $2.7 \mu \mathrm{m}$. The characteristic fluctuations of the filaments change with the channel width. The channel height is $1 \mu \mathrm{m}$ in all cases. Scale bar $10 \mu \mathrm{m}$.

\subsection{Results}

With the groundwork of chapters 6.1 and 6.2 we are now equipped to evaluate the raw data we have obtained. Here, we show which influence different channel geometries have on the filament's behavior and the consequences this behavior has with respect to the worm-like chain model. Also, we are able to determine the basic mechanical properties of the filaments. At this point it is important to note that the filaments used in these experiments were exclusively assembled in dialysis tubing. This ensures that we obtain filaments with a uniform number of monomers per cross-section 9,12 . Consequently, cross-section polymorphism has no influence on our measurements.

We have analyzed a total of more than 50000 images, which amounts to 20 - 70 individual filaments for each of the 6 different channel geometries listed in table 6.1 . These data provide a very solid basis for further analysis with the aim of confirming Odijk's scaling law. 


\begin{tabular}{c||cc} 
geometry & width $[\mu \mathrm{m}]$ & height $[\mu \mathrm{m}]$ \\
\hline \hline 1 & 1.2 & 0.45 \\
2 & 1.6 & 0.45 \\
3 & 2.7 & 0.45 \\
4 & 1.2 & 1 \\
5 & 1.6 & 1 \\
6 & 2.7 & 1
\end{tabular}

Table 6.1: The channel geometries used in our experiments By choosing widths and heights differing at least by a factor of two, we can observe distinctly different filament behavior as shown in figure 6.6 .

\subsubsection{Effects of changing channel width}

As shown in image 6.6 even a small differences in channel width, here an increase of about a factor of 2 , have a strong impact on the resulting filament fluctuations. While a filament in a channel with a width of $d=1.2 \mu \mathrm{m}$ is rather strongly confined and thus assumes a relatively straight contour, the filament in a channel with a width of $d=2.7 \mu \mathrm{m}$ is notably more free in its movement and consequently looks more undulating.

From the analysis method described in chapter 6.2 we obtain the tangent correlation functions shown in figure 6.7. The tangent correlation function begins with a decay, which is similar to that of an unconfined filament. Depending on the grade of confinement, this initial decay is very brief for narrow channels and more pronounced for wider channels. For long correlation distances, the tangent correlation function approaches a constant value as indicated by equation 6.10. Again, a narrower channel results in an increased overall correlation. For wider channels we observe effects of the finite filament length, the tangent correlation function shows a slight decay. The error of the individual curves is the standard deviation of the mean. Additionally, we show the fit of the tangent correlation functions with the analytical solution for confined filaments in a parabolic potential given by equation 6.9 .

\subsubsection{Effects of changing channel height}

As described in chapter 6.1.2 with equation 6.6 the motions of an ideal worm-like chain in the $x-y$ plane and the $x-z$ plane are expected to decouple. Therefore, 


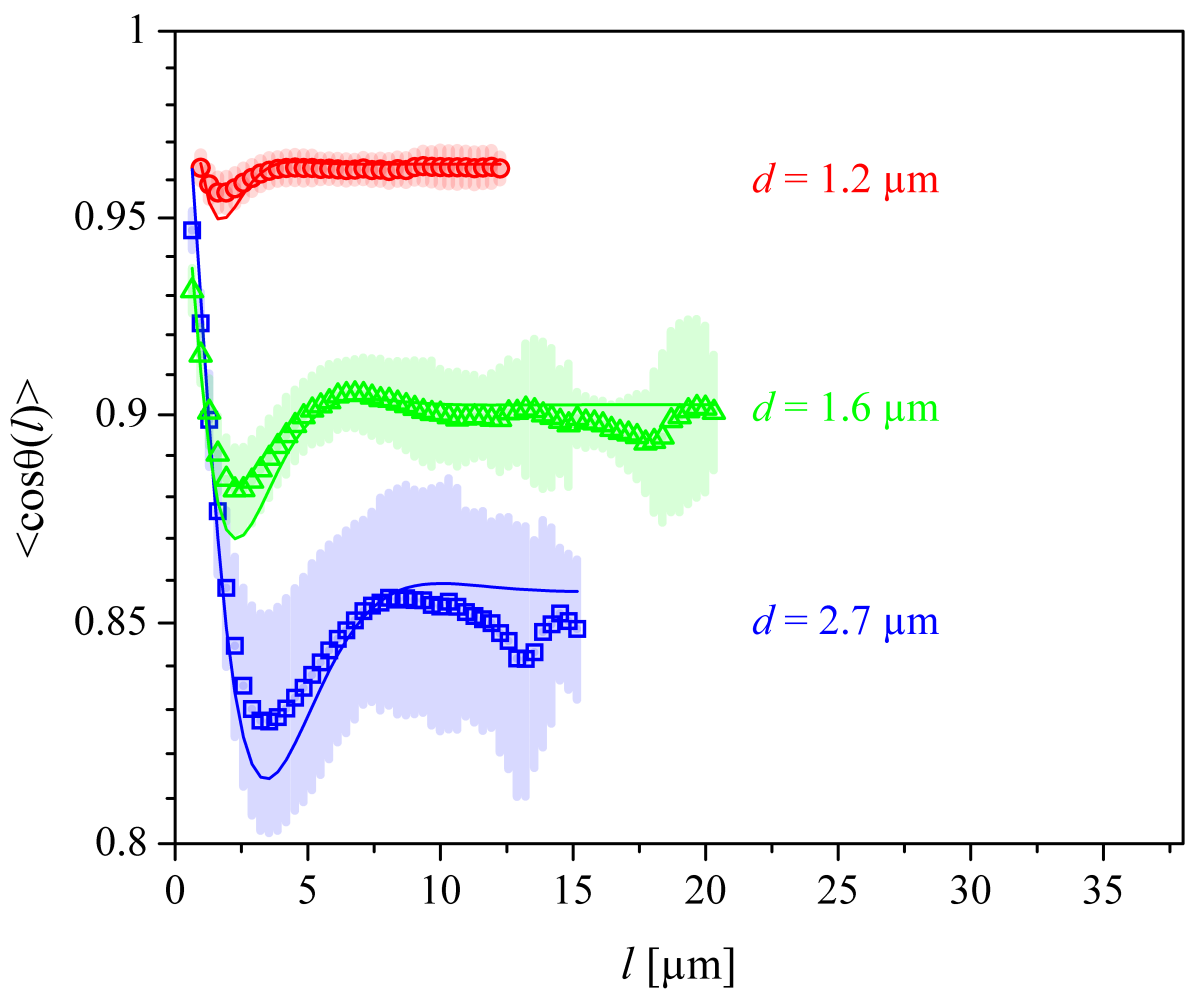

Figure 6.7: Tangent correlation functions for filaments in channels of different widths. Data for each curve are obtained by averaging over at least 20 individual filaments. The error of the mean value is shown in a lighter color and increases with decreasing confinement. Each curve is fitted with the analytical solution for confined filaments. The channel height for all curves is $h=1 \mu \mathrm{m}$.

a change in channel height has no influence on the projection of an ideal worm-like chain that is observed with fluorescence microscopy techniques. We use our experimental data from vimentin filaments in channels of different heights to verify this assumption. To this end, we compare experiments with different channel heights by means of the respective tangent correlation functions. Changes in channel height and channel width should have similar effects on the filaments fluctuations. Therefore, we can assume that a change in channel height by a factor of 2 yields a notably different behavior, if it is indeed present. We have acquired data for filaments in channels with a height of $h=0.45 \mu \mathrm{m}$ in addition to the data for filaments in channels with a height of $1 \mu \mathrm{m}$. The resulting tangent correlation functions are plotted in figure 6.8 together with the functions from figure 6.7. The tangent correlation function pairs for filaments confined in channels of the same width but different 


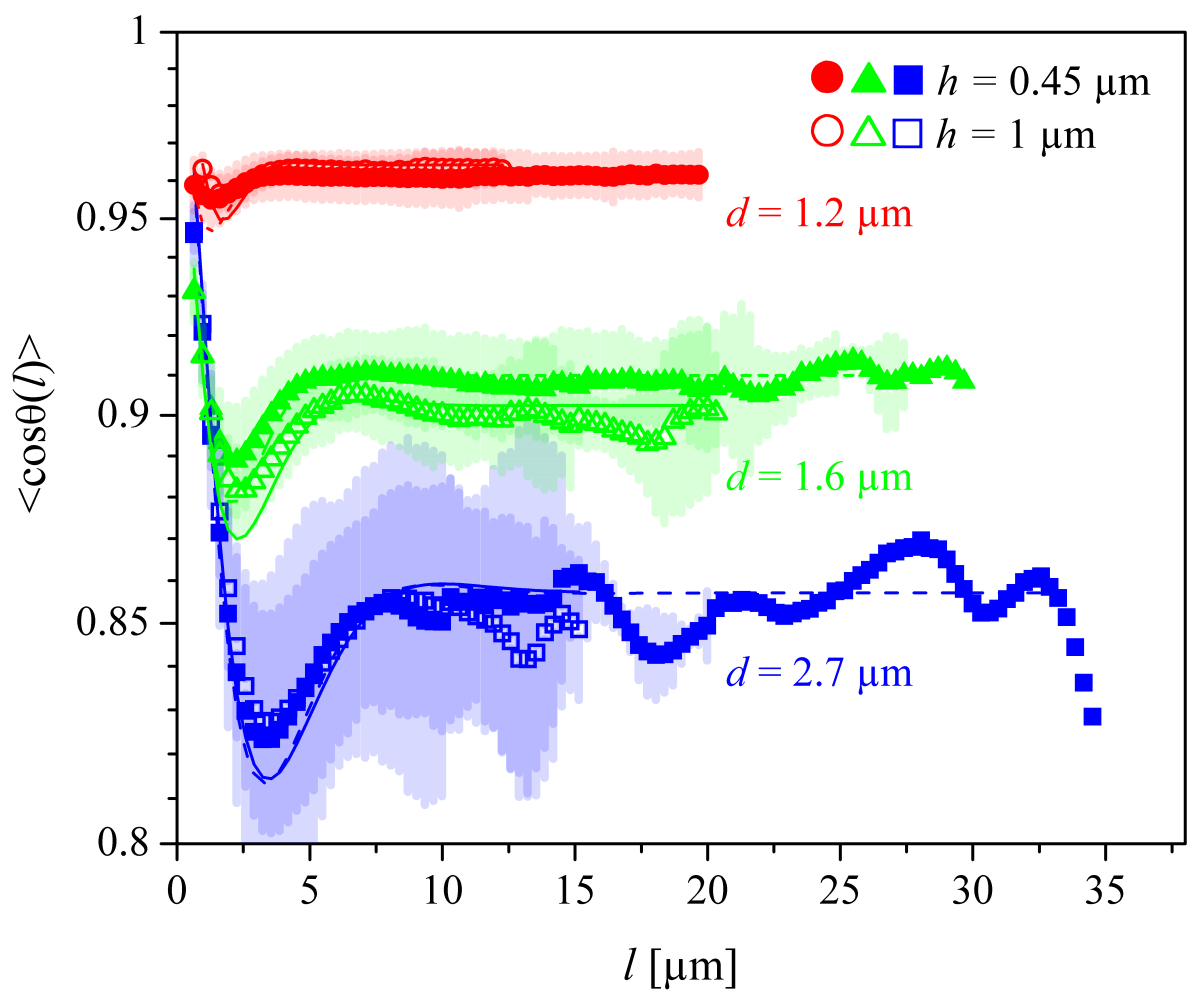

Figure 6.8: Pairs of tangent correlation functions for filaments in channels of equal widths but different heights. Each curve pair shows identical behavior. Dashed lines show analytical fit for the data from channels with heights of $h=0.45 \mu \mathrm{m}$.

heights show identical behavior. Motions in perpendicular directions decouple for vimentin filaments. In this regard, vimentin filaments behave identical to an ideal worm-like chain.

\subsubsection{Tangent-correlation fits}

After comparing the properties of filaments in channels with different confinement geometries, we can now consider the fits of the data with equation 6.9. We obtain values for both the persistence length $L_{P}$ and the deflection length $\lambda$ of the vimentin filaments. The values are plotted in figure 6.9 for each channel geometry. While the deflection length is specific to the channel geometry used in the respective experiments, and is expected to increase with the channel width, the persistence length is a universal material property. By combining data for $L_{P}$ from all measurements, we obtain a mean value for the persistence length of vimentin intermediate filaments in 

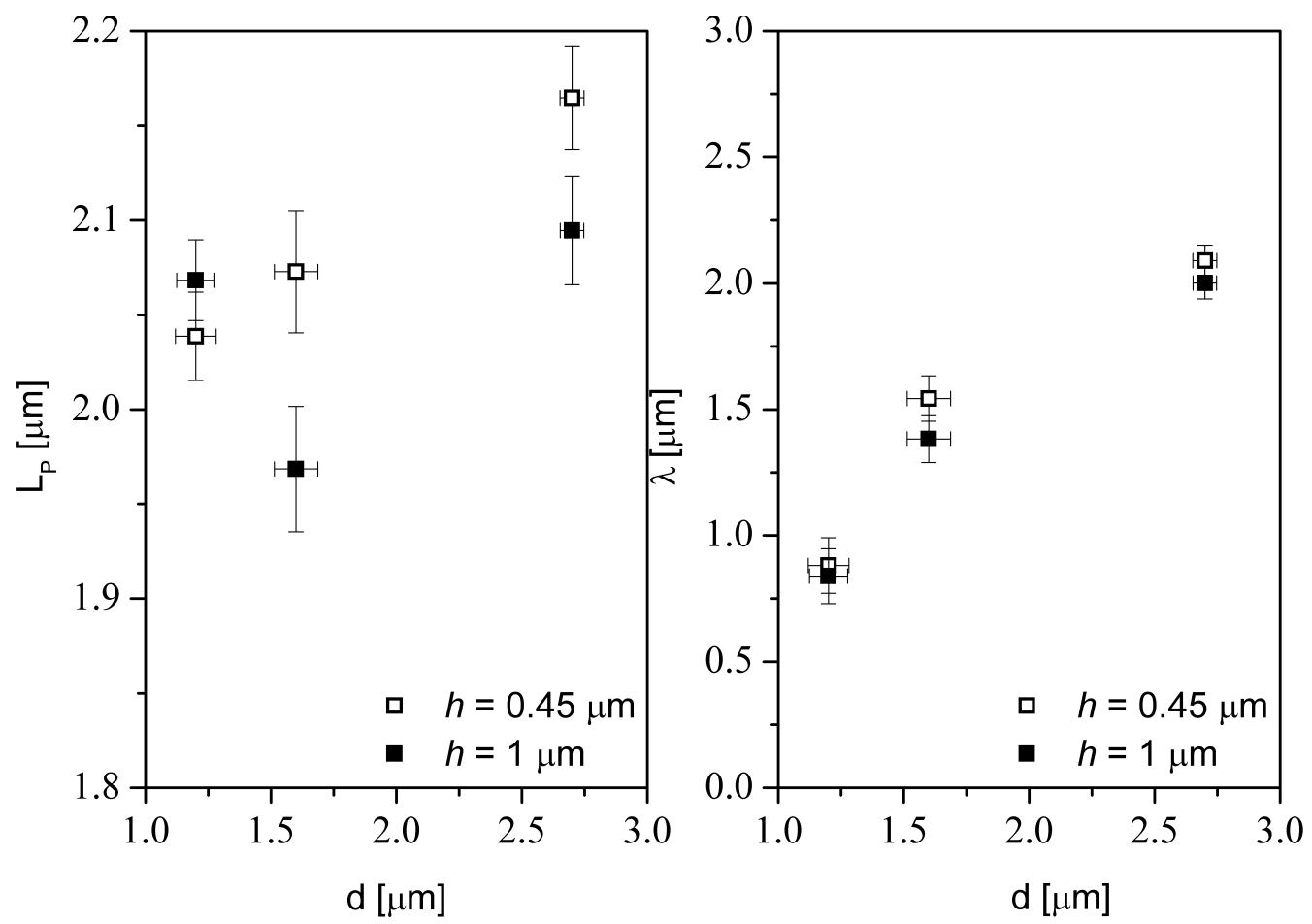

Figure 6.9: Persistence length $L_{P}$ and deflection length $\lambda$ from fits. Results for the persistence length remain constant for different channel geometries. Values for deflection length $\lambda$ increase with the channel width but remain independent of channel height.

solution:

$$
L_{P}=2.1 \pm 0.1 \mu \mathrm{m}
$$

Measurements on filaments restricted between two glass cover slips yield a similar value of $L_{P \text { (unconf.) }}=2.0 \pm 0.5 \mu \mathrm{m}$, which is in very good agreement with our measurements on confined filaments.

\subsubsection{Application to a scaling law}

In chapter 6.1 .2 we have introduced Odijk's scaling law

$$
\lambda=a d^{\frac{2}{3}} L_{P}^{\frac{1}{3}}
$$

With the values for the deflection length $\lambda$ and the persistence length $L_{P}$ from chapter 6.3 .3 we confirm this relation with experimental data. To this end, we use the scaling constant $a$ as a means for comparison. As discussed in chapter 6.1 .3 we use 


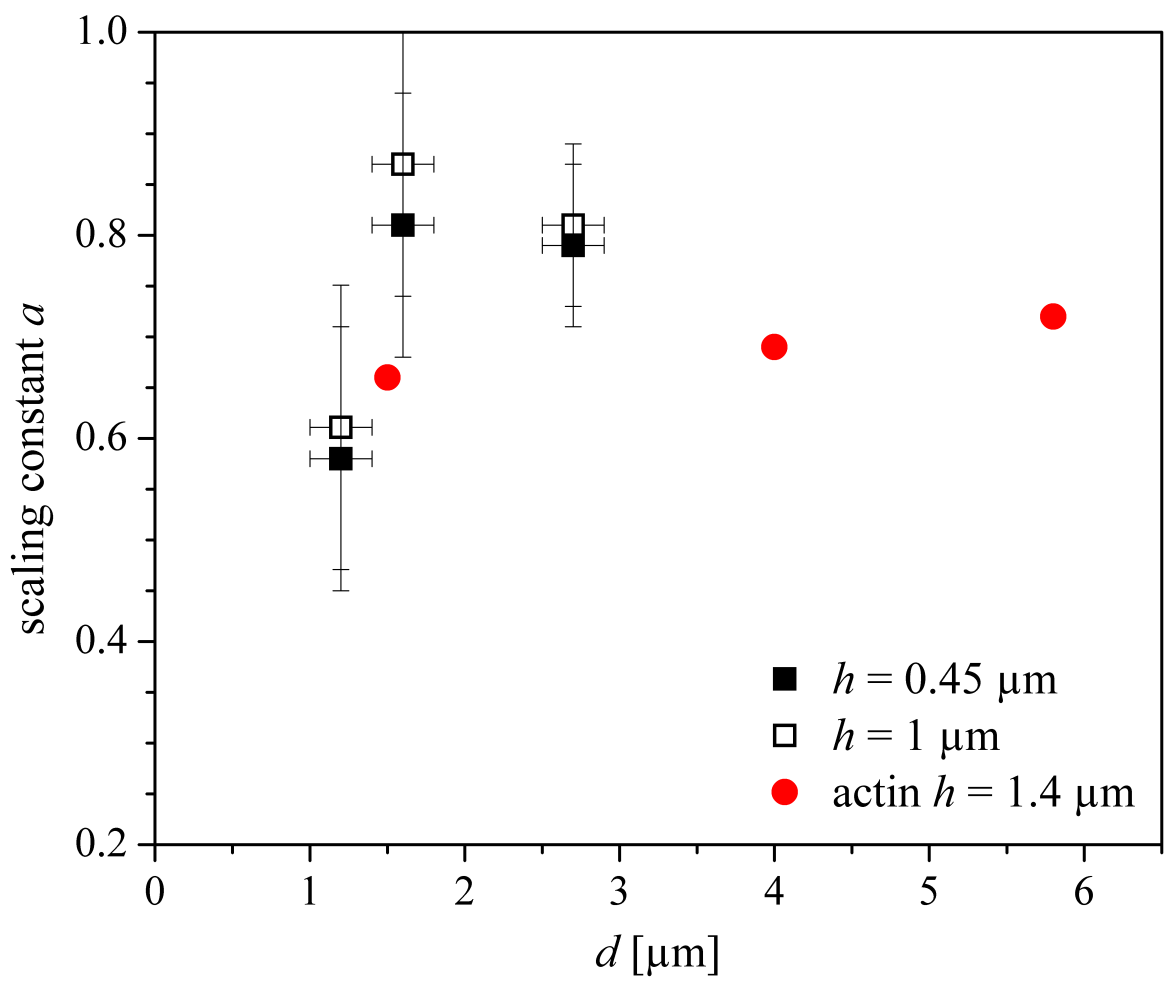

Figure 6.10: Comparison of the vimentin and actin filament systems by means of the scaling constant $a$. Inclusion of actin data from 91] shows that the scaling constant $a$ is very similar for the two filament systems. Differences in the channel dimensions have no impact on the scaling constant.

data from measurements on actin filaments to gain a second value for the persistence length $L_{P}$. This comparison is especially compelling, since actin and vimentin filaments do not only have different persistence lengths, but also have markedly different molecular structures. Vimentin consists of rod-like subunits assembled in a hierarchical manner. In contrast, actin filaments consist of globular subunits which assemble in an ATP-dependent process into a double-helical filament.

We plot the values for the scaling parameter $a$ for actin and vimentin in figure 6.10. For vimentin filaments, we obtain 6 distinct values for the three channel widths in two different heights. Data for actin filaments are also available for wider channels. Both data sets have a certain overlap in terms of channel width. We find that the values for the scaling constant $a$ of the two experimental systems agree very well. The scaling law proposed by Odijk in the 1980s [51] is thus nicely confirmed. 


\subsection{Discussion}

The results from our experiments on confined vimentin filaments can be roughly separated into three categories. Firstly, we have measured the persistence length of the filaments. Here, we discuss our findings in the context of other measurements of the persistence length of vimentin filaments. Also, we consider the relevance of our findings for the mechanical properties of other intermediate filaments. Secondly, we discuss the impact of the general polymer physical results we have obtained. The third observation is that by employing narrow channels, we strongly improve our measurements. We discuss this finding in the context of the configuration space the filaments can access.

\subsubsection{Persistence length of vimentin}

The persistence length of different intermediate filaments has been measured in the past using different methods. We start the discussion of our results by a comparison with measurements of individual vimentin filaments adsorbed to a surface using an atomic force microscope (AFM) by Mücke et al. [33]. They find apparent persistence lengths for vimentin filaments which are dependent on the substrate type. As shown in figure 6.11 the apparent persistence length increases from mica $\left(L_{P, a p p}=0.337 \mu \mathrm{m}\right)$ over HOPG (highly oriented pyrolytic graphite, $\left.L_{P, a p p}=0.675 \mu \mathrm{m}\right)$ to hydrophillic glass $\left(L_{P, a p p}=1.061 \mu \mathrm{m}\right)$. It is therefore clear, that the substrate has some influence on the apparent mechanical properties of the filament system. Mücke et al. argue that, with basic assumptions on the substrate properties, a persistence length of vimentin filaments of $1 \mu \mathrm{m}$ to $1.3 \mu \mathrm{m}$ is likely.

The concepts of the experiments performed by Mücke et al. and by us are basically similar. The contour of the filament is analyzed to obtain the mechanical properties of the filament. The major difference between both approaches is the amount of interaction with a substrate. In the case of filaments in confinement (or freely fluctuating filaments) interaction with a surface is avoided. Additionally, experiments on freely fluctuating and confined filaments performed by us are in good agreement. We therefore assume that the value of the persistence length measured by us is the true material property of the filament instead of the apparent value measured on adsorbed filaments.

Apart from measurements on individual filaments, there have been measurements of the persistence length of vimentin filaments in bulk solution. Mechanical 

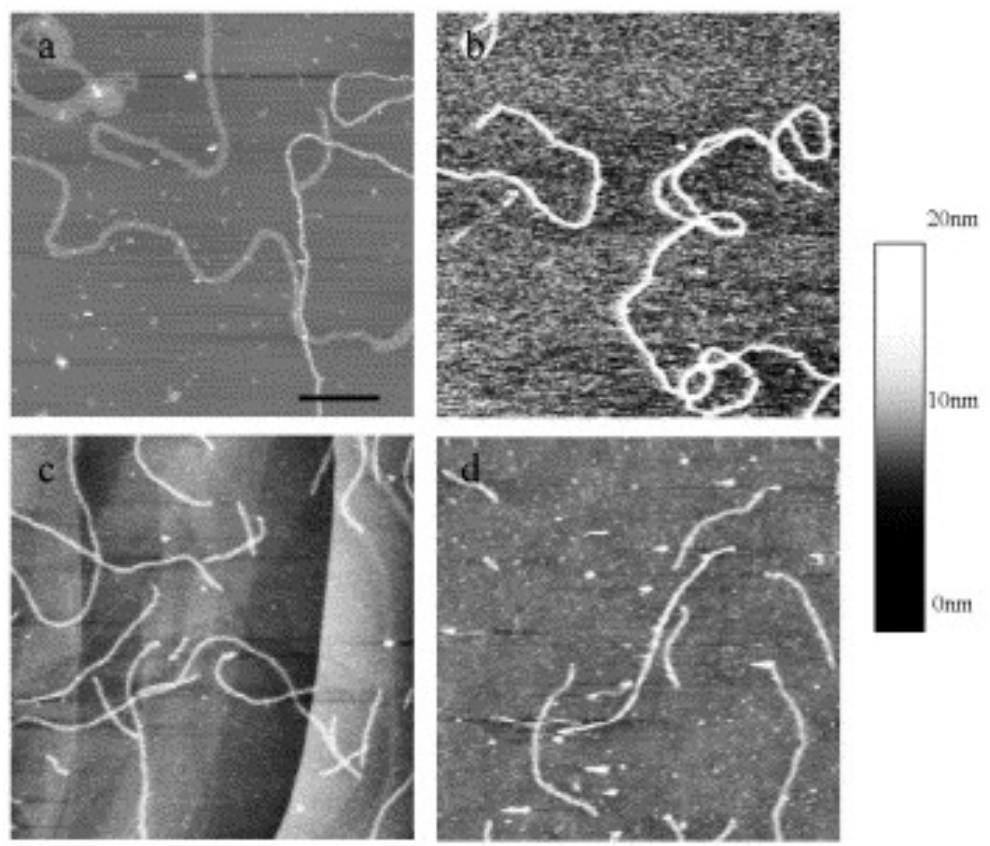

Figure 6.11: Vimentin filaments adsorbed on different substrates. Filaments are imaged using AFM methods on a) mica in air b) mica in water c) HOPG d) hydrophillic glass. The filaments assume different contours on different substrates. On mica they are relatively tightly wound, whereas they are more or less straight on a glass substrate. Scale bar $1000 \mathrm{~nm}$. With permission from 33.

rheometry experiments by Schopferer et al. give values for the persistence length of $L_{P, r h e} \approx 0.4 \mu \mathrm{m} \mathrm{[32]}$. In this case the experimental procedure makes it necessary to assemble vimentin filaments at a relatively high concentration of $1 \mathrm{~g} / \mathrm{L}$, which is five times the concentration routinely used by us and others to assemble filaments. Also, the measurement itself takes place at comparatively high protein concentrations, which might lead to further, unpredictable interactions between the filaments, which are unrelated to their actual mechanical properties. The different assembly conditions might affect the properties of the filaments in the bulk filament solution through the formation of an entangled network or similar interactions of the filaments with themselves. Therefore, comparing the results of this study with our own is somewhat difficult and we are content to note that despite strong experimental differences we measure similar mechanical properties of vimentin filaments.

After comparing our results with two different studies on vimentin filaments, we also include data for non intermediate filament polymers. Here, we exploit the fact that a vimentin filament basically consists of $16 \alpha$-helical coiled-coils - the vimentin dimer shown in figure 2.1. By looking at the properties of a single such $\alpha$-helical coiled-coil filament we can extrapolate the properties of vimentin filaments 
and compare the results to our measurements.

An entirely unrelated filamentous protein with a $\alpha$-helical coiled-coil structure is the tropomyosin strand, found in smooth muscle. As Sousa et al. show from the analysis of electron micrographs, a tropomyosin strand has a persistence length of about $100 \mathrm{~nm}$ [97]. Similar values are found for light meromyosin, which is also a coiled-coil filament, by Hvidt et al. via rheology experiments [98. Finally, simulations of the most basic plausible structure of a $\alpha$-helical coiled-coil give a persistence length of about $150 \mathrm{~nm}$ [99]. These values, found with different techniques, allow us to assume a persistence length for $\alpha$-helical coiled-coils in the range of 100 to $150 \mathrm{~nm}$.

To compare the persistence length of these individual coiled-coils with that of the vimentin filament, we calculate the persistence length of a bundle of such coiled-coils. In general, the persistence length of a bundle of individual filaments can be calculated to 100

$$
L_{P, \text { bundle }}=N^{\gamma} L_{P, \text { filament }} .
$$

Here, $\gamma$ is the so called coupling factor. Values for $\gamma$ are in the region of $1 \leq \gamma \leq 2$. A value of $\gamma=1$ describes filaments that form an entirely uncoupled bundle. The filaments can move freely along the direction of the bundle. When the individual filaments are fully coupled, we find $\gamma=2$. If we now assume that the $16 \alpha$-helical coiled-coils in the vimentin cross-section are not cross-linked at all, we estimate the persistence length of the vimentin filament with the equation

$$
L_{P, \text { uncoupled }}=N L_{P, c c} \text {. }
$$

Here, $L_{P \text {,uncoupled }}$ is the persistence length of the vimentin filament and $L_{P, c c}$ is the persistence length of an individual coiled-coil. With the values for the persistence length for $\alpha$-helical coiled-coils found above $\left(L_{P}=0.1 \mu \mathrm{m}\right.$ to $\left.0.15 \mu \mathrm{m}\right)$, we expect a persistence length of roughly $1.5 \mu \mathrm{m}$ to $2.5 \mu \mathrm{m}$ for vimentin filaments. The values for $L_{P, \text { uncoupled }}$ are very similar to our results for vimentin filaments in confinement. Of course, we have not taken into account a possible strong coupling between the individual vimentin dimers. In the case of fully coupled coiled-coils, we can calculate the persistence length of the filament with the equation

$$
L_{P, \text { coupled }}=N^{2} L_{P, c c} .
$$

This gives values between $25 \mu \mathrm{m}$ and $38 \mu \mathrm{m}$, which are clearly far larger than the persistence lengths typically observed for intermediate filaments. Nonetheless, this 
estimate is useful for the purpose of comparison of existing measurements, especially between different intermediate filament types.

In short, a single coiled-coil filament has a persistence length of $0.10 \mu \mathrm{m}$ to $0.15 \mu \mathrm{m}[97-99]$. Therefore, a filament consisting of 16 of these coiled-coils would have a persistence length between $L_{P, \min }=1.6 \mu \mathrm{m}$ for totally uncoupled coiled-coils and $L_{P, \max }=38 \mu \mathrm{m}$ for totally coupled coiled-coils. A value near the lower limit for an arrangement of 16 coiled-coils is an excellent match with our measurements. However, this also implies a very limited coupling between the constituents of the filament. A closer look at the structure of the vimentin dimer reveals that we do, in fact, not deal with a perfect coiled-coil. Two linker regions, visible in figure 2.1, disrupt the coiled-coil and might be responsible for more flexibility in the vimentin dimer than that of an ideal coiled-coil. Thus, it is difficult to determine the exact coupling properties. When we estimate a lower value for the persistence length of a dimer $L_{P, c c}=0.1 \mu \mathrm{m}$ and use the value for the persistence length of vimentin we have obtained from our measurements $L_{P, \text { vimentin }}=2.1 \mu \mathrm{m}$, we obtain a coupling factor of

$$
\gamma_{\text {vimentin }} \approx 1.1
$$

The weak coupling between the subunits of intermediate filaments most likely is part of the reason for the high flexibility of the filaments under strain. The dimers are able to slide with respect to each other and thus elongate. A significant reduction in filament diameter observed by Kreplak et al. supports this idea [31].

The persistence length we measure for vimentin filaments can also be useful to extrapolate the general properties of other intermediate filaments. Here, it is likely that the intermediate filaments sharing the most characteristics have similar properties. Both desmin and vimentin are Type III intermediate filaments and are able to co-assemble into filaments. Therefore, we expect similar mechanical properties for vimentin and desmin. Desmin filaments have a persistence length of $L_{P, r h e} \approx 0.9 \mu \mathrm{m}$, which was measured using rheometry methods [32]. Because of the very similar structure of both filaments, it is reasonable to assume that a measurement of individual desmin filaments in solution would result in an increased value, similar to that found by us for vimentin. For other intermediate filament types such as keratins, where only 8 dimers per cross-section form a filament we expect a lower persistence length than for vimentin, which has a cross-section of 16 dimers. And in fact, measurements by Lichtenstern et al., which are similar to those performed by Mücke et al. give a persistence length of $300 \mathrm{~nm}$ for keratin K8/K18, 
which is about a third of the value obtained for vimentin with the same method [34.

The difference in persistence length between filament types with a different number of dimers in their cross-section can also be used to calculate the coupling factor $\gamma$ in the equation 6.15. Here we use the difference in persistence lengths as measured on surfaces 33,34 between vimentin and keratin K8/K18 by a factor of $L_{P, \text { vimentin }} / L_{P, \text { keratin }} \approx 3.3$. By using the different number of dimers in both filament types, we are able to calculate an estimate of the coupling factor. We obtain the value

$$
\gamma_{I F} \approx 1.6
$$

which indicates a coupling of the dimers of intermediate strength. Of course, we have to assume that the interaction between the dimers in both keratin and vimentin filaments is identical for our estimation to be strictly true. Thus, we can only take this coupling factor as an indicator for the actual circumstances in intermediate filaments. Also, we can use it to calculate an estimate of the persistence length of the dimeric subunits in the vimentin filament by inserting the coupling factor $\gamma_{I F} \approx 1.6$ and the persistence length of the filament $L_{P, \text { vimentin }}=2.1 \mu \mathrm{m}$ in equation 6.15 . We thus obtain a persistence length for dimeric vimentin $L_{P, \text { dimer }} \approx 0.25 \mu \mathrm{m}$. This value is far below that of other $\alpha$-coiled-coils as listed above. Nonetheless, this comparison leads in the right direction and detailed experiments on the persistence length of dimeric vimentin (or other intermediate filaments) could yield further insights into the coupling factor in filaments and thus give further insights into the molecular bonds between the dimers that make up the intermediate filaments.

\subsubsection{Properties of confined filaments}

With data collected from vimentin filaments in confining microchannels and the inclusion of actin filament data, we are able to verify Odijk's scaling law formulated in the 1980s. The two very different polymer systems are both described very well by the worm-like chain model. This is quite remarkable, since the worm-like chain model itself is very straightforward in terms of basic constraints and assumptions. Also, we are able to confirm that the fluctuations of the filaments in perpendicular directions do, in fact, decouple. This result is of great importance, because otherwise the observation of the filaments projection with fluorescence microscopy would only yield incomplete results.

The confining channel geometry used in our experiments is in a certain manner an approximation of the situation in the cell, which is a very crowded system. The 

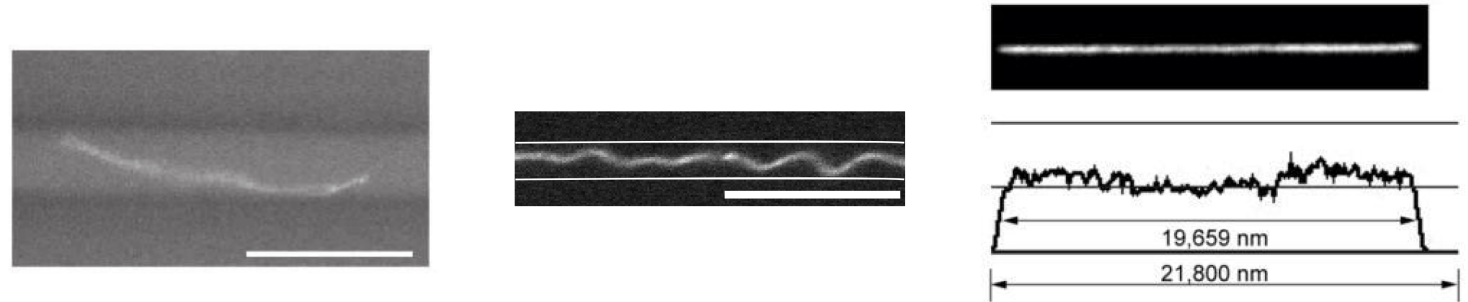

Figure 6.12: Different bio-polymers confined in nanochannels: From left to right: actin, vimentin and DNA with intensity profile. For both actin and vimentin the filament contour is clearly visible. The characteristic wavelengths of the fluctuations are clearly different. The persistence length of DNA is so low that the contour can not be resolved with normal microscopy methods. Scale bars show $10 \mu \mathrm{m}$. Actin and DNA images with permission from [36] and [48].

lack of free space in the cell results in a natural confinement of the filamentous systems to channel like tubes. Two main differences between the cellular system and our own microfluidic device are apparent. Firstly, our channels are straight, and width as well as height are constant over time, whereas the cellular environment produces channels of varying curvature and cross-section. The change in channel geometry is reflected in an adapted Hamiltonian, which in turn modifies the tangenttangent correlation function we expect [36]. Secondly, the channels in the cell have soft walls and are ductile. A "soft" confining geometry has been described before in references 101,102. In combination, the studies using bent channels and "soft" channel walls establish a promising background for the investigation of polymers in a cellular environment.

A comparison of our findings with literature data relies mostly on DNA experiments. Besides our experiments on vimentin filaments, experiments on confined actin filaments have been performed in the past by Köster et al.. Results from both studies have already been combined in our evaluation of Odijk's scaling law. Also, a number of similar experiments have been performed on DNA confined in nanochannels or nanoslits. A selection of papers on the topic are found in references $47,50,103$. Because of the short persistence length of the DNA strand of about $50 \mathrm{~nm}$, the confining channels are generally built with widths of as little as $30 \mathrm{~nm}$ and more or less rectangular cross-section [47]. The analysis of the fluorescently labeled DNA strands' conformation is based on a measurements of the end-to-end distance of the filaments, which have a well defined contour length. For DNA, a direct analysis of the contour is not possible due to the resolution limit of optical microscopy. Therefore, the deflection length featured in the scaling law is 
calculated directly from the end-to-end distances of the confined DNA strands. It is found that DNA strands comply with Odijk's scaling law for channel dimensions of up to $140 \mathrm{~nm}$.

In the case of DNA confined to nanochannels, there also is the possibility of an application beyond a polymer physics study. By employing very narrow channels, it is possible to observe fully stretched DNA 48,104. Fluorescent dyes which bind in a sequence dependent manner can be utilized to create a fluorescent DNA bar code, which can then be read from the fully stretched DNA [105].

\subsubsection{Reduction of configuration space}

As indicated by the comparatively large error associated with the measurement of the persistence length of freely fluctuating filaments (see chapter 6.3.3), statistics in the unconfined case are inferior to those in the confined case. This also becomes apparent when we consider the error associated with the tangent correlation functions plotted in figure 6.7, which increase strongly with decreasing confinement. Thus, the confinement of filaments and the reduction of configuration space this entails is very favorable from a statistical standpoint.

A filament covers a certain configuration space $C_{n}$, which is different for confined and free filaments. The configuration space of a filament of length $L$ confined between two plates with a distance $h$ between them is on the order of

$$
C_{n} \sim h L^{2}
$$

If we now consider a confining channel geometry with height $h$ and width $d$, we obtain a reduction of configuration space to

$$
C_{n} \sim h d L
$$

Also, the filament in a channel shows features which repeat after a certain distance, which in our case is the deflection length $\lambda[51$. This gives rise to a further reduction in configuration space to

$$
C_{n} \sim h \lambda d .
$$

The configuration space is reduced by a factor of $d / L \approx 10$ and at the same time a factor of $\lambda / L \approx 10$. In total, the configuration space is reduced by 2 orders of magnitude by employing a confining channel geometry. In this manner, the many repeat units $\lambda$ we observe in the microchannels greatly help us to improve the statistics of our measurements. 


\section{Chapter 7}

\section{Summary, conclusion and outlook}

In the previous chapters we have shown different studies conducted on fluorescently labeled vimentin intermediate filaments. Vimentin filaments are of great relevance in eukaryotic cells of mesenchymal origin, where their impairment plays a key role in different diseases. We have therefore performed studies on the assembly, disassembly and dynamic changes of the composition of the filaments. In part, these studies were performed by employing microfluidic devices. The use of microfluidic methods is also central for our experiments on confined vimentin filaments. Here, we can employ vimentin filaments as a model system for worm-like chains.

We have investigated the subunit exchange between vimentin intermediate filaments in vitro. Here, we have found that vimentin filaments do indeed exchange subunits. Notably, this process takes place without disrupting the filament's overall stability. We have also determined that the rate of the subunit exchange process is influenced by the filament's polymorphism. It is also very likely that the polymorphism of vimentin filaments makes the subunit exchange possible in the first place. Our results are also in good agreement with literature data gained from live cells, which means that the subunit exchange reaction is mostly independent of cellular regulatory mechanisms.

By employing microfluidic diffusion chambers, we aim to further our understanding of the assembly, disassembly and subunit exchange processes by observing vimentin filaments in situ and in real time. The characterization of the device with the help of simulations has been essential for the planning of our experiments. With experiments planned according to these simulations, we could observe the early assembly characteristics of fluorescently labeled vimentin in real time. Our results for the assembly characteristics compare favorably to bulk studies. In addition to the 
observation of the assembly characteristics, we gained first insights into the disassembly process of vimentin filaments by observing this process in diffusion chambers as well as in bulk samples. Our findings with regards to the disassembly reaction provide a foundation for further research in an area where literature data are largely missing. For both the assembly and disassembly experiments, the real time observation in our microfluidic setup is essential for our measurements. Thus, the concept of the microfluidic diffusion-chamber-device in general is promising for future experiments. However, the imaging conditions in the diffusion-chamber-device could be improved. Also, we found that interactions of vimentin with the chamber surfaces influence both the assembly and disassembly properties of the protein. We have determined that a lipid bilayer coating is a very good, but still imperfect, surface treatment that prevents surface interactions of vimentin to a large extent.

Future experiments employing the diffusion-chamber concept would depend on improvements to the coating system or methods for a faster exchange of solutions by means of fluid flow instead of diffusion. The ideal choice of surface coating for experiments on vimentin remains elusive. However, existing microfluidic techniques could be used to supply each diffusion chamber with a discrete in- and outlet controlled by a valve [106]. Thus, the exchange of solution in the chamber would require only a fraction of the time the diffusive process needs. However, there is a trade-off between the new possibilities this device design establishes and the conceptual difficulties of a more complex microfluidic device. In the end, the actual experiment would still rely on exchange of reagents by diffusion.

The diffusion-chamber-microfluidic-device could also be modified to be compatible with small angle X-ray scattering (SAXS) experiments. To this end we could use techniques developed by Brennich et al. and Weinhausen and Köster [13, 107]. The experiments that could be conducted on such a setup are in principle similar to those that we have performed in this thesis. A fluorescent dye would not be necessary to visualize the sample and instead X-rays would be used to analyze the sample. For instance, it would be possible to measure the changing X-ray signal during the assembly process of various intermediate filament types.

In the final part of our studies on vimentin filaments, we continue to employ microfluidic techniques to investigate the properties of vimentin filaments. We have studied the mechanical properties of vimentin filaments and have experimentally confirmed characteristics of worm-like chains which were expected from theoretical considerations. To this end, we have confined the filaments in micro channels and used surface effects in microfluidic devices to our advantage. The statistical ad- 
vantages we gain from observing confined filaments are apparent when we look at the reduction of configuration space of a filament in a microchannel by two orders of magnitude. This observation combined with the very large number of individual filament images we analyzed enables us to make strong conclusions on the properties of vimentin in particular and polymers described as worm-like chains in general. We have found that the persistence length of vimentin filaments is about $2 \mu \mathrm{m}$, which is in agreement with values previously found in other experiments. We were also able to show that the fluctuations of the confined filament in the imaging plane and perpendicular to this plane decouple. Furthermore, data gained from vimentin filaments together with data from actin filaments, which are both confined in the Odijk regime, allow us to completely confirm a scaling law formulated by Odijk, which connects the channel width and the persistence length of the filament to a characteristics length scale. Interestingly, we find that both actin and vimentin filaments can be described by the worm-like chain model despite strong differences in the molecular structure of both proteins.

Apart from the comprehensive relevance the interaction of vimentin filaments with the surfaces of the microfluidic devices has for our studies, a further property of the filaments is implied by our subunit exchange experiments as well as our consideration of vimentin filaments as a bundle of $\alpha$-helical coiled-coils. In the latter case, we find that the individual subunits in the filament form only loose bonds with their lateral neighbors. Exactly these loose bonds might be responsible for the easy release of subunits into the soluble pool, which is necessary for the exchange of subunits between vimentin filaments.

To expand on our experiments on confined vimentin filaments, it would be possible to employ wider channels. The reduced confinement of the filaments leads to different theoretical models that can be used to describe polymers [53,108. By again employing vimentin filaments as an example for an ideal worm-like chain, it would be possible to gain further insights into the different confinement regimes. A limiting factor in this regard is the finite length of the vimentin filament. We could thus only investigate confinement regimens with strong to moderate confinement. Regimes with very weak confinement are best investigated on filaments with a contour length of at least 100 times the persistence length, whereas for vimentin filaments only a factor of 50 is attainable.

This thesis was undertaken to further our understanding of the intermediate filament vimentin, with a focus on a possible subunit exchange mechanism and the filament's mechanical properties. We were able to show that a subunit exchange 
mechanism is present in vimentin filaments independently from cellular mechanisms. Experiments employing microfluidic techniques allowed us to further our understanding of the dynamic assembly and disassembly processes. Furthermore, we determined that vimentin filaments can be described as ideal worm-like chains. In combination these findings help us to understand the dynamic processes, which govern vimentin filaments in vitro and ultimately in vivo, in greater detail. 


\section{Appendix A}

\section{Vimentin synthesis}

In this chapter we describe the synthesis and purification process of vimentin protein expressed in E.coli bacteria. The plasmids used for the transfection of the E.coli were kindly provided by Harald Herrmann (DKFZ, Heidelberg, Germany). Mostly, protein was prepared by Susanne Bauch. Chemicals were obtained from Carl Roth (Karlsruhe, Germany), if no other supplier is listed.

\section{A.1 Expression in E.Coli}

$100 \mu \mathrm{L}$ E.Coli (T1 cells, \#T3017, Zymo research, Irvine, California, USA) are thawed on ice for 3 minutes. $1 \mu \mathrm{L}$ plasmid solution is added to the bacteria. Following an incubation time of 5 minutes, $50 \mu \mathrm{L}$ bacteria solution are spread on a lysogeny broth agar plate. The agar plate is incubated over night at $37^{\circ} \mathrm{C}$. Three individual bacteria colonies are collected from the agar plate and added to three separate reaction tubes containing $5 \mathrm{~mL}$ terrific broth solution (47.6 g terrific broth (SIGMA) and $8 \mathrm{ml} 99.5 \%$ glycerol per $1 \mathrm{~L}$ of solution). $5 \mu \mathrm{L}$ ampicillin solution (100 mg/ml ampicillin sodium salt, SIGMA) are added to the solution. The bacteria are incubated for $6 \mathrm{~h}$ at $37^{\circ} \mathrm{C}$ and $150 \mathrm{rpm}$ in an Ecotron incubator (Infors, Basel, Switzerland). After this incubation period each bacteria solution is transferred to $1 \mathrm{~L}$ of terrific broth containing $0.5 \mathrm{~mL}$ ampicillin and grown over night at $37^{\circ} \mathrm{C}$ and $150 \mathrm{rpm}$. Following this final bacteria growth step, bacteria are separated from the medium by centrifugation at $4500 \mathrm{~g}$ for 12 minutes at $4^{\circ} \mathrm{C}$. The resulting pellet can be stored at $-20^{\circ} \mathrm{C}$ before the purification process. 


\section{A.2 Purification of vimentin from bacteria pellet}

\section{A.2.1 Buffers}

Buffer 150 mM TRIS, 25\% saccharose, 1 mM EDTA; adjusted to pH 8.0 with $\mathrm{HCl}$ Buffer $2120 \mathrm{mg}$ lysozyme in $12 \mathrm{~mL}$ of buffer $\mathbf{1}$

Buffer 320 mM TRIS, 2mM EDTA, 200 mM NaCL, 1\% NP40, 1\% DOC; adjusted to $\mathrm{pH} 7.5$ with $\mathrm{HCl}$

Buffer 410 mM TRIS, 0.5\% TritonX-100, 5 mM EDTA; adjusted to pH 8.0 with $\mathrm{HCl}$

Buffer $51.5 \mathrm{M} \mathrm{KCl}$ in buffer 4

Buffer 610 mM TRIS, 0.1 mM EDTA; adjusted to pH 8.0 with HCL

Buffer $750 \mathrm{mg} / \mathrm{mL}$ DNAse1 (Sigma-Aldrich, St. Louis, USA) in $100 \mathrm{mM}$ TRIS, $100 \mathrm{mM} \mathrm{NaCl}$; adjusted to $\mathrm{pH} 7.5$ with $\mathrm{HCl}$

Buffer 810 mg/mL RNaseA (Roche Diagnostics, Mannheim, Germany), in $100 \mathrm{mM}$ TRIS; adjusted to $\mathrm{pH} 7.5$ with $\mathrm{HCl}$

Buffer 9 PMSF in ethanol, saturated solution

Buffer 1050 mM TRIS, $10 \mathrm{mM}$ EDTA, $1 \mathrm{mM}$ EGTA, $1 \mathrm{mM}$ DTT; adjusted to $\mathrm{pH} 8.4$ with $\mathrm{HCl}$

Buffer $11400 \mathrm{~mL} 10 \mathrm{M}$ urea, $50 \mathrm{~mL}$ of buffer 9, $0.5 \mathrm{~mL}$ DTT; adjusted to $\mathrm{pH} 7.5$ with $\mathrm{HCl}$

Buffer 1230 mL DEAE (diethylaminoethyl) sepharose solution (GE Healthcare, Munich, Germany), $30 \mathrm{~mL}$ of buffer $\mathbf{1 0}$

Buffer $1330 \mathrm{~mL}$ CM (carboxymethyl) sepharose solution (GE Healthcare), $30 \mathrm{~mL}$ of buffer 10

\section{A.2.2 Centrifugation}

1. Bacteria pellets are dissolved in $48 \mathrm{~mL}$ of buffer 1 . The pellet is homogenized in two dounce homogenisators (VWR, Darmstadt, Germany, $20 \mathrm{~mm}$ pestle) under the addition of $6 \mathrm{~mL}$ of buffer 2 . The homogenisators are kept on ice during this process.

2. $0.3 \mathrm{~mL} 1 \mathrm{M} \mathrm{MgCl}_{2}, 30 \mu \mathrm{L}$ buffer $6,33 \mu \mathrm{L}$ buffer $7,0.3 \mathrm{~mL}$ buffer 8 and $0.6 \mathrm{~mL}$ NP40 are added to each homogenisator followed by further homogenization and incubation on ice for 10 minutes. 
3. $60 \mathrm{~mL}$ buffer 3, $0.6 \mathrm{~mL} 1 \mathrm{M} \mathrm{DTT,} 0.9 \mathrm{~mL}$ buffer 8 and $0.3 \mathrm{~mL} 50 \mathrm{mM}$ pefabloc are added before a final homogenization.

4. Solutions from both homogenisators is centrifuged for 25 minutes at $10000 \mathrm{~g}$ at $4^{\circ} \mathrm{C}$ (Beckmann centrifuge J26XP, rotor Ja30.50TI, Beckmann Coulter, Krefeld, Germany).

5. Supernatant is discarded. Pellets are dissolved in $120 \mathrm{~mL}$ of buffer 4, $0.12 \mathrm{~mL}$ $1 \mathrm{M}$ DTT, $0.6 \mathrm{~mL}$ of buffer 8 and $0.3 \mathrm{~mL} 50 \mathrm{mM}$ pefabloc by homogenization followed by 10 minutes incubation on ice.

6. Centrifugation for 20 minutes at $10000 \mathrm{~g}$ and $4^{\circ} \mathrm{C}$.

7. Supernatant is discarded. Pellets are dissolved in $120 \mathrm{~mL}$ of buffer 4a, $0.12 \mathrm{~mL} 1 \mathrm{M}$ DTT, $0.6 \mathrm{~mL}$ of buffer 8 and $0.3 \mathrm{~mL} 50 \mathrm{mM}$ pefabloc by homogenization followed by 10 minutes incubation on ice.

8. Centrifugation for 20 minutes at $10000 \mathrm{~g}$ and $4^{\circ} \mathrm{C}$.

9. Supernatant is discarded. Pellets are dissolved in $120 \mathrm{~mL}$ of buffer 4, $0.12 \mathrm{~mL}$ $1 \mathrm{M}$ DTT, $0.6 \mathrm{~mL}$ of buffer 8 and $0.3 \mathrm{~mL} 50 \mathrm{mM}$ pefabloc by homogenization followed by 10 minutes incubation on ice.

10. Centrifugation for 20 minutes at $10000 \mathrm{~g}$ and $4^{\circ} \mathrm{C}$.

11. Supernatant is discarded. Pellets are dissolved in $80 \mathrm{~mL}$ buffer 5, $80 \mu \mathrm{L} 1 \mathrm{M}$ DTT, $0.4 \mathrm{~mL}$ of buffer 8 and $0.2 \mathrm{~mL} 50 \mathrm{mM}$ pefabloc by homogenization followed by 15 minutes. incubation on ice.

12. Centrifugation for 10 minutes at $10000 \mathrm{~g}$ and $4^{\circ} \mathrm{C}$.

13. The pellet is dissolved in $28.5 \mathrm{~mL} 9.5 \mathrm{M}$ urea, $0.3 \mathrm{~mL} 1 \mathrm{M}$ TRIS-HCl (pH 7.5), $0.3 \mathrm{~mL} 0.5 \mathrm{M}$ EDTA, $0.3 \mathrm{~mL}$ of buffer 8 by homogenization.

14. Centrifugation for 60 minutes at $100000 \mathrm{~g}$ and $20^{\circ} \mathrm{C}$ (Beckmann-UZ L90K, rotor TI70)

15. Supernate is kept, $10 \mu \mathrm{L} \mathrm{MAC} \mathrm{solution} \mathrm{is} \mathrm{added} \mathrm{per} \mathrm{mL}$ of supernate. Storage at $-20^{\circ} \mathrm{C}$. 


\section{A.2.3 Ion exchange chromatography}

\section{Anion exchange}

An ion gradient mixer $(2 \times 50 \mathrm{~mL}$, Schütt 24, Göttingen, Germany) is filled with $40 \mathrm{~mL}$ of buffer 11 in the chamber near the outlet and $40 \mathrm{~mL}$ of buffer 11 with $0.448 \mathrm{~g} \mathrm{KCl}$ in the rear chamber. The column (25 mm diameter, $200 \mathrm{~mm}$ long) is filled with buffer 12 and washed with $40 \mathrm{~mL}$ of buffer 11. The protein solution is added on the column. A slow flow passes through the column, maintaining a constant filling level. The eluate of the column is collected. $40 \mathrm{~mL}$ of buffer $\mathbf{1 1}$ are slowly added to the column. After the collected volume reaches the dead volume of the column, sample collection begins. The gradient mixer is connected to the column and flow begins at a rate of about 4 drops per second. The column volume is kept constant by the regulation of inflow and outflow. The eluate is collected in sample volumes of $1.5 \mathrm{~mL}$. Protein content is monitored using a NanoDrop (see chapter 3.1.2). The Samples containing protein are combined before the next step.

\section{Cation exchange}

The process for the kation exchange is very similar to the anion exchange. The column material is exchanged. The colummn is filled with buffer 13 instead of buffer 12 and $0.896 \mathrm{~g} \mathrm{KCl}$ are added in the rear chamber.

After the cation exchange, $10 \mu \mathrm{L} 1 \mathrm{M}$ MAC are added per $\mathrm{mL}$ of protein solution. The protein is stored at $-80^{\circ} \mathrm{C}$. 


\section{Appendix B}

\section{Abbreviations}

$a$

$A_{c h}$

$A_{F}$

$A_{\text {sub }}$

$A_{r t}$

AFM

ATP

$B_{\text {sub }}$

BSA

$\mathrm{C}$

CCD

$\mathrm{CHCl}_{3}$

CMOS

$C_{n}$

d

$D_{K C l}$

$D_{t}$

$D_{t, 37^{\circ} \mathrm{C}}$

$D_{t}^{\|}$

$D_{t}^{\perp}$

$D_{t, \exp }$ scaling parameter

surface-area of a diffusion-chamber

average cross-section area a filament occupies

percentage of exchanged subunits

surface-area of a reaction tube

atomic force microscopy

adenosine triphosphate

exchanged subunits with background as reference

bovine serum albumin

concentration

charge-coupled device

chloroform

complementary metal-oxide-semiconductor

configuration space of a filament

channel width

diffusion coefficient for potassium chloride

isotropic diffusion coefficient for tetrameric vimentin

isotropic diffusion coefficient for tetrameric vimentin at $37^{\circ} \mathrm{C}$

anisotropic diffusion coefficient for tetrameric vimentin paral-

lel to the long axis

anisotropic diffusion coefficient for tetrameric vimentin perpendicular to the long axis

experimentally measured diffusion coefficient for tetrameric vimentin 
$D_{U L F} \quad$ isotropic diffusion coefficient for unit length filament vimentin

DHPC 1,2-diheptanoyl-sn-glycero-3-phosphocholine

DOPC 1,2-dioleoyl-sn-glycero-3-phosphocholine

DMSO dimethyl sulfoxide

DNA desoxyribonucleic acid

DTT dithiothreitol

EDTA ethylenediaminetetraacetic acid

EGTA ethylene glycol tetraacetic acid

FRET Förster resonance energy transfer or

fluorescence resonance energy transfer

FRAP fluorescence recovery after photobleaching

GFA glial fibrillary acidic protein

GFP green fluorescent protein

green vimentin with AlexaFlour 488 fluorescent label

$G^{*} \quad$ linear viscoelastic shear modolus

HOPG highly oriented pyrolytic graphite

$h \quad$ channel height

IF intermediate filament

$I_{b a c k} \quad$ background fluorescence intensity

$I_{e x} \quad$ exchanged subunit fluorescence intensity

$I_{f i l} \quad$ fluorescence intensity of a red filament

$K \quad$ potential strength

$k \quad$ spring constant

$k_{B} \quad$ Boltzmann constant

$L \quad$ contour length

$L_{0} \quad$ characteristic length scale

$L_{P} \quad$ persistence length

$L_{P, a p p} \quad$ apparent persistence length

$L_{P, c c} \quad$ persistence length of a $\alpha$-helical coiled-coil

$L_{P, \text { dimer }} \quad$ persistence length of dimeric vimentin

$L_{P, \text { rhe }} \quad$ persistence length measured with rheology methods

$L_{P, \text { bundle }} \quad$ persistence length of a bundle of identical filaments

$L_{P, \text { filament }} \quad$ persistence length of an individual filament

$L_{x} \quad$ projected contour length

$l \quad$ correlation distance 
$M_{L} \quad$ mass per length

MAC methylaminhydrochlorid

mCherry fluorescent protein with red emmision spectrum

$N \quad$ number of individual filaments in a bundle

N.A. numerical aperture

$N_{k} \quad$ number of links in a polymer chain

$p \quad$ ratio of filament length and diameter

$p_{0} \quad$ fraction of protein without label

$R \quad$ end-to-end distance

Re Reynolds number

red vimentin with ATTO 647N fluorescent label

SUV Small Unilaminar Vesicle

T temperature

$t \quad$ tangent vector

Pe Peclet number

TRIS tris(hydroxymethyl)aminomethane

PDMS poly(dimethylsiloxane)

PLL-g-PEG poly(L-lysine)-g-poly(ethylene glycol)

PMSF phenylmethanesulfonylfluoride

$V_{c d} \quad$ volume of a diffusion-chamber

$V_{r t} \quad$ volume of a reation tube

WLC worm-like chain

$U_{0}$

$\mathrm{ULF}$

characteristic velocity scale

unit length filament

cross-linking exponent for bundles

cross-linking exponent for intermediate filaments

extinction coefficient for vimentin

$\eta_{0} \quad$ viscosity of water at $20^{\circ} \mathrm{C}$

$\theta \quad$ correlation angle

$\kappa \quad$ bending stiffness

$\lambda \quad$ deflection length

$\nu \quad$ form factor in rod diffusion

$\rho \quad$ fluid density

$\tau_{1 / 2} \quad$ half life time 


\section{Bibliography}

[1] Shabbir SH, Cleland MM, Goldman RD, Mrksich M. Geometric control of vimentin intermediate filaments. Biomaterials. 2014;35(5):1359-1366.

[2] Li R, Johnson AB, Salomons G, Goldman JE, Naidu S, Quinlan R, Cree B, Ruyle SZ, Banwell B, D'Hooghe M, et al. Glial fibrillary acidic protein mutations in infantile, juvenile, and adult forms of Alexander disease. Annals of neurology. 2005;57(3):310-326.

[3] Bolling M, Lemmink H, Jansen G, Jonkman M. Mutations in KRT5 and KRT14 cause epidermolysis bullosa simplex in $75 \%$ of the patients. British Journal of Dermatology. 2011;164(3):637-644.

[4] Müller M, Bhattacharya SS, Moore T, Prescott Q, Wedig T, Herrmann H, Magin TM. Dominant cataract formation in association with a vimentin assembly disrupting mutation. Human molecular genetics. 2009;18(6):1052-1057.

[5] Strelkov S, Herrmann H, Aebi U. Molecural Architecture of Intermediate Filaments. BioEssasys. 2003;25(3):243-251.

[6] Szeverenyi I, Cassidy AJ, Chung CW, Lee BT, Common JE, Ogg SC, Chen H, Sim SY, Goh WL, Ng KW, et al. The Human Intermediate Filament Database: comprehensive information on a gene family involved in many human diseases. Human mutation. 2008;29(3):351-360.

[7] Herrmann H, Aebi U. Intermediate filaments: Molecular structure, assembly mechanism, and integration into functionally distinct intracellular scaffolds. Annual Review of Biochemistry. 2004;73:749-789.

[8] Zwerger M, Medalia O. From lamins to lamina: a structural perspective. Histochemistry and cell biology. 2013;pp. 1-10. 
[9] Herrmann H, Häner M, Brettel M, Ku NO, Aebi U. Characterization of distinct early assembly units of different intermediate filament proteins. Journal of Molecular Biology. 1999;286(5):1403 - 1420.

[10] Portet S, Mücke N, Kirmse R, Langowski J, Beil M, Herrmann H. Vimentin Intermediate Filament Formation: In vitro Measurement and Mathematical Modeling of the Filament Length Distribution During Assembly. Langmuir. 2009;25(15):8817-23.

[11] Herrmann H, Aebi U. Intermediate Filament Assembly: Fibrillogenesis is Driven by Decisive Dimer-Dimer Interactions. Current Opinion in Structural Biology. 1998;8(2):177 - 185.

[12] Herrmann H, Häner M, Brettel M, Müller SA, Goldie KN, Fedtke B, Lustig A, Franke WW, Aebi U. Structure and assembly properties of the intermediate filament protein vimentin: the role of its head, rod and tail domains. Journal of molecular biology. 1996;264(5):933-953.

[13] Brennich ME, Nolting JF, Dammann C, Nöding B, Bauch S, Herrmann H, Pfohl T, Köster S. Dynamics of intermediate filament assembly followed in micro-flow by small angle X-ray scattering. Lab on a Chip. 2011;11(4):708-716.

[14] Ackbarow T, Buehler MJ. Molecular mechanics of stutter defects in vimentin intermediate filaments. Experimental mechanics. 2009;49(1):79-89.

[15] Kirmse R, Portet S, Mücke N, Aebi U, Herrmann H, Langowski J. A Quantitative Kinetic Model for the in Vitro Assembly of Intermediate Filaments from Tetrameric Vimentin. Journal of Biological Chemistry. 2007;282(25):1856318572.

[16] Winheim S, Hieb AR, Silbermann M, Surmann EM, Wedig T, Herrmann H, Langowski J, Mücke N. Deconstructing the late phase of vimentin assembly by total internal reflection fluorescence microscopy (TIRFM). PloS one. 2011; 6(4):e19202.

[17] Çolakoğlu G, Brown A. Intermediate filaments exchange subunits along their length and elongate by end-to-end annealing. The Journal of cell biology. 2009; 185(5):769-777. 
[18] Meier M, Padilla GP, Herrmann H, Wedig T, Hergt M, Patel TR, Stetefeld J, Aebi U, Burkhard P. Vimentin coil 1A-a molecular switch involved in the initiation of filament elongation. Journal of molecular biology. 2009;390(2):245261.

[19] Chernyatina AA, Nicolet S, Aebi U, Herrmann H, Strelkov SV. Atomic structure of the vimentin central $\alpha$-helical domain and its implications for intermediate filament assembly. Proceedings of the National Academy of Sciences. 2012;109(34):13620-13625.

[20] Ngai J, Coleman TR, Lazarides E. Localization of newly synthesized vimentin subunits reveals a novel mechanism of intermediate filament assembly. Cell. 1990;60(3):415-427.

[21] Vikstrom KL, Lim SS, Goldman RD, Borisy GG. Steady state dynamics of intermediate filament networks. The Journal of cell biology. 1992;118(1):121129.

[22] Uchida A, Çolakoğlu G, Wang L, Monsma PC, Brown A. Severing and endto-end annealing of neurofilaments in neurons. Proceedings of the National Academy of Sciences. 2013;110(29):E2696-E2705.

[23] Higashi N, Kojima N, Miura M, Imai K, Sato M, Senoo H. Cell-cell junctions between mammalian (human and rat) hepatic stellate cells. Cell and tissue research. 2004;317(1):35-43.

[24] Guo M, Ehrlicher AJ, Mahammad S, Fabich H, Jensen MH, Moore JR, Fredberg JJ, Goldman RD, Weitz DA. The Role of Vimentin Intermediate Filaments in Cortical and Cytoplasmic Mechanics. Biophysical journal. 2013; 105(7):1562-1568.

[25] Seltmann K, Fritsch AW, Käs JA, Magin TM. Keratins significantly contribute to cell stiffness and impact invasive behavior. Proceedings of the National Academy of Sciences. 2013;110(46):18507-18512.

[26] Herrmann H, Strelkov SV, Burkhard P, Aebi U. Intermediate filaments: primary determinants of cell architecture and plasticity. The Journal of clinical investigation. 2009;119(7):1772. 
[27] Mendez MG, Kojima SI, Goldman RD. Vimentin induces changes in cell shape, motility, and adhesion during the epithelial to mesenchymal transition. The Federation of American Societies for Experimental Biology Journal. 2010; 24(6):1838-1851.

[28] Iwatsuki H, Suda M. Seven kinds of intermediate filament networks in the cytoplasm of polarized cells: structure and function. Acta histochemica et cytochemica. 2010;43(2):19.

[29] Qin Z, Kreplak L, Buehler MJ. Hierarchical structure controls nanomechanical properties of vimentin intermediate filaments. PLoS One. 2009;4(10):e7294.

[30] Kreplak L, Herrmann H, Aebi U. Tensile properties of single desmin intermediate filaments. Biophysical journal. 2008;94(7):2790-2799.

[31] Kreplak L, Bär H, Leterrier J, Herrmann H, Aebi U. Exploring the mechanical behavior of single intermediate filaments. Journal of molecular biology. 2005; 354(3):569-577.

[32] Schopferer M, Bär H, Hochstein B, Sharma S, Mücke N, Herrmann H, Willenbacher N. Desmin and Vimentin Intermediate Filament Networks: Their Viscoelastic Properties Investigated by Mechanical Rheometry. Journal of Molecular Biology. 2009;388(1):133 - 143.

[33] Assessing the Flexibility of Intermediate Filaments by Atomic Force Microscopy. Journal of Molecular Biology. 2004;335(5):1241 - 1250.

[34] Lichtenstern T, Mücke N, Aebi U, Mauermann M, Herrmann H. Complex formation and kinetics of filament assembly exhibited by the simple epithelial keratins K8 and K18. Journal of structural biology. 2012;177(1):54-62.

[35] Howard J. Mechanics of motor proteins and the cytoskeleton. Sinauer Associates Sunderland, MA. 2001.

[36] Köster S, Kierfeld J, Pfohl T. Characterization of Single Semiflexible Filaments under Geometric Constraints. European Physical Journal E. 2008;25(4):439449.

[37] Gittes F, Mickey B, Nettleton J, Howard J. Flexural Rigidity of Microtubules and Actin Filaments Measured from Thermal Fluctuations in Shape. The Journal of Cell Biology. 1993;120(4):923-934. 
[38] Ott A, Magnasco M, Simon A, Libchaber A. Measurement of the persistence length of polymerized actin using fluorescence microscopy. Physical Review E. 1993;48(3):R1642-R1645.

[39] Adami R, Choquet D, Grazi E. Rhodamine phalloidin F-actin. European journal of biochemistry. 1999;263(1):270-275.

[40] Liu X, Pollack GH. Mechanics of F-actin characterized with microfabricated cantilevers. Biophysical journal. 2002;83(5):2705-2715.

[41] Stone HA, Stroock AD, Ajdari A. Engineering flows in small devices: microfluidics toward a lab-on-a-chip. The Annual Review of Fluid Mechanics. 2004;36:381-411.

[42] Squires TM, Quake SR. Microfluidics: Fluid physics at the nanoliter scale. Reviews of modern physics. 2005;77(3):977.

[43] Nge PN, Rogers CI, Woolley AT. Advances in microfluidic materials, functions, integration, and applications. Chemical reviews. 2013;113(4):2550-2583.

[44] Wong I, Ho CM. Surface molecular property modifications for poly (dimethylsiloxane)(PDMS) based microfluidic devices. Microfluidics and nanofluidics. 2009;7(3):291-306.

[45] Makamba H, Kim JH, Lim K, Park N, Hahn JH. Surface modification of poly (dimethylsiloxane) microchannels. Electrophoresis. 2003;24(21):3607-3619.

[46] Deshpande S, Pfohl T. Hierarchical self-assembly of actin in microconfinements using microfluidics. Biomicrofluidics. 2012;6:034120.

[47] Reisner W, Morton K, Riehn R, Wang Y, Yu Z, Rosen M, Sturm J, Chou S, Frey E, Austin R. Statics and Dynamics of Single DNA Molecules Confined in Nanochannels. Physical Review Letters. 2005;94(19):196101.

[48] Kim Y, Kim KS, Kounovsky KL, Chang R, Jung GY, Jo K, Schwartz DC, et al. Nanochannel confinement: DNA stretch approaching full contour length. Lab on a Chip. 2011;11(10):1721-1729.

[49] Su T, Das SK, Xiao M, Purohit PK. Transition between two regimes describing internal fluctuation of DNA in a nanochannel. PLoS One. 2011;6(3):e16890. 
[50] Bonthuis DJ, Meyer C, Stein D, Dekker C. Conformation and dynamics of DNA confined in slitlike nanofluidic channels. Physical Review Letters. 2008; 101(10):108303.

[51] Odijk T. The Statistics and Dynamics of Confined or Entangled Stiff Polymers. Macromolecules;(8):1340-1344.

[52] de Gennes PG. Scaling Concepts in Polymer Physics. Cornell University Press. 1979.

[53] Odijk T. Scaling theory of DNA confined in nanochannels and nanoslits. Physical Review E. 2008;77(6):060901.

[54] Kooijman M, Bloemendal M, Traub P, van Grondelle R, van Amerongen H. Transient electric birefringence study of intermediate filament formation from vimentin and glial fibrillary acidic protein. Journal of Biological Chemistry. 1997;272(36):22548-22555.

[55] Life Technologies Corporation. AlexaFluor 488 spectra http: //www.lifetechnologies.com/de/de/home/life-science/cell-analysis/ labeling-chemistry/fluorescence-spectraviewer.html. Accessed on 27.01.2014;

[56] Life Technologies Corporation. Fluorescent dyes quantum yield http://www.lifetechnologies.com/de/de/ home/references/molecular-probes-the-handbook/tables/ fluorescence-quantum-yields-and-lifetimes-for-alexa-fluor-dyes.html. Accessed on 27.01.2014;

[57] ATTO-TEC GmbH. Fluorescent dyes quantum yield https: //wWw.atto-tec.com/attotecshop/product_info.php?info=p114_atto-647n. html\&XTCsid=cb9bdb08ac6ecc5326376b238ffb7982. Accessed on 27.01.2014;

[58] Dyomics GmbH. Dyomics DY-521XL datasheet https://www.atto-tec. com/attotecshop/product_info.php?info=p114_atto-647n.html\&XTCsid= cb9bdb08ac6ecc5326376b238ffb7982. Accessed on 27.01.2014;.

[59] Life Technologies Corporation. AlexaFluor Thiol-Reactive Probes manual http://tools.lifetechnologies.com/content/sfs/manuals/mp00003.pdf. Accessed on 27.01.2014;. 
[60] Quake S, Scherer A. From Micro- to Nanofabrication with Soft Materials. Science. 2000;290:1536-1540.

[61] Duffy D, McDonald J, Schueller O, Whitesides G. Rapid Prototyping of Microfluidic Systems in Poly(dimethylsiloxane). Analytical Chemistry. 1998; 70(23):4974-4984.

[62] Delamarche E, Bernard A, Schmid H, Bietsch A, Michel B, Biebuyck H. Microfluidic Networks for Chemical Patterning of Substrates: Design and Application to Bioessays. Journal of the American Chemical Society. 1998; $120(3): 500-508$.

[63] Xia Y, Whitesides G. Soft Lithography. Angewandte Chemie International Edition. 1998;37:550-575.

[64] MicroChem Corp. SU8 datasheet microchem.com/pdf/ SU-82000DataSheet2000_5thru2015Ver4.pdf. Accessed on 27.01.2014;

[65] Watson J, Baron M. The Behaviour of Water in Poly(dimethylsiloxane). Journal of Membrane Science. 1996;110:47-57.

[66] Verneuil E, Buguin A, Silberzan P. Permeation-Induced Flows: Consequences for Silicone-Based Microfluidics. Europhysics Letters. 2004;63(3):412-418.

[67] Nakanishi K, Sakiyama T, Imamura K. On the adsorption of proteins on solid surfaces, a common but very complicated phenomenon. Journal of Bioscience and Bioengineering. 2001;91(3):233-244.

[68] Banerjee I, Pangule RC, Kane RS. Antifouling coatings: recent developments in the design of surfaces that prevent fouling by proteins, bacteria, and marine organisms. Advanced Materials. 2011;23(6):690-718.

[69] Marie R, Beech JP, Vörös J, Tegenfeldt JO, Höök F. Use of PLL-g-PEG in micro-fluidic devices for localizing selective and specific protein binding. Langmuir. 2006;22(24):10103-10108.

[70] Yang T, Jung Sy, Mao H, Cremer PS. Fabrication of phospholipid bilayercoated microchannels for on-chip immunoassays. Analytical chemistry. 2001; 73(2):165-169. 
[71] Persson F, Fritzsche J, Mir KU, Modesti M, Westerlund F, Tegenfeldt JO. Lipid-based passivation in nanofluidics. Nano letters. 2012;12(5):2260-2265.

[72] Olympus Corporation of the Americas. Filter cube wavelengths olympusamerica.com/seg_section/uis2/seg_uis2_mr_umniba3.asp. Accessed on 27.01.2014;

[73] Förster T. Zwischenmolekulare Energiewanderung und Fluoreszenz. Annalen der Physik. 1948;437(1-2):55-75.

[74] Hillisch A, Lorenz M, Diekmann S. Recent advances in FRET: distance determination in protein-DNA complexes. Current opinion in structural biology. 2001;11(2):201-207.

[75] Herrmann H, Kreplak L, Aebi U. Isolation, Characterization, and In Vitro Assembly of Intermediate Filaments. Methods in cell biology. 2004;78:3-24.

[76] Korson L, Drost-Hansen W, Millero FJ. Viscosity of water at various temperatures. The Journal of Physical Chemistry. 1969;73(1):34-39.

[77] Eriksson J, Dechat T, Grin B, Helfand B, Mendez M, Pallari HM, Goldman R. Introducing Intermediate Filaments: from Discovery to Disease. The Journal of Clinical Investigation. 2009;119(7):1763-1771.

[78] Sejersen T, Lendahl U. Transient expression of the intermediate filament nestin during skeletal muscle development. Journal of Cell Science. 1993; 106(4):1291-1300.

[79] Michalczyk K, Ziman M. Nestin structure and predicted function in cellular cytoskeletal organisation. Histology and Histopathology. 2005;(20):665-671.

[80] Bornemann A, Schmalbruch H. Desmin and vimentin in regenerating muscles. Muscle \& nerve. 1992;15(1):14-20.

[81] Steinert PM, Chou YH, Prahlad V, Parry DA, Marekov LN, Wu KC, Jang SI, Goldman RD. A high molecular weight intermediate filament-associated protein in bhk-21 cells is nestin, a type VI intermediate filament protein limited co-assembly in vitro to form heteropolymers with type III vimentin and type IV $\alpha$-internexin. Journal of Biological Chemistry. 1999;274(14):9881-9890. 
[82] Chou YH, Khuon S, Herrmann H, Goldman RD. Nestin promotes the phosphorylation-dependent disassembly of vimentin intermediate filaments during mitosis. Molecular biology of the cell. 2003;14(4):1468-1478.

[83] Wickert U, Mücke N, Wedig T, Müller SA, Aebi U, Herrmann H. Characterization of the in vitro co-assembly process of the intermediate filament proteins vimentin and desmin: mixed polymers at all stages of assembly. European journal of cell biology. 2005;84(2):379-391.

[84] Harned HS, Nuttall RL. The diffusion coefficient of potassium chloride in dilute aqueous solution. Journal of the American Chemical Society. 1947; 69(4):736-740.

[85] Tirado MM, Martinez CL, de la Torre JG. Comparison of theories for the translational and rotational diffusion coefficients of rod-like macromolecules. Application to short DNA fragments. The Journal of chemical physics. 1984; 81:2047.

[86] Sokolova AV, Kreplak L, Wedig T, Mücke N, Svergun DI, Herrmann H, Aebi U, Strelkov SV. Monitoring intermediate filament assembly by small-angle x-ray scattering reveals the molecular architecture of assembly intermediates. Proceedings of the National Academy of Sciences. 2006;103(44):16206-16211.

[87] Brennich ME, Bauch S, Vainio U, Wedig T, Herrmann H, Köster S. Impact of ion valency on the assembly of vimentin studied by quantitative small angle X-ray scattering. Soft Matter. 2014;10:2059-2068.

[88] Dammann C, Nöding B, Köster S. Vimentin networks at tunable ionconcentration in microfluidic drops. Biomicrofluidics. 2012;6:022009.

[89] Nöding B, Köster S. Intermediate Filaments in Small Configuration Spaces. Physical Review Letters. 2012;108(8):088101.

[90] Kratky O, Porod G. Röntgenuntersuchung gelöster Fadenmoleküle. Recueil des Travaux Chimiques des Pays-Bas. 1949;68(12):1106-1122.

[91] Köster S, Steinhauser D, Pfohl T. Brownian Motion of Actin Filaments in Confining Microchannels. Journal of Physics: Condensed Matter. 2005; 17(49):4091-4104. 
[92] Köster S. Biological Matter in Microfluidic Environment - from Single Molecules to Self-Assembly. Dissertation, University of Göttingen. 2006.

[93] Kierfeld J, Stark H. Private Communications;.

[94] Li H, Shen T, Smith M, Fujiwara I, Vavylonis D, Huang X. Automated Actin Filament Segmentation, Tracking and Tip Elongation Measurements Based on Open Active Contour Models. Piscataway, NJ, USA: IEEE Press. 2009.

[95] Kierfeld J, Lipowsky R. Unbundling and Desorption of Semiflexible Polymers. Europhysics Letters. 2003;62(2):285-291.

[96] Nöding B. Dynamic Measurements of Individual Intermediate Filaments. Diploma Thesis, University of Göttingen. 2010.

[97] Sousa D, Cammarato A, Jang K, Graceffa P, Tobacman LS, Li XE, Lehman W. Electron microscopy and persistence length analysis of semi-rigid smooth muscle tropomyosin strands. Biophysical journal. 2010;99(3):862-868.

[98] Hvidt S, Ferry JD, Roelke DL, Greaser ML. Flexibility of light meromyosin and other coiled-coil $\alpha$-helical proteins. Macromolecules. 1983;16(5):740-745.

[99] Wolgemuth CW, Sun SX. Elasticity of $\alpha$-helical coiled coils. Physical Review Letters. 2006;97(24):248101.

[100] Heussinger C, Bathe M, Frey E. Statistical Mechanics of Semiflexible Bundles of Wormlike Polymer Chains. Physical Review Letters. 2007;99(4):048101.

[101] Brochard-Wyart F, Tanaka T, Borghi N, de Gennes PG. Semiflexible polymers confined in soft tubes. Langmuir. 2005;21(9):4144-4148.

[102] Avramova K, Milchev A. Polymer chains in a soft nanotube: A Monte Carlo Study. The Journal of chemical physics. 2006;124(2):024909-024909.

[103] Huang A, Bhattacharya A. DNA confined in a two-dimensional strip geometry. arXiv preprint arXiv:13121298. 2013;

[104] Persson F, Tegenfeldt JO. DNA in nanochannels-directly visualizing genomic information. Chemical Society Reviews. 2010;39(3):985-999. 
[105] Jo K, Dhingra DM, Odijk T, de Pablo JJ, Graham MD, Runnheim R, Forrest D, Schwartz DC. A single-molecule barcoding system using nanoslits for DNA analysis. Proceedings of the National Academy of Sciences. 2007;104(8):26732678.

[106] Unger MA, Chou HP, Thorsen T, Scherer A, Quake SR. Monolithic microfabricated valves and pumps by multilayer soft lithography. Science. 2000; 288(5463):113-116.

[107] Weinhausen B, Köster S. Microfluidic devices for X-ray studies on hydrated cells. Lab on a Chip. 2013;13(2):212-215.

[108] Reisner W, Pedersen JN, Austin RH. DNA confinement in nanochannels: physics and biological applications. Reports on Progress in Physics. 2012; 75(10):106601. 


\section{List of publications}

- Bernd Nöding and Sarah Köster:

Intermediate Filaments in Small Configuration Spaces, Physical Review Letters 108 (2012), 088101.

- Christian Dammann, Bernd Nöding and Sarah Köster:

Vimentin Networks at Tunable Ion-Concentration in Microfluidic Drops, Biomicrofluidics 6 (2012), 022009.

- Martha E. Brennich, Jens-F. Nolting, Christian Dammann, Bernd Nöding, Susanne Bauch, Harald Herrmann, Thomas Pfohl and Sarah Köster:

Dynamics of intermediate filament assembly followed in micro-flow by small angle x-ray scattering, Lab on a Chip 11 (2011), 708-716. 


\section{Danksagung}

An dieser Stelle möchte ich mich bei allen Personen bedanken, die dazu beigetragen haben, dass diese Arbeit entstehen konnte.

Sarah Köster möchte ich dafür danken, dass sie mir die Gelegenheit gab diese Dissertation in ihrer Arbeitsgruppe anzufertigen. Ich bin für die umfangreiche Betreuung und die vielen Gelegenheiten andere Arbeitsgruppen und Konferenzen zu besuchen dankbar.

Detlev Schild danke ich für sein Engagement in meinem Betreuungsausschuss, sowie für das Koreferat dieser Dissertation. Für die freundliche Einführung in den Umgang mit einem FCS Aufbau in seiner Arbeitsgruppe bin ich ihm sehr dankbar.

Thomas Burg danke ich für sein Engagement in meinem Betreuungsausschuss, sowie für die Teilnahme als Prüfer bei der Verteidigung dieser Dissertation.

Helmut Grubmüller, Andreas Janshoff und Florian Rehfeldt möchte ich dafür danken, dass sie sich als Prüfer bei der Verteidigung dieser Dissertation angeboten haben.

Harald Herrmann hat uns freundlicherweise das Plasmid zur Herstellung von Vimentin zur Verfügung gestellt, ohne das unsere Experimente so nicht möglich gewesen wären. Bei vielen Gelegenheiten haben wir über die Eigenschaften von Vimentin gesprochen, hierbei habe ich viele interessante und wichtig Details gelernt.

Norbert Mücke hat in angeregten Diskussionen über die mechanischen Eigenschaften von Vimentin Filamenten zu meiner Arbeit beigetragen.

Stefan Winheim bin ich dankbar für die ausführliche Anleitung für das anfärben von Vimentin mit maleimid Farbstoffen und einige schöne Tage in Heidelberg.

Susanne Bauch hat den Großteil der Vimentin Herstellung übernommen, für ihren Beitrag zu meinen Experimenten bin ich ihr sehr dankbar.

Wiebke Möbius hat mit der Aufnahme von Elektronenmikroskopiebildern von Vimentin Filamenten einen sehr geschätzten Beitrag zu unserer Qualitätskontrolle geleistet. 
Guobin Bao und Alexander Brinkmann haben mich bei meinem Einstieg in die Messungen an einem FCS freundlich angeleitet und viele nützliche Hinweise gegeben. Für ihre Hilfe bei der Konzeption der FCCS Apparatur in unserem Labor bin ich sehr dankbar.

Jochen Herbst und Jörg Malindretos möchte ich für ihre Unterstützung im Reinraum danken.

Meinen Kollegen und Büromitbewohnern möchte ich für die angenehme Atmosphäre im Institut und der Arbeitsgruppe danken. Insbesondere möchte ich mich bei Susanne Bauch, Martha Brennich, Christian Dammann, Jannick LangpfahlKlabes, Jens Nolting, Rabea Sandmann, Viktor Schroeder, Sarah Schwarz und Britta Weinhausen für die immer freundliche Hilfe bei kleinen und großen Fragen bedanken. Ohne euch wäre meine Zeit am Institut sicher nicht so schön gewesen.

Nicht zuletzt möchte ich Annika, meinen Eltern Hilmar und Ulrike sowie meinen Geschwistern für die aufmunternden Worte und den Rückhalt den ihr mir immer gegeben habt, danken. 